\title{
The singularities and birational geometry of the compactified universal Jacobian
}

\author{
Sebastian Casalaina-Martin, Jesse Leo Kass and Filippo Viviani
}

\begin{abstract}
In this paper, we establish that the singularities of the compactified universal Jacobian are canonical if the genus is at least four. As a corollary, we determine the Kodaira dimension and the Iitaka fibration of the compactified universal Jacobian for every degree and genus. We also determine the birational automorphism group for every degree if the genus is at least twelve. This extends work of G. Farkas and A. Verra, as well as that of G. Bini, C. Fontanari and the third author.
\end{abstract}

\section{Introduction}

Jacobians of non-singular curves are principally polarized abelian varieties, which from the perspective of birational geometry are among the simplest varieties. On the other hand, for a family of non-singular curves, the relative Jacobian may exhibit more interesting birational behavior, not necessarily reflective of the birational geometry of the base. For instance, over the moduli space of non-singular, genus $g \geqslant 2$, automorphism-free curves $M_{g}^{\circ}$, there is a universal curve $C_{g}^{\circ}$ and, consequently, a universal Jacobian $\operatorname{Pic}^{0}\left(C_{g}^{\circ} / M_{g}^{\circ}\right)$. In this paper, we investigate the birational geometry of this space and show, for instance, that the Kodaira dimension of $\operatorname{Pic}^{0}\left(C_{g}^{\circ} / M_{g}^{\circ}\right)$ can be different from the Kodaira dimension of $M_{g}^{\circ}$.

More generally, for any integer $d$, Caporaso [Cap94] (see also [Pan96]) has constructed a compactified universal Jacobian $\pi: \bar{J}_{d, g} \rightarrow \bar{M}_{g}$ over the moduli space of Deligne-Mumford stable curves, the fiber of which over a non-singular, automorphism-free curve $C$ is the degree $d$ Jacobian $J^{d} C$. In particular, $\bar{J}_{0, g}$ provides a compactification of the universal Jacobian. In this paper, we focus on two main problems concerning the birational geometry of these spaces, namely determining the Kodaira dimension and determining the birational automorphism group. These problems go back at least to Caporaso's work and have been investigated recently by Farkas and Verra [FV13] and Bini, Fontanari and the third author [BFV12] in special cases.

Received 6 November 2014, accepted in final form 9 August 2016.

2010 Mathematics Subject Classification 14D20, 14H40, 14E99, 14D15, 14H20.

Keywords: compactified universal Jacobian, stable curves, torsion-free sheaves, canonical singularities, toric singularities, Kodaira dimension, Iitaka fibration, birational automorphisms.

This journal is (C) Foundation Compositio Mathematica 2017. This article is distributed with Open Access under the terms of the Creative Commons Attribution Non-Commercial License, which permits non-commercial reuse, distribution, and reproduction in any medium, provided that the original work is properly cited. For commercial re-use, please contact the Foundation Compositio Mathematica.

The first author was supported by NSF grant DMS-1101333. The second author was supported by NSF grant DMS-0502170. The third author has been partially supported by the MIUR-FIRB project Spazi di moduli $e$ applicazioni and by the CMUC (Centre for Mathematics of the University of Coimbra) - UID/MAT/00324/2013, funded by the Portuguese Government through FCT/MEC and co-funded by the European Regional Development Fund through the Partnership Agreement PT2020. 


\section{S. Casalaina-Martin, J. L. Kass and F. Viviani}

Due to [BFV12], the main point needed to answer these questions in full generality is to provide a good description of the local structure of $\bar{J}_{d, g}$. In this paper, we investigate this question in detail, providing an explicit description of the complete local ring at a point as well as formulas for various invariants of the ring in terms of the dual graphs of the curves. In particular, we establish that $\bar{J}_{d, g}$ has canonical singularities.

Theorem A. Assume $\operatorname{char}(k)=0$. If $g \geqslant 4$, then the compactified universal Jacobian $\bar{J}_{d, g}$ has canonical singularities for any $d \in \mathbb{Z}$.

The arguments build on the previous work of the authors in two ways. First, extending the deformation theory in [CKV15], we are able to reduce the problem to the study of a special class of combinatorial rings, called cographic toric face rings, investigated in [CKV13]. In full generality, these rings can exhibit poor behavior (see [CKV13, §5.1]). However, as it turns out, the rings appearing from the deformation theory of the compactified universal Jacobian form a special class of rings with mild singularities. The specific cographic rings appearing in this paper will be denoted by $U(\Gamma)$ and are defined from the data of a graph $\Gamma$ (Definition 3.1). Our main result for these rings is the following theorem.

Theorem B. Let $\Gamma$ be a finite, connected graph, and let $k$ be an algebraically closed field. The cographic toric ring $U(\Gamma)$ is a finitely generated, integral $k$-algebra, and the singularities of the associated variety $\operatorname{Spec} U(\Gamma)$ are Gorenstein, rational, and terminal.

Using the results in [CKV13], together with standard results on toric varieties, we are also able to establish a number of further properties of the rings $U(\Gamma)$ (and consequently $\bar{J}_{d, g}$ ) in terms of invariants of the graph $\Gamma$, including the dimension (Corollary 5.2), the dimension of the tangent space (Proposition 5.8), and the multiplicity (Theorem 5.12).

From Theorem A and the work of Bini-Fontanari-Viviani, one obtains the following consequence for the birational geometry of $\bar{J}_{d, g}$.

Corollary C. Assume char $(k)=0$. The Kodaira dimension of the universal Jacobian $\bar{J}_{d, g}$ is given by

$$
\kappa\left(\bar{J}_{d, g}\right)= \begin{cases}-\infty & \text { if } g \leqslant 9 \\ 0 & \text { if } g=10 \\ 19 & \text { if } g=11 \\ 3 g-3 & \text { if } g \geqslant 12\end{cases}
$$

Moreover, for $g \geqslant 10$, the Iitaka fibration of $J_{d, g}$ is given as follows:

(i) For $g \geqslant 12$, the Iitaka fibration is the forgetful morphism $\pi: \bar{J}_{d, g} \rightarrow \bar{M}_{g}$.

(ii) For $g=11$, the Iitaka fibration is the rational map $\bar{J}_{d, 11} \rightarrow \mathcal{F}_{11}$, where $\mathcal{F}_{g}$ is the moduli space of $K 3$ surfaces with polarization of degree $2 g-2$ and the rational map takes a general pair $(C, L)$ to the pair $\left(S, \mathcal{O}_{S}(C)\right)$, where $S$ is the unique $K 3$ containing $C$ (see $[$ Muk96]).

(iii) For $g=10$, the Iitaka fibration is the structure morphism $\bar{J}_{d, 10} \rightarrow \operatorname{Spec} k$.

For $g=22$ and $g \geqslant 24$, the statement on the Kodaira dimension follows from general results in birational geometry, together with well-known results for $\bar{M}_{g}$ (see Remark 8.10). In the remaining range, the result was proven by Bini-Fontanari-Viviani [BFV12, Theorem 1.2] under the numerical condition that $\operatorname{gcd}(d+1-g, 2 g-2)=1$ or $g=23$, and by Farkas-Verra [FV13] 


\section{THE SINGULARITIES AND BIRATIONAL GEOMETRY}

in the special case $d=g$. In particular, the case $d=0$ was not known. We also point out that while we have obtained here a complete classification of the Kodaira dimension for the universal Jacobian, the Kodaira dimension of the moduli space of curves is still unknown in the range $17 \leqslant g \leqslant 21$ and for $g=23$. Finally, for $10 \leqslant g \leqslant 16$, we have $\kappa\left(\bar{J}_{d, g}\right) \neq \kappa\left(\bar{M}_{g}\right)$. We direct the reader to (8.35) for more details, as well as to Remark 8.12, which compares these numerics with the recent work of Farkas-Verra [Far10, FV12, FV14, Far12] on the moduli space of theta characteristics.

Another immediate observation is that the Kodaira dimension is independent of $d$. One might guess that the reason for this is that $\bar{J}_{d, g}$ is birational to $\bar{J}_{d^{\prime}, g}$ for different $d$ and $d^{\prime}$. Our next result shows that this is not generally the case.

Corollary D. Assume char $(k)=0$ and $g \geqslant 12$. If $\eta: J_{d, g} \rightarrow J_{d^{\prime}, g}$ is a birational map, then $d^{\prime}= \pm d+n(2 g-2)$ and $\eta$ is given by the map sending $(C, L) \in J_{d, g}$ into $\left(C, L^{ \pm 1} \otimes \omega_{C}^{n}\right) \in J_{d^{\prime}, g}$. In particular, the following hold:

(i) The universal Jacobian $J_{d, g}$ is birational to $J_{d^{\prime}, g}$ if and only if $d^{\prime} \equiv \pm d \bmod 2 g-2$.

(ii) The group $\operatorname{Bir}\left(J_{d, g}\right)$ of birational automorphisms of $J_{d, g}$ is given by

$$
\operatorname{Bir}\left(J_{d, g}\right)= \begin{cases}\mathbb{Z} / 2 \mathbb{Z} & \text { if } d=n(g-1) \text { for some } n \in \mathbb{Z}, \\ \{\mathrm{Id}\} & \text { otherwise. }\end{cases}
$$

Moreover, if $d=n(g-1)$ for some $n \in \mathbb{Z}$, then the generator of $\operatorname{Bir}\left(J_{d, g}\right)$ is the birational automorphism sending $(C, L)$ into $\left(C, L^{-1} \otimes \omega_{C}^{n}\right)$.

This was proven by Bini-Fontanari-Viviani [BFV12, Theorem 1.7] in the special case gcd $(d+$ $1-g, 2 g-2)=1($ or $g \geqslant 22)$ and builds on work of Caporaso [Cap94].

This paper is organized as follows. In Section 2, we review terminology concerning graphs and various constructions with graphs that will appear later. In Section 3, we define the combinatorial rings $U(\Gamma)$ and establish some first properties of the rings. In Section 4, we establish some specific presentations of the rings which are useful for later computations and also for connecting the rings with deformations. In Section 5, we discuss the singularities of the rings $U(\Gamma)$. In Section 6, we describe the rings as invariants for a group action, which provides the framework for the connection with deformations of sheaves. In Section 7, we provide some examples of these rings. In Section 8, we make the connection with the compactified universal Jacobian and establish the results on the singularities, Kodaira dimension, and birational automorphism group.

The paper ends with an appendix in which we investigate the singularities of finite quotients of toric varieties. More specifically, the focus is on establishing a Reid-Tai-Shepherd-Barron criterion for singular toric varieties, that is, a numerical condition that can be used to determine when a finite quotient of a singular toric variety has canonical or terminal singularities. The main result is Proposition A.6, which in conjunction with Theorem A.11 is a direct generalization of the Reid-Tai-Shepherd-Barron criterion. While we expect that the generalization is well known to the experts, we were not aware of a reference and include proofs here.

\section{Preliminaries on graphs}

In this section, we introduce some constructions on graphs that we will use in this paper. 


\section{S. Casalaina-Martin, J. L. Kass and F. Viviani}

\subsection{Graph notation}

Following Serre [Ser80, $\S 2.1$ ], a graph $\Gamma$ consists of the data

$$
(\vec{E} \underset{t}{\stackrel{s}{\rightleftarrows}} V, \vec{E} \stackrel{\iota}{\rightarrow} \vec{E})
$$

where $V$ and $\vec{E}$ are sets, $\iota$ is a fixed-point-free involution, and $s$ and $t$ are maps satisfying $s(\vec{e})=t(\iota(\vec{e}))$ for all $\vec{e} \in \vec{E}$. The maps $s$ and $t$ are called the source and target maps, respectively. We call $V=: V(\Gamma)$ the set of vertices. We call $\vec{E}=: \vec{E}(\Gamma)$ the set of oriented edges.

We define the set of (unoriented) edges to be $E(\Gamma)=E:=\vec{E} / \iota$. Given an oriented edge $\vec{e} \in \vec{E}$, we will denote by $\underline{\vec{e}}$ the class of $\vec{e}$ in $E$. An orientation of an edge $e \in E$ is a representative for $e$ in $\vec{E}$; we use the notation $\vec{e}$ and $\overleftarrow{e}$ for the two possible orientations of $e$. An orientation of a graph $\Gamma$ is a section $\phi: E \rightarrow \vec{E}$ of the quotient map. An oriented graph consists of a pair $(\Gamma, \phi)$, where $\Gamma$ is a graph and $\phi$ is an orientation. Given an oriented graph, we say that $\phi(e)$ is the positive orientation of the edge $e \in E$. Given a subset $S \subseteq E$, we define $\vec{S} \subseteq \vec{E}$ to be the set of all orientations of the edges in $S$.

We will say that two edges of a graph are parallel if they connect the same (not necessarily distinct) vertices. We say that an edge of a connected graph is a separating edge if removing the edge disconnects the graph. Two edges of a connected graph are a separating pair if they are both non-separating edges and if removing the two edges disconnects the graph.

If $\Gamma$ is connected, then we say that an orientation $\phi$ of $\Gamma$ is totally cyclic if there does not exist a proper non-empty subset $W \subset V(\Gamma)$ such that the edges between $W$ and its complement $V(\Gamma) \backslash W$ all go in the same direction (that is, either all these edges are oriented from $W$ to $V(\Gamma) \backslash W$ or all are oriented in the opposite direction). If $\Gamma$ is disconnected, then we say that an orientation of $\Gamma$ is totally cyclic if the orientation induced on each connected component of $\Gamma$ is totally cyclic.

A graph $\Gamma$ is called cyclic if it is connected, free from separating edges, and satisfies $b_{1}(\Gamma):=$ $|E(\Gamma)|-|V(\Gamma)|+1=1$. We will also call a cyclic graph a circuit. A cyclic graph together with a totally cyclic orientation is called an oriented circuit. A loop is a circuit with a single edge.

\subsection{Ordinary homology and oriented homology}

Given any graph $\Gamma$, we can form its ordinary homology (which coincides with the homology of the underlying topological space) and its oriented homology.

Let $\mathbb{C}_{0}(\Gamma, \mathbb{Z})$ be the free $\mathbb{Z}$-module with basis $V(\Gamma)$, let $\mathbb{C}_{1}(\Gamma, \mathbb{Z})$ be the free $\mathbb{Z}$-module generated by $\vec{E}(\Gamma)$, and consider the boundary map $\mathbb{D}$ defined as

$$
\begin{aligned}
\mathbb{D}: \mathbb{C}_{1}(\Gamma, \mathbb{Z}) & \rightarrow \mathbb{C}_{0}(\Gamma, \mathbb{Z}), \\
\vec{e} & \mapsto t(\vec{e})-s(\vec{e}) .
\end{aligned}
$$

We will denote by $\mathbb{H}_{\bullet}(\Gamma, \mathbb{Z})$ the groups obtained from the homology of $\mathbb{C} \bullet(\Gamma, \mathbb{Z})$, and we will call them the oriented homology groups of $\Gamma$. Let $\left(\right.$, ) be the unique scalar product on $\mathbb{C}_{1}(\Gamma, \mathbb{R})=$ $\mathbb{C}_{1}(\Gamma, \mathbb{Z}) \otimes_{\mathbb{Z}} \mathbb{R}$ (and also its restriction to $\mathbb{H}_{1}(\Gamma, \mathbb{Z})$ ) such that the elements of $\vec{E}(\Gamma)$ form an orthonormal basis.

Let $C_{0}(\Gamma, \mathbb{Z})=\mathbb{C}_{0}(\Gamma, \mathbb{Z})$, let $C_{1}(\Gamma, \mathbb{Z})$ be the quotient of $\mathbb{C}_{1}(\Gamma, \mathbb{Z})$ by the relation $\overleftarrow{e}=-\vec{e}$ for 
every $e \in E(\Gamma)$, and consider the boundary map

$$
\begin{aligned}
\partial: C_{1}(\Gamma, \mathbb{Z}) & \rightarrow C_{0}(\Gamma, \mathbb{Z}), \\
{[\vec{e}] } & \mapsto t(\vec{e})-s(\vec{e}),
\end{aligned}
$$

where we denote by $[\vec{e}]$ the class of $\vec{e}$ in $C_{1}(\Gamma, \mathbb{Z})$. We will denote by $H_{\bullet}(\Gamma, \mathbb{Z})$ the groups obtained from the homology of $C_{\bullet}(\Gamma, \mathbb{Z})$, and we will call them the ordinary homology groups of $\Gamma$. Note that $H_{\bullet}(\Gamma, \mathbb{Z})$ is isomorphic to the homology of the underlying topological space of $\Gamma$. Let $($, be the unique scalar product on $C_{1}(\Gamma, \mathbb{Z})$ (and also its restriction to $H_{1}(\Gamma, \mathbb{Z})$ ) such that

$$
\begin{aligned}
& ([\vec{e}],[\vec{e}])=-([\vec{e}],[\overleftarrow{e}])=1 \text { for any } e \in E \\
& \left(\left[\vec{e}_{1}\right],\left[\vec{e}_{2}\right]\right)=0 \text { for any } \vec{e}_{1}, \vec{e}_{2} \in \vec{E} \text { such that }\left[\vec{e}_{1}\right] \neq \pm\left[\vec{e}_{2}\right]
\end{aligned}
$$

For a connected graph $\Gamma$, the coranks of the images of $\mathbb{D}$ and $\partial$ are one. Consequently, for a connected graph, we have

$$
\begin{aligned}
& \operatorname{rank} H_{1}(\Gamma, \mathbb{Z})=|E(\Gamma)|-|V(\Gamma)|+1=: b_{1}(\Gamma), \\
& \operatorname{rank} \mathbb{H}_{1}(\Gamma, \mathbb{Z})=2|E(\Gamma)|-|V(\Gamma)|+1=b_{1}(\Gamma)+|E(\Gamma)| .
\end{aligned}
$$

In order to determine the relationship between ordinary and oriented homology, consider the following commutative diagram:

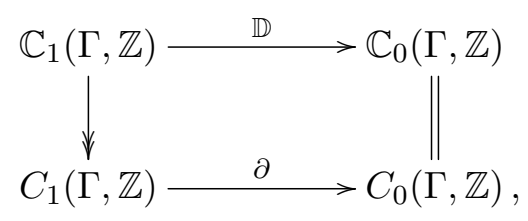

where the left vertical map send $\vec{e}$ into $[\vec{e}]$. This diagram $(2.4)$ induces an equality $\mathbb{H}_{0}(\Gamma, \mathbb{Z})=$ $H_{0}(\Gamma, \mathbb{Z})$ and a surjection $\mathbb{H}_{1}(\Gamma, \mathbb{Z}) \rightarrow H_{1}(\Gamma, \mathbb{Z})$, whose kernel can be described as follows.

LEMma 2.1. The kernel of the natural surjection $\mathbb{H}_{1}(\Gamma, \mathbb{Z}) \rightarrow H_{1}(\Gamma, \mathbb{Z})$ is generated by $\{\vec{e}+$ $\overleftarrow{e}\}_{e \in E(\Gamma)}$

Proof. From the definition of $\mathbb{D}$, we have $\vec{e}+\overleftarrow{e} \in \mathbb{H}_{1}(\Gamma, \mathbb{Z})$. Clearly, $\vec{e}+\overleftarrow{e}$ also maps to 0 in $C_{1}(\Gamma, \mathbb{Z})$. On the other hand, suppose that $\sum_{e \in E}\left(a_{e} \vec{e}+b_{e} \overleftarrow{e}\right) \in \mathbb{H}_{1}(\Gamma, \mathbb{Z})$ is in the kernel of the above map. Then, by definition, $\sum_{e \in E}\left(a_{e}-b_{e}\right)[\vec{e}]=0$, and so $a_{e}=b_{e}$ for all $e \in E$ since $\{[\vec{e}]\}$ is a basis for $C_{1}(\Gamma, \mathbb{Z})$.

\subsection{Doubled graphs and doubled orientations}

In this section, we introduce a class of graphs, called doubled graphs, together with canonical totally cyclic orientations of them, called doubled orientations, which are obtained from a graph by doubling its edges.

Definition 2.2. Let $\Gamma$ be a connected graph. Define the doubled graph of $\Gamma$, denoted $\Gamma^{d}$, to be the graph obtained by doubling the edges of $\Gamma$; that is, $\Gamma^{d}$ is the graph obtained from $\Gamma$ by replacing each edge $e$ of $\Gamma$ with a pair of parallel edges $e^{\prime}$ and $e^{\prime \prime}$ of $\Gamma^{d}$ having the same endpoints as $e$ (see Figure 1). To be precise, we have $V\left(\Gamma^{d}\right)=V(\Gamma)$ and $\vec{E}\left(\Gamma^{d}\right)=\bigcup_{\vec{e} \in \vec{E}}\left\{\vec{e}^{\prime}, \vec{e}^{\prime \prime}\right\}$, and we define

$$
s\left(\vec{e}^{\prime}\right)=s\left(\vec{e}^{\prime \prime}\right)=s(\vec{e}), \quad t\left(\vec{e}^{\prime}\right)=t\left(\vec{e}^{\prime \prime}\right)=t(\vec{e}) \quad \text { and } \quad \iota\left(\vec{e}^{\prime}\right)=\overleftarrow{e}^{\prime}, \iota\left(\vec{e}^{\prime \prime}\right)=\overleftarrow{e}^{\prime \prime}
$$


S. Casalaina-Martin, J. L. Kass and F. Viviani

Unoriented edges $E$

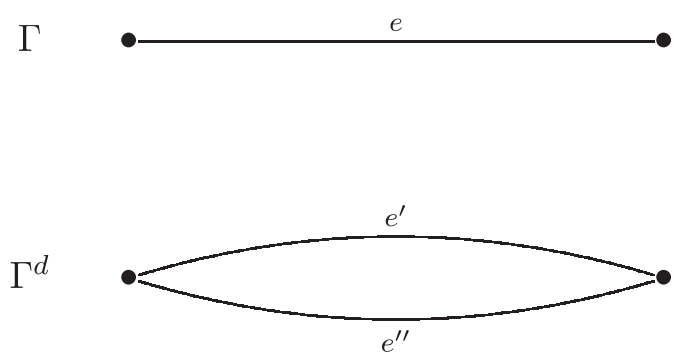

Oriented edges $\vec{E}$

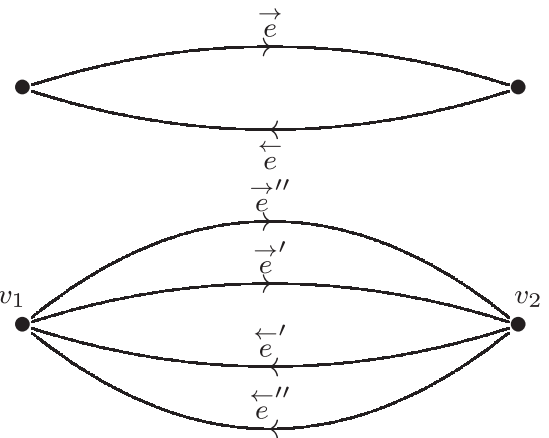

Figure 1. Doubled graph

Unoriented edges $E$

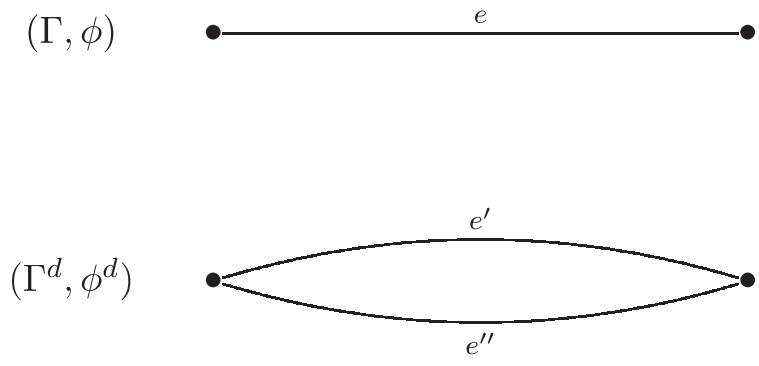

Oriented edges $\vec{E}$
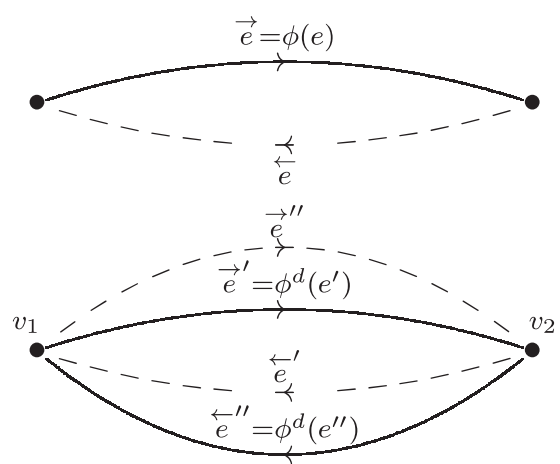

FIGURE 2. Doubled orientation

Note that

$$
E\left(\Gamma^{d}\right)=\bigcup_{e \in E(\Gamma)}\left\{e^{\prime}, e^{\prime \prime}\right\},
$$

where we use the convention that if $e=\underline{\vec{e}}$, then $e^{\prime}=\underline{\vec{e}}^{\prime}$ and $e^{\prime \prime}=\underline{\vec{e}}^{\prime \prime}$.

The graph $\Gamma^{d}$ drawn with its unoriented edges looks like the graph $\Gamma$ drawn with its oriented edges (see Figure 1). In this way, choosing an identification of edges gives an orientation $\phi^{d}$ of $\Gamma^{d}$. In fact, given an orientation $\phi$ of $\Gamma$, one obtains an orientation $\phi^{d}$ of $\Gamma^{d}$ by orienting each edge $e^{\prime}$ in the same direction as $\phi(e)$ and each edge $e^{\prime \prime}$ in the opposite direction (see Figure 2).

Definition 2.3. Given an orientation $\phi$ of $\Gamma$, define the doubled orientation

$$
\begin{aligned}
& \phi^{d}: E\left(\Gamma^{d}\right) \rightarrow \vec{E}\left(\Gamma^{d}\right), \\
& \phi^{d}\left(e^{\prime}\right)=\phi(e)^{\prime}, \quad \phi^{d}\left(e^{\prime \prime}\right)=\iota\left(\phi(e)^{\prime \prime}\right) .
\end{aligned}
$$

Lemma 2.4. The doubled orientation $\phi^{d}$ on $\Gamma^{d}$ is canonical; that is, it does not depend on the choice of $\phi$ up to automorphisms of $\Gamma^{d}$, and it is totally cyclic. 
Proof. Choose an (unoriented) edge $f \in E(\Gamma)$, define a new orientation $\phi^{f}$ of $\Gamma$ by reversing the orientation on $f$, that is, setting

$$
\phi^{f}(e)= \begin{cases}\iota(\phi(f)) & \text { if } e=f, \\ \phi(e) & \text { if } e \neq f .\end{cases}
$$

Define an automorphism $\psi$ of $\Gamma^{d}$ that is the identity on vertices, exchanges $f^{\prime}$ and $f^{\prime \prime}$, and fixes $e^{\prime}$ and $e^{\prime \prime}$ for all other edges $e \neq f$ of $\Gamma$. Then, clearly, $\psi$ will send the orientation $\phi^{d}$ into $\left(\phi^{f}\right)^{d}$. Since every other orientation of $\Gamma$ can be obtained from $\phi$ by iteratively applying the above construction, we have shown that $\phi^{d}$ is canonical.

The fact that $\phi^{d}$ is totally cyclic follows easily from the fact that any two parallel (unoriented) edges $e^{\prime}$ and $e^{\prime \prime}$ of $\Gamma^{d}$ associated with an edge $e$ of $\Gamma$ are given opposite orientations by $\phi^{d}$.

The oriented homology of $\Gamma$ is canonically isomorphic to the ordinary homology of $\Gamma^{d}$. In order to prove this, fix an orientation $\phi$ of $\Gamma$ and consider the diagram

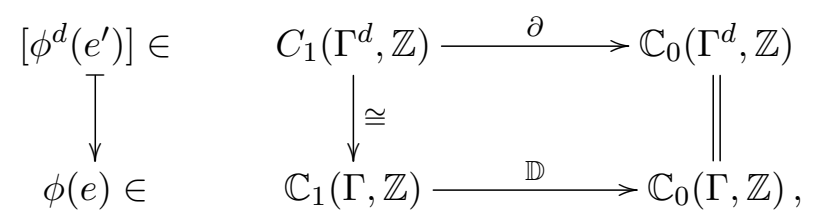

where the left vertical map is the group isomorphism obtained by, for each $e \in E(\Gamma)$, sending $\left[\phi^{d}\left(e^{\prime}\right)\right] \in C_{1}\left(\Gamma^{d}, \mathbb{Z}\right)$ into $\phi(e) \in \mathbb{C}_{1}(\Gamma, \mathbb{Z})$ (and $\left[\phi^{d}\left(e^{\prime \prime}\right)\right]$ to $\left.\iota \phi(e)\right)$. In short, if we choose a doubled orientation $\phi^{d}$ on $\Gamma^{d}$, then $C_{1}\left(\Gamma^{d}, \mathbb{Z}\right)$ can be given a basis consisting of the oriented edges determined by $\phi^{d}$; these edges are in bijection (including orientation) with the collection of all oriented edges of $\Gamma$, which form a basis of $\mathbb{C}_{1}(\Gamma, \mathbb{Z})$ (see Figure 2).

LEMma 2.5. The diagram (2.5) is commutative, and it induces an isomorphism $H_{i}\left(\Gamma^{d}, \mathbb{Z}\right) \cong$ $\mathbb{H}_{i}(\Gamma, \mathbb{Z})$ for $i=0,1$.

Proof. This is straightforward to check and is left to the reader.

\subsection{The affine semigroup $\operatorname{ring} R(\Gamma, \phi)$ and its associated toric variety $X_{(\Gamma, \phi)}$}

In this section, we review the definition of the ring $R(\Gamma, \phi)$ from $[\mathrm{CKV} 13, \S 4]$. Let $(\Gamma, \phi)$ be a graph with a totally cyclic orientation. Consider the pointed full-dimensional rational polyhedral cone

$$
\sigma_{\Gamma}(\phi):=\bigcap_{e \in E(\Gamma)}\{(\cdot, \phi(e)) \geqslant 0\} \subset H_{1}(\Gamma, \mathbb{Z}) \otimes_{\mathbb{Z}} \mathbb{R} .
$$

(This was denoted $\sigma(\emptyset, \phi)$ in [CKV13, §3].) According to Gordan's lemma (see, for example, [CLS11, Proposition 1.2.17]), the semigroup

$$
C_{\Gamma}(\phi):=\sigma_{\Gamma}(\phi) \cap H_{1}(\Gamma, \mathbb{Z}) \subset H_{1}(\Gamma, \mathbb{Z})=\mathbb{Z}^{b_{1}(\Gamma)}
$$

is a positive, normal, affine semigroup, that is, a finitely generated subgroup isomorphic to a subsemigroup of $\mathbb{Z}^{d}$ for some $d \in \mathbb{N}$, such that 0 is the unique invertible element and such that if $m \cdot z \in C_{\Gamma}(\phi)$ for some $m \in \mathbb{N}$ and $z \in \mathbb{Z}^{d}$, then $z \in C_{\Gamma}(\phi)$.

Recall [CKV13, Definition 4.2] that we define

$$
R(\Gamma, \phi):=k\left[C_{\Gamma}(\phi)\right]
$$




\section{S. Casalaina-Martin, J. L. Kass and F. Viviani}

to be the affine semigroup ring associated with $C_{\Gamma}(\phi)$, that is, the $k$-algebra whose underlying vector space has basis $\left\{X^{c}: c \in C_{\Gamma}(\phi)\right\}$ and whose multiplication is defined by $X^{c} \cdot X^{c^{\prime}}:=X^{c+c^{\prime}}$. The ring $R(\Gamma, \phi)$ is a normal, Cohen-Macaulay domain of dimension equal to (for example, [CKV13, Lemma 4.3])

$$
\operatorname{dim} R(\Gamma, \phi)=\operatorname{dim} \sigma_{\Gamma}(\phi)=b_{1}(\Gamma)
$$

The affine variety

$$
X_{(\Gamma, \phi)}:=\operatorname{Spec} R(\Gamma, \phi)
$$

is the toric variety associated with the fan $\Sigma_{(\Gamma, \phi)}$ consisting of the dual cone

$$
\sigma_{\Gamma}(\phi)^{\vee} \subset H_{1}(\Gamma, \mathbb{Z})^{\vee} \otimes_{\mathbb{Z}} \mathbb{R}
$$

together with all its faces.

\section{The cographic toric variety $X_{\Gamma}$ and the cographic toric ring $U(\Gamma)$}

Fix a graph $\Gamma$. Using the notation of Section 2.2, set $\mathbb{M}_{\Gamma}:=\mathbb{H}_{1}(\Gamma, \mathbb{Z})$ and $\mathbb{N}_{\Gamma}:=\mathbb{H}_{1}(\Gamma, \mathbb{Z})^{\vee}$. Consider the pointed rational polyhedral cone

$$
\sigma_{\Gamma}:=\bigcap_{\vec{e} \in \vec{E}}\{(, \vec{e}) \geqslant 0\} \subset \mathbb{M}_{\Gamma} \otimes_{\mathbb{Z}} \mathbb{R},
$$

and denote its dual cone by $\sigma_{\Gamma}^{\vee} \subset \mathbb{N}_{\Gamma} \otimes_{\mathbb{Z}} \mathbb{R}$. Again from Gordan's lemma, the semigroup

$$
C(\Gamma):=\mathbb{H}_{1}(\Gamma, \mathbb{Z}) \cap \sigma_{\Gamma}
$$

is a positive, normal, affine semigroup.

Definition 3.1. (i) The cographic toric ring $U(\Gamma)$ of $\Gamma$ (over a base field $k$ ) is the affine semigroup $k$-algebra associated with $C(\Gamma)$; that is,

$$
U(\Gamma):=k[C(\Gamma)] .
$$

Explicitly, $U(\Gamma)$ is the $k$-algebra whose underlying vector space has basis $\left\{X^{c}: c \in C(\Gamma)\right\}$ and whose multiplication is defined by $X^{c} \cdot X^{c^{\prime}}:=X^{c+c^{\prime}}$.

(ii) The cographic toric variety $X_{\Gamma}$ of $\Gamma$ (over a base field $k$ ) is the affine variety

$$
X_{\Gamma}:=\operatorname{Spec} U(\Gamma)=\operatorname{Spec} k[C(\Gamma)] .
$$

Observe that $X_{\Gamma}$ is the (normal) toric variety associated with the rational polyhedral fan $\Sigma_{\Gamma}$ in $\mathbb{N}_{\Gamma} \otimes_{\mathbb{Z}} \mathbb{R}$ formed by $\sigma_{\Gamma}^{\vee}$ and all its faces. We describe $\sigma_{\Gamma}^{\vee}$ in more detail in Section 5 .

Example 3.2. Let $L$ be the loop graph, that is, the graph with one vertex $v$ and one unoriented edge $e$ which is a loop around $v$. Then $\mathbb{C}_{1}(L, \mathbb{Z})$ is freely generated by $\vec{e}$ and $\overleftarrow{e}$ and the boundary map $\mathbb{D}$ is trivial; hence $\mathbb{H}_{1}(L, \mathbb{Z})=\mathbb{C}_{1}(L, \mathbb{Z})=\langle\vec{e}, \overleftarrow{e}\rangle$. The cone $\sigma_{L}$ of (3.1) is the first quadrant in $\mathbb{H}_{1}(L, \mathbb{Z}) \otimes_{\mathbb{Z}} \mathbb{R} \cong \mathbb{R}^{2}$, and the semigroup $C(L)$ of $(3.2)$ is isomorphic to $\mathbb{N}^{2}$, generated by $\vec{e}$ and $\overleftarrow{e}$. Therefore

$$
U(L)=k[C(L)]=k\left[X^{\vec{e}}, X^{\overleftarrow{e}}\right] \cong k[X, Y] \quad \text { and } \quad X_{L}=\operatorname{Spec} U(L)=\mathbb{A}_{k}^{2}
$$

The cographic toric ring $U(\Gamma)$ and the cographic toric variety $X_{\Gamma}$ also admit another presentation in terms of the affine semigroup algebra (and its corresponding affine toric variety) associated to the double graph $\Gamma^{d}$ with its double orientation $\Gamma^{d}$; see Sections 2.3 and 2.4. 


\section{THE SINGULARITIES AND BIRATIONAL GEOMETRY}

Proposition 3.3. There is an isomorphism of $k$-algebras

$$
U(\Gamma) \cong R\left(\Gamma^{d}, \phi^{d}\right)
$$

inducing the isomorphism $X_{\Gamma} \cong X_{\left(\Gamma^{d}, \phi^{d}\right)}$ of toric varieties.

Proof. Comparing (2.6) with (3.1), it is easily checked that the isomorphism $H_{1}\left(\Gamma^{d}, \mathbb{Z}\right) \stackrel{\cong}{\longrightarrow}$ $\mathbb{H}_{1}(\Gamma, \mathbb{Z})$ of Lemma 2.5 sends the cone $\sigma_{\Gamma^{d}}\left(\phi^{d}\right)$ isomorphically into the cone $\sigma_{\Gamma}$ and hence the semigroup $C_{\Gamma^{d}}\left(\phi^{d}\right)$ isomorphically onto the semigroup $C(\Gamma)$. By taking the associated semigroup algebras, we get the isomorphism $R\left(\Gamma^{d}, \phi^{d}\right) \cong U(\Gamma)$ and, by passing to prime spectra, we obtain that $X_{\left(\Gamma^{d}, \phi^{d}\right)} \cong X_{\Gamma}$.

\section{An explicit presentation of the cographic toric ring $U(\Gamma)$}

The aim of this section is to give an explicit presentation of the cographic toric $\operatorname{ring} U(\Gamma)$, which also shows that $U(\Gamma)$ is a deformation of the cographic toric face ring $R(\Gamma)$ introduced and studied in [CKV13].

To begin, we define a map

$$
\psi: H_{1}(\Gamma, \mathbb{Z}) \times H_{1}(\Gamma, \mathbb{Z}) \rightarrow \mathbb{Z}_{\geqslant 0}^{E(\Gamma)} .
$$

For a cycle $z \in H_{1}(\Gamma, \mathbb{Z}) \subseteq C_{1}(\Gamma, \mathbb{Z})$, denote by $\operatorname{Supp}(z)$ (the support of $z$ ) the set of edges of $E(\Gamma)$ that appear with non-zero coefficient in $z$. Then we can write $z$ uniquely as

$$
z=\sum_{e \in \operatorname{Supp}(z)} a_{e}[\vec{e}]
$$

with $a_{e}>0$ for all $e \in \operatorname{Supp}(z)$.

Now if

$$
z^{(1)}=\sum_{e \in \operatorname{Supp}\left(z^{(1)}\right)} a_{e}^{(1)}\left[\vec{e}^{(1)}\right] \quad \text { and } \quad z^{(2)}=\sum_{e \in \operatorname{Supp}\left(z^{(2)}\right)} a_{e}^{(2)}\left[\vec{e}^{(2)}\right]
$$

then define

$$
\psi\left(z^{(1)}, z^{(2)}\right)_{e}:= \begin{cases}0 & \text { if } e \notin \operatorname{Supp}\left(z^{(1)}\right) \cap \operatorname{Supp}\left(z^{(2)}\right), \\ 0 & \text { if }\left[\vec{e}^{(1)}\right]=\left[\vec{e}^{(2)}\right], \\ \min \left(a_{e}^{(1)}, a_{e}^{(2)}\right) & \text { if }\left[\vec{e}^{(1)}\right]=-\left[\vec{e}^{(2)}\right] .\end{cases}
$$

Remark 4.1. While the definition above is made independent of an orientation and will be useful for the proof of the theorem below, the definition may be more transparent with the introduction of an orientation. So, for the sake of exposition, choose an orientation $\phi$ of $\Gamma$. Then a cycle $z \in H_{1}(\Gamma, \mathbb{Z})$ has a unique expression of the form $z=\sum_{e \in E} \alpha_{e} \phi(e)$, with the $\alpha_{e} \in \mathbb{Z}$. Now if $z^{(1)}=\sum_{e \in E} \alpha_{e}^{(1)} \phi(e)$ and $z^{(2)}=\sum_{e \in E} \alpha_{e}^{(2)} \phi(e)$, then define

$$
\psi\left(z^{(1)}, z^{(2)}\right)_{e}= \begin{cases}0 & \text { if } \alpha_{e}^{(1)} \alpha_{e}^{(2)} \geqslant 0 \\ \min \left(\left|\alpha_{e}^{(1)}\right|,\left|\alpha_{e}^{(2)}\right|\right) & \text { otherwise }\end{cases}
$$

In other words, we are just tallying the number of edges (with multiplicity) that two cycles share in opposite directions. Note that this definition agrees with the one above and does not depend on the choice of $\phi$. 


\section{S. Casalaina-Martin, J. L. Kass and F. Viviani}

Remark 4.2. It follows from [CKV13, Corollary 3.4] that $\psi\left(z^{(1)}, z^{(2)}\right)=0$ if and only if $z^{(1)}$ and $z^{(2)}$ lie in a common cone of the cographic fan $\mathcal{F}_{\Gamma}^{\perp}$ on $H_{1}(\Gamma, \mathbb{Z}) \otimes \mathbb{R}$ (see [CKV13, $\left.\S 1.4\right]$ and the references therein).

The key to obtaining an explicit presentation of the cographic toric ring $U(\Gamma)$ is the following alternative description of the semigroup $C(\Gamma)$ of $(3.2)$.

Proposition 4.3. The semigroup $C(\Gamma)$ is isomorphic to the set $H_{1}(\Gamma, \mathbb{Z}) \times \mathbb{Z}_{\geqslant 0}^{E(\Gamma)}$ endowed with the structure of semigroup given by

$$
\left(z_{1}, n_{1}\right) \times\left(z_{2}, n_{2}\right) \mapsto\left(z_{1}+z_{2}, \psi\left(z_{1}, z_{2}\right)+n_{1}+n_{2}\right) .
$$

In order to prove this proposition, we will need the following two lemmas.

LEMma 4.4. Under the natural surjection $\mathbb{H}_{1}(\Gamma, \mathbb{Z}) \rightarrow H_{1}(\Gamma, \mathbb{Z})$ induced by $(2.4)$, the semigroup $C(\Gamma)$ maps surjectively onto $H_{1}(\Gamma, \mathbb{Z})$.

Proof. We will prove this by constructing a section

$$
H_{1}(\Gamma, \mathbb{Z}) \rightarrow C(\Gamma) .
$$

Any cycle $z \in H_{1}(\Gamma, \mathbb{Z})$ can be written uniquely in the form $z=\sum_{e \in \operatorname{Supp}(z)} a_{e}[\vec{e}]$ with $a_{e}>0$. Thus

$$
z \mapsto \sum_{e \in \operatorname{Supp}(z)} a_{e} \vec{e}
$$

gives a well-defined map $H_{1}(\Gamma, \mathbb{Z}) \rightarrow C(\Gamma)$. It is clearly a section.

Lemma 4.5. The semigroup $C(\Gamma)$ is the sub-semigroup of $\mathbb{H}_{1}(\Gamma, \mathbb{Z})$ generated by $\{\vec{e}+\overleftarrow{e}\}_{e \in E(\Gamma)}$ and the image of the section $H_{1}(\Gamma, \mathbb{Z}) \rightarrow C(\Gamma)$ defined in (4.3) above.

Proof. Clearly, both $\{\vec{e}+\overleftarrow{e}\}_{e \in E(\Gamma)}$ as well as the image of the section $H_{1}(\Gamma, \mathbb{Z}) \rightarrow C(\Gamma)$ lie in $C(\Gamma)$.

Now, let $z \in C(\Gamma)$. Recall that by definition this means that $z=\sum_{\vec{e} \in \vec{E}} a_{\vec{e}} \vec{e}$ with $a_{\vec{e}} \geqslant 0$ for all $\vec{e} \in \vec{E}$. Let $z^{\prime}$ be the image of $z$ in $H_{1}(\Gamma, \mathbb{Z})$, and let $z^{\prime \prime}$ be the image of $z^{\prime}$ in $C(\Gamma)$ under the section. Then $z-z^{\prime \prime} \in \operatorname{ker}\left(\mathbb{H}_{1}(\Gamma, \mathbb{Z}) \rightarrow H_{1}(\Gamma, \mathbb{Z})\right)$. Thus, using Lemma 2.1, we can write $z=z^{\prime \prime}+\sum_{e \in E} b_{e}(\vec{e}+\overleftarrow{e})$. But, by the construction of $z^{\prime \prime}$, for all $e \in E$, the coefficient of either $\vec{e}$ or $\overleftarrow{e}$ in $z^{\prime \prime}$ is zero. Thus $b_{e} \geqslant 0$ for all $e \in E$, and we are done.

Proof of Proposition 4.3. It follows from Lemma 4.5 that there is an explicit bijection between the sets $C(\Gamma)$ and $H_{1}(\Gamma, \mathbb{Z}) \times \mathbb{Z}_{\geqslant 0}^{E(\Gamma)}$. By tracing the semigroup law on $C(\Gamma)$ via this bijection, we end up exactly with the semigroup law on $H_{1}(\Gamma, \mathbb{Z}) \times \mathbb{Z}_{\geqslant 0}^{E(\Gamma)}$ given by (4.2), and we are done.

From the explicit description of the semigroup $C(\Gamma)$ given in Proposition 4.3, we derive the following explicit presentation of the cographic toric ring $U(\Gamma)$.

Theorem 4.6. Let $\Gamma$ be a connected graph. Consider the $k$-algebra $D(\Gamma)$ whose underlying vector space has basis $\left\{X^{z} T^{\epsilon}: z \in H_{1}(\Gamma, \mathbb{Z}), \epsilon \in \mathbb{Z}_{\geqslant 0}^{E(\Gamma)}\right\}$ and whose multiplication is defined by the rule $X^{z} T^{\epsilon} \cdot X^{z^{\prime}} T^{\epsilon^{\prime}}:=X^{z+z^{\prime}} T^{\psi\left(z, z^{\prime}\right)+\epsilon+\epsilon^{\prime}}$. In other words,

$$
D(\Gamma):=\frac{k\left[X^{z}\right]_{z \in H_{1}(\Gamma, \mathbb{Z})}\left[T_{e}\right]_{e \in E}}{\left(X^{z} X^{z^{\prime}}-X^{z+z^{\prime}} \vec{T} \psi\left(z, z^{\prime}\right)\right)} .
$$

Then we have an isomorphism $U(\Gamma) \cong D(\Gamma)$. 


\section{THE SINGULARITIES AND BIRATIONAL GEOMETRY}

Proof. Observe that $D(\Gamma)$ is the semigroup $k$-algebra associated with the set $H_{1}(\Gamma, \mathbb{Z}) \times \mathbb{Z}_{\geqslant 0}^{E(\Gamma)}$ endowed with semigroup law (4.2). Then the result follows from Proposition 4.3.

Remark 4.7. From Theorem 4.6 together with Remark 4.2, it follows that by setting the variables $T_{e}$ equal to 0 , we get a surjective morphism of $k$-algebras

$$
U(\Gamma) \cong D(\Gamma) \rightarrow R(\Gamma),
$$

where $R(\Gamma)$ is the cographic toric face ring introduced in [CKV13, Definition 1.2]. Thus, the cographic toric variety $X_{\Gamma}$ can be viewed as a deformation of the cographic toric face variety $\operatorname{Spec} R(\Gamma)$ [CKV13, Definition 1.2] over the base Spec $k\left[T_{e}\right]_{e \in E}$.

\section{Singularities of $X_{\Gamma}$}

The aim of this section is to study the singularities of the cographic toric variety $X_{\Gamma}$. Recall from Definition 3.1 that $X_{\Gamma}$ is the (normal) toric variety associated with the rational polyhedral fan $\Sigma_{\Gamma}$ in $\mathbb{N}_{\Gamma} \otimes_{\mathbb{Z}} \mathbb{R}$ formed by the rational polyhedral cone $\sigma_{\Gamma}^{\vee}(3.1)$ and all its faces. The following lemma summarizes the basic properties of the cone $\sigma_{\Gamma}^{\vee}$.

Lemma 5.1. Let $\Gamma$ be a connected graph. Set $\mathbb{M}_{\Gamma}=\mathbb{H}_{1}(\Gamma, \mathbb{Z})$.

(i) The cone $\sigma_{\Gamma}^{\vee} \subseteq \mathbb{M}_{\Gamma}^{\vee}=\mathbb{N}_{\Gamma}$ is equal to

$$
\sigma_{\Gamma}^{\vee}=\left\{\sum_{\vec{e} \in \vec{E}(\Gamma)} a_{e} \cdot(, \vec{e}): a_{e} \geqslant 0\right\},
$$

where $(, \vec{e})$ denotes the element of $\mathbb{N}_{\Gamma}$ obtained by pairing an element of $\mathbb{M}_{\Gamma}$ with $\vec{e}$ via the scalar product $($, ) defined in Section 2.2.

(ii) The cone $\sigma_{\Gamma}^{\vee}$ is pointed and of full dimension in $\mathbb{N}_{\Gamma} \otimes \mathbb{R}$.

(iii) The extremal rays of $\sigma_{\Gamma}^{\vee}$ are of the form $\langle(, \vec{e})\rangle:=\mathbb{R}_{\geqslant 0} \cdot(, \vec{e})$ as $\vec{e}$ varies in $\vec{E}(\Gamma)$. Moreover, given $\vec{e}_{1} \neq \vec{e}_{2}$, we have

$$
\left\langle\left(, \vec{e}_{1}\right)\right\rangle=\left\langle\left(, \vec{e}_{2}\right)\right\rangle \quad \Longleftrightarrow \quad \underline{\vec{e}}_{1}=\underline{\vec{e}}_{2} \text { is a separating edge of } \Gamma .
$$

(iv) For every $\vec{e} \in \vec{E}(\Gamma)$, the primitive element of the ray $\langle(, \vec{e})\rangle$ with respect to the lattice $\mathbb{N}_{\Gamma}$ is $(, \vec{e})$, that is, $\langle(, \vec{e})\rangle \cap \mathbb{N}_{\Gamma}=\mathbb{Z}_{\geqslant 0} \cdot(, \vec{e})$.

Proof. Property (i) follows from (3.1), using the definition of a dual cone. Also, the first part of property (iii) follows from property (ii). We will deduce the remaining properties of $\sigma_{\Gamma}^{\vee}$ from the properties of its dual cone $\sigma_{\Gamma} \subset \mathbb{M}_{\Gamma}$, which is isomorphic to the cone $\sigma_{\Gamma^{d}}\left(\sigma^{d}\right) \subset H_{1}\left(\Gamma^{d}, \mathbb{Z}\right)$, as shown in the proof of Proposition 3.3.

According to [CKV13, Proposition 3.1], the cone $\sigma_{\Gamma^{d}}\left(\phi^{d}\right)$ is a pointed and full-dimensional cone in $H_{1}\left(\Gamma^{d}, \mathbb{Z}\right) \otimes \mathbb{R}$. By duality, we deduce that statement (ii) holds. 


\section{S. Casalaina-Martin, J. L. Kass and F. Viviani}

Now, observe that if $e$ is a non-separating edge of $\Gamma$, then the orientations $\phi_{\mid \Gamma^{d} \backslash\left\{e^{\prime}\right\}}^{d}$ and $\phi_{\mid \Gamma^{d} \backslash\left\{e^{\prime \prime}\right\}}^{d}$ induced by $\phi^{d}$ on the graphs $\Gamma^{d} \backslash\left\{e^{\prime}\right\}$ and $\Gamma^{d} \backslash\left\{e^{\prime \prime}\right\}$, respectively, are still totally cyclic. On the other hand, if $e$ is a separating edge of $\Gamma$, then the corresponding edges $e^{\prime}$ and $e^{\prime \prime}$ of $\Gamma^{d}$ form a pair of parallel edges; hence the orientation $\phi_{\mid \Gamma^{d} \backslash\left\{e^{\prime}, e^{\prime \prime}\right\}}$ induced by $\phi^{d}$ on $\Gamma^{d} \backslash\left\{e^{\prime}, e^{\prime \prime}\right\}$ is still totally cyclic, while neither the orientation induced by $\phi^{d}$ on $\Gamma^{d} \backslash\left\{e^{\prime}\right\}$ nor the one induced on $\Gamma^{d} \backslash\left\{e^{\prime \prime}\right\}$ is totally cyclic. Therefore, [CKV13, Proposition 3.1] implies that the codimension one faces of $\sigma_{\Gamma^{d}}\left(\phi^{d}\right)$ are given by (with the notation of Section 2.4)

(a) $\sigma_{\Gamma^{d} \backslash\left\{e^{\prime}\right\}}\left(\phi_{\mid \Gamma^{d} \backslash\left\{e^{\prime}\right\}}^{d}\right)$ and $\sigma_{\Gamma^{d} \backslash\left\{e^{\left.e^{\prime \prime}\right\}}\right.}\left(\phi_{\mid \Gamma^{d} \backslash\left\{e^{\prime \prime}\right\}}^{d}\right)$ for any non-separating edge $e$ of $\Gamma$,

(b) $\sigma_{\Gamma^{d} \backslash\left\{e^{\prime}, e^{\prime \prime}\right\}}\left(\phi_{\mid \Gamma^{d} \backslash\left\{e^{\prime}, e^{\prime \prime}\right\}}\right)$ for any separating edge $e$ of $\Gamma$.

The faces of type (a) are given by intersecting $\sigma_{\Gamma^{d}}\left(\phi^{d}\right)$ with, respectively, the hyperplanes $\left\{\left(\cdot, \phi^{d}\left(e^{\prime}\right)\right)=0\right\}$ and $\left\{\left(\cdot, \phi^{d}\left(e^{\prime \prime}\right)\right)=0\right\}$ for any non-separating edge $e$ of $\Gamma$. On the other hand, the faces of type (b) are given by intersecting with the hyperplanes $\left\{\left(\cdot, \phi^{d}\left(e^{\prime}\right)\right)=0\right\}=\left\{\left(\cdot, \phi^{d}\left(e^{\prime \prime}\right)\right)=\right.$ $0\}$ for any separating edge $e$ of $\Gamma$. By duality, we obtain property (iii).

For part (iv), consider the element $\vec{e}+\overleftarrow{e} \in \mathbb{H}_{1}(\Gamma, \mathbb{Z})=\mathbb{M}_{\Gamma}$. Since $(\vec{e}+\overleftarrow{e}, \vec{e})=1$, we get that $(, \vec{e})$ is the primitive element of the ray $\langle(, \vec{e})\rangle$.

Corollary 5.2. The variety $X_{\Gamma}$ does not contain torus factors and has dimension equal to

$$
\operatorname{dim} X_{\Gamma}=\operatorname{dim} \sigma_{\Gamma}^{\vee}=\operatorname{rank} \mathbb{H}_{1}(\Gamma, \mathbb{Z})=b_{1}(\Gamma)+|E(\Gamma)| .
$$

Proof. This follows directly from Lemma 5.1(ii), using [CLS11, Proposition 3.3.9(c)] and [BH98, Proposition 6.6.1].

We will want the following result describing the behavior of the cographic toric variety in the presence of separating edges and loops.

Lemma 5.3. Let $\Gamma$ be a connected graph with $n$ separating edges and $m$ loops, and let $\Gamma^{\prime}$ be the graph obtained from $\Gamma$ by contracting the separating edges and deleting the loops. Then we have

$$
X_{\Gamma}=\mathbb{A}_{k}^{n+2 m} \times X_{\Gamma^{\prime}} .
$$

Proof. Let $\left\{f_{1}, \ldots, f_{n}\right\}$ be the separating edges of $\Gamma$, let $\left\{e_{1}, \ldots, e_{m}\right\}$ be the loops of $\Gamma$, and set $\gamma_{i}:=\left[\vec{e}_{i}\right] \in H_{1}(\Gamma, \mathbb{Z})$. Clearly, we have $H_{1}(\Gamma, \mathbb{Z})=H_{1}\left(\Gamma^{\prime}, \mathbb{Z}\right) \oplus \bigoplus_{i=1}^{m}\left\langle\gamma_{i}\right\rangle$. Moreover, if we denote by $\psi$ the map (4.1) associated with $H_{1}(\Gamma, \mathbb{Z})$ and by $\psi^{\prime}$ the analogous map associated with $H_{1}\left(\Gamma^{\prime}, \mathbb{Z}\right)$, then we have

$$
\psi\left(z^{(1)}+\sum_{i} n_{i}^{(1)} \gamma_{i}, z^{(2)}+\sum_{i} n_{i}^{(2)} \gamma_{i}\right)_{e}= \begin{cases}\psi^{\prime}\left(z^{(1)}, z^{(2)}\right) & \text { if } e \notin\left\{e_{1}, \ldots, e_{m}\right\} \\ 0 & \text { if } e=e_{i} \text { and } n_{i}^{(1)} n_{i}^{(2)} \geqslant 0 \\ \min \left(\left|n_{i}^{(1)}\right|,\left|n_{i}^{(2)}\right|\right) & \text { if } e=e_{i} \text { and } n_{i}^{(1)} n_{i}^{(2)}<0\end{cases}
$$

for any $z^{(j)} \in H_{1}\left(\Gamma^{\prime}, \mathbb{Z}\right)$ and $n_{j}^{(i)} \in \mathbb{Z}$. This implies easily that (using the notation of Theorem 4.6)

$$
D(\Gamma)=D\left(\Gamma^{\prime}\right) \otimes_{k} \frac{k\left[X^{\gamma_{1}}, X^{-\gamma_{1}}, \ldots, X^{\gamma_{m}}, X^{-\gamma_{m}}, T_{e_{1}}, \ldots, T_{e_{m}}\right]}{\left(X^{\gamma_{1}} X^{-\gamma_{1}}-T_{e_{1}}, \ldots, X^{\gamma_{m}} X^{-\gamma_{m}}-T_{e_{m}}\right)} \otimes_{k} k\left[T_{f_{1}}, \ldots, T_{f_{n}}\right] .
$$

By passing to the prime spectra and using Theorem 4.6, we conclude.

Remark 5.4. A lengthier, but more elementary argument can be made for Lemma 5.3 directly from the definitions, without using Theorem 4.6. 


\section{THE SINGULARITIES AND BIRATIONAL GEOMETRY}

From the point of view of birational geometry, the singularities of $X_{\Gamma}$ are particularly nice.

Theorem 5.5. The variety $X_{\Gamma}$ is Gorenstein and terminal and has rational singularities.

Proof. It is well known that any (normal) toric variety has rational singularities (for example, [CLS11, Theorem 11.4.2]) and is Cohen-Macaulay (for example, [CLS11, Theorem 9.2.9]).

According to [CLS11, Proposition 8.2.12] (see also Proposition A.3), $X_{\Gamma}$ is Gorenstein, that is, the canonical divisor $K_{X_{\Gamma}}$ is Cartier, if and only if there exists an element $m \in \mathbb{M}_{\Gamma}$ such that $\left\langle m, u_{\rho}\right\rangle=1$ for any extremal ray $\rho$ of $\sigma_{\Gamma}^{\vee}$, where $\langle$,$\rangle denotes the canonical pairing between \mathbb{M}_{\Gamma}$ and $\mathbb{N}_{\Gamma}=\mathbb{M}_{\Gamma}^{\vee}$ and $u_{\rho}$ denotes the minimal generator of $\rho \cap \mathbb{N}_{\Gamma}$. Now, consider the following element of $\mathbb{C}_{1}(\Gamma, \mathbb{Z})$ :

$$
m_{\Gamma}:=\sum_{\vec{e} \in \vec{E}} \vec{e}=\sum_{e \in E}(\vec{e}+\overleftarrow{e})
$$

Since $\mathbb{D}(\overleftarrow{e})=-\mathbb{D}(\vec{e})$, we get $\mathbb{D}\left(m_{\Gamma}\right)=0$, and hence $m_{\Gamma} \in \mathbb{M}_{\Gamma}=\mathbb{H}_{1}(\Gamma, \mathbb{Z})$. By definition of the scalar product (see Section 2.2 ), we easily get

$$
\left(m_{\Gamma}, \vec{e}\right)=1 \text { for any } \vec{e} \in \vec{E} .
$$

For brevity, we will use the notation $u_{\vec{e}}$ for the element $(, \vec{e}) \in \mathbb{N}_{\Gamma}$ determined by $\vec{e} \in \vec{E}$. The above equality translates into

$$
\left\langle m_{\Gamma}, u_{\vec{e}}\right\rangle=1 .
$$

By Lemma 5.1, the rays of $\sigma_{\Gamma}^{\vee}$ are all of the form $\left\langle u_{\vec{e}}\right\rangle=\mathbb{R}_{\geqslant 0} \cdot u_{\vec{e}}\left(\right.$ as $\vec{e}$ varies in $\vec{E}$ ) and $u_{\vec{e}}$ is the primitive element of the ray $\left\langle u_{\vec{e}}\right\rangle$. Therefore, we conclude that $X_{\Gamma}$ is Gorenstein.

Finally, let us show that $X_{\Gamma}$ has terminal singularities. Since we have already proved that $X_{\Gamma}$ is Gorenstein, we conclude that $X_{\Gamma}$ has canonical singularities by [CLS11, Proposition 11.4.11]. Thus, using [CLS11, Proposition 11.4.12] (see also Proposition A.5), we conclude that in order to prove that $X_{\Gamma}$ has terminal singularities, it is (necessary and) sufficient to prove the following.

Claim 5.6. If $x \in \sigma_{\Gamma}^{\vee} \cap \mathbb{N}_{\Gamma}$ is such that $\left\langle m_{\Gamma}, x\right\rangle=1$, then $x=u_{\vec{e}}$ for some $\vec{e} \in \vec{E}$.

By Lemma 5.1(i), we can write $x=\sum_{\vec{e} \in \vec{E}} a_{\vec{e}} \cdot u_{\vec{e}}$ for certain $a_{\vec{e}} \in \mathbb{R}_{\geqslant 0}$. Note that such a representation may not be unique if the cone $\sigma_{\Gamma}^{\vee}$ is not simplicial, but we fix one such representation. By hypothesis, and recalling the definition of $m_{\Gamma}(5.2)$, we have

$$
1=\left\langle m_{\Gamma}, x\right\rangle=\left\langle\sum_{\overrightarrow{\vec{e}^{\prime} \in \vec{E}}} \vec{e}^{\prime}, \sum_{\vec{e} \in \vec{E}} a_{\vec{e}} \cdot u_{\vec{e}}\right\rangle=\sum_{\vec{e} \in \vec{E}} a_{\vec{e}} .
$$

Now, consider, for any $e \in E(\Gamma)$, the element $\gamma_{e}:=\vec{e}+\overleftarrow{e} \in \mathbb{C}_{1}(\Gamma, \mathbb{Z})$. As above, since $\mathbb{D}(\overleftarrow{e})=$ $-\mathbb{D}(\vec{e})$, we get $\mathbb{D}\left(\gamma_{e}\right)=0$, that is, $\gamma_{e} \in \mathbb{M}_{\Gamma}=\mathbb{H}_{1}(\Gamma, \mathbb{Z})$. Using (5.4) and the fact that $a_{\vec{e}} \geqslant 0$, we get

$$
\left\langle\gamma_{e}, x\right\rangle=a_{\vec{e}}+a_{\overleftarrow{e}} \in[0,1]
$$

Moreover, since $x \in \mathbb{N}_{\Gamma}$ and $\gamma_{e} \in \mathbb{M}_{\Gamma}$, we get $\left\langle\gamma_{e}, x\right\rangle \in \mathbb{Z}$; hence $\left\langle\gamma_{e}, x\right\rangle$ is equal either to 1 or to 0 . In the first case, all the coefficients $a_{\vec{e}}$ with $\vec{e} \neq \vec{e}$ or $\overleftarrow{e}$ must vanishes because of (5.4); hence $x=a_{\vec{e}} u_{\vec{e}}+a_{\overleftarrow{e}} u_{\leftarrow}$. In the second case, that is, if $\left\langle\gamma_{e}, x\right\rangle=0$, then necessarily $a_{\vec{e}}=a_{\overleftarrow{e}}=0$. We can therefore iterate the argument using all the edges of $\Gamma$ and, since $x \neq 0$, in the end we find 


\section{S. Casalaina-Martin, J. L. Kass and F. Viviani}

that necessarily

$$
x=a_{\vec{e}} u_{\vec{e}}+a_{\overleftarrow{e}} u_{\overleftarrow{e}} \quad \text { for some } e \in E(\Gamma)
$$

By virtue of Lemma 5.3, we may assume that $\Gamma$ does not have separating edges, so in particular $e$ is not a separating edge of $\Gamma$. Using this, it is easy to see that there exists a cycle $\gamma \in \mathbb{H}_{1}(\Gamma, \mathbb{Z})$ that contains $\vec{e}$ but not $\overleftarrow{e}$. Therefore, from (5.4) and (5.5), we get

$$
\langle\gamma, x\rangle=a_{\vec{e}} \in[0,1] .
$$

However, since $x \in \mathbb{N}_{\Gamma}$ and $\gamma \in \mathbb{M}_{\Gamma}$, we get $\langle\gamma, x\rangle \in \mathbb{Z}$; hence $a_{\vec{e}}=\langle\gamma, x\rangle$ is equal either to 1 or to 0 , which implies that $x$ is equal either to $u_{\vec{e}}$ or to $u_{\leftarrow}^{\leftarrow}$. Claim 5.6 is now proved.

We can now give a complete classification of the graphs $\Gamma$ for which $X_{\Gamma}$ is smooth or has finite quotient singularities.

Proposition 5.7. Let $\Gamma$ be a connected graph. The following conditions are equivalent:

(i) $X_{\Gamma}=\mathbb{A}_{k}^{b_{1}(\Gamma)+|E(\Gamma)|}$.

(ii) The variety $X_{\Gamma}$ is smooth.

(iii) The variety $X_{\Gamma}$ has finite quotient singularities.

(iv) The graph $\Gamma$ is tree-like; that is, $\Gamma$ becomes a tree after removing all the loops.

Proof. The implication (iv) $\Rightarrow$ (i) follows from Lemma 5.3.

The implications (i) $\Rightarrow$ (ii) $\Rightarrow$ (iii) are obvious.

(iii) $\Leftrightarrow$ (iv): First of all, from Lemma 5.3 we get that it is enough to prove the statement under the hypothesis that $\Gamma$ has no separating edges. Note that, under this assumption, condition (iv) now simply becomes that $\Gamma$ has a unique vertex. According to [CLS11, Theorem 11.4.8], the variety $X_{\Gamma}$ has finite quotient singularities if and only if $\sigma_{\Gamma}^{\vee}$ is simplicial, that is, its number of extremal rays is equal to its dimension. By Lemma 5.1(ii), the dimension of $\sigma_{\Gamma}^{\vee}$ is equal to

$$
\operatorname{dim} \sigma_{\Gamma}^{\vee}=\operatorname{dim} \mathbb{H}_{1}(\Gamma, \mathbb{Z})=b_{1}(\Gamma)+|E(\Gamma)|=2|E(\Gamma)|-|V(\Gamma)|+1,
$$

and its number of extremal rays is equal to $2|E(\Gamma)|$ by Lemma $5.1\left(\right.$ iii). Therefore, $\sigma_{\Gamma}^{\vee}$ is simplicial if and only if $\Gamma$ has a unique vertex, and we are done.

Denote by $\underline{0}$ the unique torus fixed point of the affine toric variety $X_{\Gamma}$, and let $\mathfrak{m}$ the maximal ideal of $U(\Gamma)$ corresponding to it. Explicitly, under the isomorphism $U(\Gamma) \cong D(\Gamma)$ of Theorem 4.6, the ideal $\mathfrak{m}$ is generated by the variables $X^{z}$ and $T_{e}$. The dimension of the tangent space of $X_{\Gamma}$ at $\underline{0}$ or, equivalently, the embedded dimension of $U(\Gamma)$ at $\mathfrak{m}$, is easy to determine in terms of the (unoriented) circuits $\operatorname{Cir}(\Gamma)$ of $\Gamma$ and the loops $\operatorname{Loops}(\Gamma)$ of $\Gamma$.

Proposition 5.8. The Zariski tangent space $T_{\underline{0}}\left(X_{\Gamma}\right)$ at $\underline{0}$ has dimension equal to

$$
\operatorname{dim} T_{\underline{0}}\left(X_{\Gamma}\right)=2|\operatorname{Cir}(\Gamma)|+|E(\Gamma)|-|\operatorname{Loops}(\Gamma)| .
$$

Proof. By [CKV13, Theorem 4.15(i), Proposition 5.2], the embedded dimension of $R\left(\Gamma^{d}, \phi^{d}\right)$ at $\mathfrak{m}$ is equal to the cardinality of the set $\operatorname{Cir}_{\phi^{d}}\left(\Gamma^{d}\right)$ of oriented circuits compatibly oriented with $\phi^{d}$. Therefore, we conclude by applying Proposition 3.3 and the lemma below.

Lemma 5.9. The set $\operatorname{Cir}_{\phi^{d}}\left(\Gamma^{d}\right)$ of oriented circuits compatibly oriented with $\phi^{d}$ is equal to

$$
\left|\operatorname{Cir}_{\phi^{d}}\left(\Gamma^{d}\right)\right|=2|\operatorname{Cir}(\Gamma)|+|E(\Gamma)|-|\operatorname{Loops}(\Gamma)| \text {. }
$$


Proof. For every $e \in E(\Gamma) \backslash \operatorname{Loops}(\Gamma)$, we set $\eta_{e}:=\phi^{d}\left(e^{\prime}\right)+\phi^{d}\left(e^{\prime \prime}\right) \in \operatorname{Cir}_{\phi^{d}}\left(\Gamma^{d}\right)$, where $e^{\prime}$ and $e^{\prime \prime}$ are the two edges of $\Gamma^{d}$ corresponding to $e \in \Gamma$. By taking the image of a circuit of $\Gamma^{d}$ under the natural contraction map $\Gamma^{d} \rightarrow \Gamma$, we get a well-defined map

$$
\operatorname{Cir}_{\phi^{d}}\left(\Gamma^{d}\right) \backslash\left\{\eta_{e}: e \in E(\Gamma) \backslash \operatorname{Loops}(\Gamma)\right\} \rightarrow \operatorname{Cir}(\Gamma) .
$$

Since any circuit of $\Gamma$ can be lifted in exactly two ways to an oriented circuit of $\Gamma^{d}$ compatibly oriented with $\phi^{d}$, the above map is surjective and $2: 1$. This concludes the proof.

Remark 5.10. In terms of Theorem 4.6, Proposition 5.8 reflects the fact that $D(\Gamma)$ is generated by $2|\operatorname{Cir}(\Gamma)|$ variables $X$ and $|E(\Gamma)|$ variables $T$ and has $|\operatorname{Loops}(\Gamma)|$ relations involving linear terms.

We now consider the multiplicity of $X_{\Gamma}$ at $\underline{0}$. To that aim, we need to recall some definitions. Let $H_{\mathbb{Z}}$ be a lattice, and let $\sigma$ be a strongly convex rational polyhedral cone in $H_{\mathbb{R}}=H_{\mathbb{Z}} \otimes \mathbb{R}$. Set $C(\sigma):=\sigma \cap H_{\mathbb{Z}}$, let $H_{\mathbb{R}, \sigma}=\langle\sigma\rangle \subseteq H_{\mathbb{R}}$ be the linear span of $\sigma$ in $H_{\mathbb{R}}$, and set $H_{\mathbb{Z}, \sigma}:=\langle\sigma\rangle \cap H_{\mathbb{Z}}$. Note that $H_{\mathbb{R}, \sigma}=H_{\mathbb{Z}, \sigma} \otimes \mathbb{R}$. We denote by $\operatorname{vol}_{C(\sigma)}$ the unique translation-invariant measure on $H_{\mathbb{R}, \sigma}$ such that the volume of a standard unimodular simplex $\Delta$ is 1 (that is, $\Delta$ is the convex hull of a basis of $H_{\mathbb{Z}, \sigma}$ together with 0). Following [GKZ94, Chapter 5, Section E, p. 184], denote by $K_{+}(C(\sigma)) \subseteq H_{\mathbb{R}, \sigma}$ the convex hull of the set $C(\sigma) \backslash\{0\}$ and by $K_{-}(C(\sigma))$ the closure of $\sigma \backslash K_{+}(C(\sigma))$. The set $K_{-}(C(\sigma))$ is a bounded (possibly not convex) lattice polyhedron in $H_{\mathbb{R}, \sigma}$ which is called the subdiagram part of $C(\sigma)$.

Definition 5.11 ([GKZ94, Chapter 5, Definition 3.8]). The subdiagram volume of $C(\sigma)$ is the natural number

$$
u(C(\sigma)):=\operatorname{vol}_{C(\sigma)}\left(K_{-}(C(\sigma))\right)
$$

Now, let $R(\sigma)=R(C(\sigma))$ be the semigroup ring associated with $C(\sigma)$. Let $\mathfrak{m}$ be the maximal ideal generated by the generators of the $k$-algebra $R(\sigma)$. Let $\underline{0}$ be the corresponding point in $X_{\sigma}:=\operatorname{Spec} R(\sigma)$. The multiplicity of $X_{\sigma}$ at $\underline{0}$ is given by (see, for example, [GKZ94, Chapter 5, Theorem 3.14])

$$
\operatorname{mult}_{\underline{0}} X_{\sigma}=u(C(\sigma)) .
$$

Theorem 5.12. Let $\Gamma$ be a graph, and let $\sigma=\sigma_{\Gamma}$. Then mult ${ }_{0} X_{\Gamma}=u(C(\sigma))$.

Remark 5.13. It would be interesting to have a formula for mult ${ }_{0} X_{\Gamma}$ in terms of standard invariants of the graph $\Gamma$ (or $\left.\Gamma^{d}\right)$.

\section{The cographic toric ring $U(\Gamma)$ as a ring of invariants}

In this section, we show that the cographic toric ring $U(\Gamma)$ appears as ring of invariants of a torus acting on a certain polynomial ring. Indeed, this invariant ring will appear in Section 8 in the description of the completed local rings of the compactified universal Jacobian.

Consider the action of the algebraic torus $T_{\Gamma}:=\prod_{v \in V(\Gamma)} \mathbb{G}_{m}$ on the polynomial ring

$$
B(\Gamma):=k\left[X_{\vec{e}}: \vec{e} \in \vec{E}\right]
$$

given by the rule that $\lambda=\left(\lambda_{v}\right)_{v \in V(\Gamma)} \in T_{\Gamma}$ acts as

$$
\lambda \cdot X_{\vec{e}}=\lambda_{s(\vec{e})} \lambda_{t(\vec{e})}^{-1} X_{\vec{e}} .
$$




\section{S. Casalaina-Martin, J. L. Kass and F. Viviani}

In order to more easily connect the results of this paper to those in [CKV15], we note the following.

Remark 6.1. The ring $B(\Gamma)$ is isomorphic to

$$
\frac{k\left[X_{e}, Y_{e}, T_{e}: e \in E(\Gamma)\right]}{\left(X_{e} Y_{e}-T_{e}\right)}
$$

and its completion at the maximal ideal $\left(X_{e}, Y_{e}\right)$ is isomorphic to the ring denoted by $\widehat{B(\Gamma)}$ in [CKV15, Theorem A]. Under this isomorphism, the action of $T_{\Gamma}$ on $B(\Gamma)$ given above induces the same action of $T_{\Gamma}$ on $\widehat{B(\Gamma)}$ as that given in [CKV15, Theorem A].

TheOREM 6.2. The cographic toric ring $U(\Gamma)$ is isomorphic to the subring $B(\Gamma)^{T_{\Gamma}} \subset B(\Gamma)$ of $T_{\Gamma}$-invariants on $B(\Gamma)$.

Proof. Using Theorem 4.6, we are going to show that $B(\Gamma)^{T_{\Gamma}}$ is isomorphic to the $k$-algebra $D(\Gamma)$. The proof is essentially identical to the proof of [CKV13, Theorem 6.1]; we first show that the underlying $k$-vector spaces agree, and then we show that the multiplication rules agree. In keeping with the notation of the proof of [CKV13, Theorem 6.1], we first observe (as in Remark 6.1) that $B(\Gamma)$ can be identified with

$$
\frac{k\left[X_{\overleftarrow{e}}, X_{\vec{e}}, T_{e}: e \in E(\Gamma)\right]}{\left(X_{\overleftarrow{e}} X_{\vec{e}}-T_{e}\right)} .
$$

The key point is then to identify the invariant monomials in this ring. This is made easier by the observation that every monomial has an expression of the form

$$
\prod_{e \in E(\Gamma)} X_{\vec{e}}^{a_{e}} T_{e}^{b_{e}}
$$

with $a_{e}, b_{e} \in \mathbb{Z}_{\geqslant 0}$, where for each $e \in E(\Gamma)$ we have that $\vec{e}$ is one of the two orientations of $e$. The expression is unique up to replacing $\vec{e}$ with $\overleftarrow{e}$ for those $e$ such that $a_{e}=0$. The same direct analysis of the action as in the proof of [CKV13, Theorem 6.1] shows that in order for this monomial to be invariant, we must have $\sum_{e \in E(\Gamma)} a_{e} \vec{e} \in H_{1}(\Gamma, \mathbb{Z})$. Thus, as $k$-vector spaces, $D(\Gamma)$ and $B(\Gamma)^{T_{\Gamma}}$ agree. It remains to check that multiplication agrees. This can be checked at the level of monomials, and $\psi$ in the definition of $D(\Gamma)$ (see Remark 4.1) was constructed exactly to make these agree.

The cographic toric ring $U(\Gamma)$ is related to the cographic toric face ring $R(\Gamma)$ studied in [CKV13], as explained in the following remark (see also Remark 4.7).

Remark 6.3. The action of $T_{\Gamma}$ on $B(\Gamma)$ defines an action on the quotient

$$
A(\Gamma):=\frac{B(\Gamma)}{\left(X_{\vec{e}} X_{\overleftarrow{e}}: e \in E(\Gamma)\right)} \cong \frac{k\left[X_{e}, Y_{e}: e \in E(\Gamma)\right]}{\left(X_{e} Y_{e}\right)},
$$

which coincides with the action of $T_{\Gamma}$ defined in [CKV13, Theorem A]. Therefore, the natural surjection $B(\Gamma) \rightarrow A(\Gamma)$ induces, by taking $T_{\Gamma}$-invariants, a map

$$
U(\Gamma) \rightarrow R(\Gamma)
$$

where $R(\Gamma):=A(\Gamma)^{T_{\Gamma}}$ is the cographic toric face ring of $\Gamma$ (see [CKV13, Theorem 6.1]). Indeed, the morphism (6.2) coincides with the morphism (4.4), and, in particular, it is surjective. 


\section{Examples}

We now include a few examples of cographic toric rings.

\subsection{The $n$-cycle $C_{n}$}

Let $C_{n}$ be the $n$-cycle graph, that is, the graph formed by $n$ vertices connected by a closed chain of $n$ edges, as depicted in Figure 3.

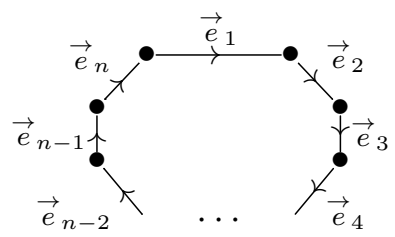

Figure 3 . The $n$-cycle $C_{n}$ with half of its oriented edges

The cographic toric ring of $C_{n}$ admits the following explicit presentation:

$$
U\left(C_{n}\right)=\frac{k\left[X, Y, T_{1}, \ldots, T_{n}\right]}{\left(X Y-T_{1} \cdots T_{n}\right)} .
$$

To see this, consider the explicit presentation of the cographic toric ring given in Section 4 . Note that there are two oriented circuits of $C_{n}$ giving rise to the elements $c:=\left[\vec{e}_{1}\right]+\cdots+\left[\vec{e}_{n}\right]$ and $-c$ of $H_{1}(\Gamma, \mathbb{Z})$. Then, by Proposition 5.8, the generators of the ring $U\left(C_{n}\right)$ are $X=X^{c}$, $Y=X^{-c}$, and $T_{i}=T_{e_{i}}$ for $1 \leqslant i \leqslant n$. Since the function $\psi$ of $(4.1)$ on $H_{1}\left(C_{n}, \mathbb{Z}\right)$ is such that $\psi(c,-c)=e_{1}+\cdots+e_{n}$, we get the relation $X Y-T_{1} \cdots T_{n}=0$.

It is easily checked that the cographic toric variety $X_{C_{n}}=\operatorname{Spec} U\left(C_{n}\right)$ satisfies

$$
\begin{aligned}
\operatorname{dim} X_{C_{n}} & =n+1, \\
\operatorname{dim} T_{\underline{0}}\left(X_{C_{n}}\right) & =n+2, \\
\operatorname{mult}_{\underline{0}} X_{C_{n}} & =2,
\end{aligned}
$$

which is, of course, in agreement with the formulas obtained in Section 5.

\subsection{The $n$-thick edge $I_{n}$}

Let $I_{n}$ be the $n$th thick edge graph, that is, the graph formed by two vertices joined by $n$ edges, as depicted in Figure 4.

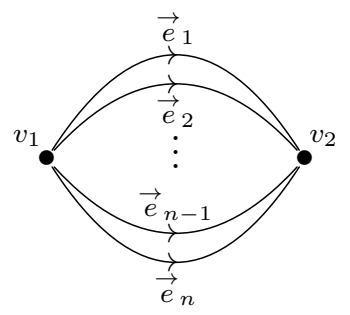

Figure 4 . The $n$-thick edge $I_{n}$ with half of its oriented edges 


\section{S. Casalaina-Martin, J. L. Kass and F. Viviani}

The cographic toric ring of $I_{n}$ admits the following explicit presentation:

$$
U\left(I_{n}\right)=\frac{k\left[X_{i j}, T_{k}\right]_{1 \leqslant i \neq j \leqslant n, 1 \leqslant k \leqslant n}}{\left(X_{i j} X_{j i}-T_{i} T_{j}, X_{i j} X_{j k}-T_{j} X_{i k}\right)} .
$$

To see this, consider the explicit presentation of the cographic toric ring given in Section 4 . Note that the oriented circuits of $I_{n}$ gives rise to the elements $\gamma_{i j}:=\left[\vec{e}_{i}\right]-\left[\vec{e}_{j}\right] \in H_{1}\left(I_{n}, \mathbb{Z}\right)$ for any $1 \leqslant i<j \leqslant n$. Then, using Proposition 5.8, we deduce that the generators of the ring $U\left(I_{n}\right)$ are $X_{i j}=X^{\gamma_{i j}}$ for $1 \leqslant i \neq j \leqslant n$ and $T_{k}=T_{e_{k}}$ for $1 \leqslant k \leqslant n$. Since the only non-zero values of the function $\psi$ of (4.1) on the oriented circuits $\gamma_{i j}$ of $H_{1}\left(I_{n}, \mathbb{Z}\right)$ are given by

$$
\begin{aligned}
\psi\left(\gamma_{i j}, \gamma_{j i}\right) & =e_{i}+e_{j}, \\
\psi\left(\gamma_{i j}, \gamma_{j k}\right) & =e_{j},
\end{aligned}
$$

we get the desired relations among the given generators.

It is easily checked that the cographic toric variety $X_{I_{n}}=\operatorname{Spec} U\left(I_{n}\right)$ satisfies

$$
\begin{aligned}
\operatorname{dim} X_{I_{n}} & =2 n-1, \\
\operatorname{dim} T_{\underline{0}}\left(X_{I_{n}}\right) & =n^{2}, \\
\operatorname{mult}_{\underline{0}} X_{I_{n}} & =\left(\begin{array}{c}
2(n-1) \\
n-1
\end{array}\right),
\end{aligned}
$$

which is, of course, in agreement with the formulas obtained in Section 5.

\section{The compactified universal Jacobian}

The aim of this section is to apply the results of the previous sections in order to study the singularities of the compactified universal Jacobian $\bar{J}_{d, g}$ and eventually prove in Theorem 8.4 that $\bar{J}_{d, g}$ has canonical singularities over a base field $k$ of characteristic zero, at least if $g \geqslant 4$. We then deduce some consequences for the birational geometry of the universal Jacobians $J_{d, g}$. The outline of this section is as follows. In Section 8.1, we relate the local rings of the compactified universal Jacobian to the rings appearing earlier in this paper. The culmination is Theorem 8.1, which essentially reduces the problem to studying finite quotients of the cographic rings $U(\Gamma)$. In order to describe this quotient, it is convenient to compare with an associated quotient obtained from the local structure of $\bar{M}_{g}$; this comparison is made in Section 8.2, culminating in Theorem 8.2. In Section 8.4, we give the proof of Theorem 8.4. The argument relies on a generalization of the Reid-Tai-Shepherd-Barron criterion to singular toric varieties, which can be found in the appendix. Consequences for the birational geometry of $\bar{J}_{d, g}$ are given in Section 8.5.

\subsection{The local rings of $\bar{J}_{d, g}$}

In this subsection, which is heavily based on our previous work [CKV15], we obtain an explicit description of the completed local rings of $\bar{J}_{d, g}$ in terms of the cographic toric rings studied in the previous sections.

Fix a point $(C, I) \in \bar{J}_{d, g}$; that is, $C$ is a stable curve of genus $g$, and $I$ is a rank one, torsionfree sheaf of degree $d$ on $C$, which is polystable with respect to the canonical polarization $\omega_{C}$. Let $\Sigma_{(C, I)}$ (or simply $\Sigma$ when the pair $(C, I)$ we are dealing with is clear from the context) be the set of nodes of $C$ where $I$ is not locally free. Let $\Gamma_{(C, I)}$ (or simply $\Gamma$ when the pair $(C, I)$ we are dealing with is clear from the context) be the graph obtained from the dual graph of $C$ by contracting the edges corresponding to the nodes that are not in $\Sigma_{(C, I)}$. In particular, the edges 


\section{THE SINGULARITIES AND BIRATIONAL GEOMETRY}

of $\Gamma_{(C, I)}$ correspond naturally to the nodes in $\Sigma_{(C, I)}$. Note that $\Gamma_{(C, I)}$ is the dual graph of the curve obtained from $C$ by smoothing the nodes at which $I$ is locally free. For convenience, we fix an arbitrary orientation of $\Gamma_{(C, I)}$, and we denote by $s, t: E\left(\Gamma_{(C, I)}\right) \rightarrow V\left(\Gamma_{(C, I)}\right)$ the source and target maps associating with any edge of $\Gamma_{(C, I)}$ the source and target with respect to the chosen orientation.

We now review the deformation theory of the pair $(C, I)$, referring to [CKV15] for more details and proofs. As explained in $[\mathrm{CKV} 15, \S 3]$, the deformation functor $\operatorname{Def}_{(C, I)}$ of the pair $(C, I)$ fits into the sequence

$$
\operatorname{Def}_{(C, I)}^{\text {l.t. }} \longrightarrow \operatorname{Def}_{(C, I)} \stackrel{F}{\longrightarrow} \prod_{e \in \Sigma} \operatorname{Def}_{\left(C_{e}, I_{e}\right)}=\operatorname{Def}_{(C, I)}^{\text {loc }},
$$

where $\operatorname{Def}_{\left(C_{e}, I_{e}\right)}$ is the deformation functor of the pair consisting of $C_{e}:=\operatorname{Spec} \widehat{\mathscr{O}}_{C, e}$ and the pull-back $I_{e}$ of $I$ to $C_{e}$, the map $F$ is the forgetful map taking deformations of $(C, I)$ to local deformations at the set of nodes $e \in \Sigma$ where $I$ fails to be locally free, and $\operatorname{Def}_{(C, I)}^{\text {l.t. is the }}$ subfunctor of $\operatorname{Def}_{(C, I)}$ parametrizing locally trivial deformations, that is, deformations of $(C, I)$ that map to the trivial deformation via the forgetful map $F$. The above three deformation functors are unobstructed and the forgetful map $F$ is formally smooth (see [CKV15, §3]). In particular, we get an exact sequence of tangent spaces

$$
0 \rightarrow T \operatorname{Def}_{(C, I)}^{\text {l.t. }} \rightarrow T \operatorname{Def}_{(C, I)} \rightarrow T \operatorname{Def}_{(C, I)}^{\text {loc }} \rightarrow 0
$$

Define the $k$-algebra

$$
R_{(C, I)}:=k\left[T^{\vee} \operatorname{Def}_{(C, I)}\right]=\bigoplus_{n \in \mathbb{N}} \operatorname{Sym}^{n} T^{\vee} \operatorname{Def}_{(C, I)},
$$

where $T^{\vee} \operatorname{Def}_{(C, I)}$ is the dual of the tangent space $T \operatorname{Def}_{(C, I)}$. Fixing a splitting of the exact sequence (8.2) and using the explicit description of a mini-versal deformation ring for $\operatorname{Def}_{\left(X_{e}, I_{e}\right)}$ obtained in [CKV15, Lemma 3.14], we can write $R_{(C, I)}$ in the following form:

$$
\begin{aligned}
R_{(C, I)} & =k\left[T^{\vee} \operatorname{Def}_{(C, I)}^{\text {loc }}\right] \otimes_{k} k\left[T^{\vee} \operatorname{Def}_{(C, I)}^{\text {l.t. }}\right] \\
& =\bigotimes_{e \in \Sigma} \frac{k\left[X_{e}, Y_{e}, T_{e}\right]}{\left(X_{e} Y_{e}-T_{e}\right)} \otimes_{k} k\left[T^{\vee} \operatorname{Def}_{(C, I)}^{\text {l.t. }}\right]=B(\Gamma) \otimes_{k} k\left[T^{\vee} \operatorname{Def}_{(C, I)}^{\text {l.t. }}\right],
\end{aligned}
$$

where $B(\Gamma)$ is the ring defined in Remark 6.1. As proved in [CKV15, §3.2], the mini-versal deformation ring of the functor $\operatorname{Def}_{(C, I)}$ is given by the completion $\widehat{R}_{(C, I)}$ of $R_{(C, I)}$ at the maximal ideal $\mathfrak{m}_{0}$ generated by $T^{\vee} \operatorname{Def}_{(C, I)}$. Geometrically, the variables $X_{e}$ and $Y_{e}$ correspond to the deformations of $I$ at the node $e \in E(\Gamma)=\Sigma$, and the variable $T_{e}$ corresponds to the smoothing of $C$ at $e$. Note also that the completion $\widehat{B}(\Gamma)$ of $B(\Gamma)$ at the maximal ideal generated by $T^{\vee} \operatorname{Def}_{(C, I)}^{\text {loc }}$ was shown to be mini-versal for $\operatorname{Def}_{(C, I)}^{\text {loc }}$; for this reason, we will sometimes also write

$$
R_{(C, I)}^{\mathrm{loc}}:=B(\Gamma)=\bigotimes_{e \in \Sigma} \frac{k\left[X_{e}, Y_{e}, T_{e}\right]}{\left(X_{e} Y_{e}-T_{e}\right)} .
$$

Now, consider the automorphism group $\operatorname{Aut}(C, I)$ of $(C, I)$, consisting of all pairs $(\sigma, \tau)$ such that $\sigma: C \stackrel{\cong}{\longrightarrow} C$ is an automorphism of $C$ and $\tau: I \stackrel{\cong}{\longrightarrow} \sigma^{*}(I)$ is an isomorphism of sheaves on $C$. We have a natural exact sequence of groups

$$
\{1\} \longrightarrow \operatorname{Aut}(I) \stackrel{i}{\longrightarrow} \operatorname{Aut}(C, I) \stackrel{p}{\longrightarrow} \operatorname{Stab}_{C}(I) \longrightarrow\{1\},
$$

where $\operatorname{Stab}_{C}(I) \subseteq \operatorname{Aut}(C)$ is the subgroup of $\operatorname{Aut}(C)$ (which is finite since $C$ is stable) consisting 


\section{S. Casalaina-Martin, J. L. Kass and F. Viviani}

of all the elements $\sigma \in \operatorname{Aut}(C)$ such that $\sigma^{*}(I) \cong I$. The group $\operatorname{Aut}(I)$ is an algebraic torus, which by [CKV15, Remark 5.9] is naturally isomorphic to

$$
\operatorname{Aut}(I)=T_{\Gamma}:=\prod_{v \in V(\Gamma)} \mathbb{G}_{m} .
$$

The automorphism group $\operatorname{Aut}(C, I)$ acts naturally on $\operatorname{Def}_{(C, I)}$ (see [CKV15, Definition 3.4]); hence it acts also on the tangent space $T \operatorname{Def}_{(C, I)}$, and this action clearly preserves the exact sequence (8.2). Therefore, we get a natural linear action of $\operatorname{Aut}(C, I)$ on $R_{(C, I)}$ which preserves the decomposition of $R_{(C, I)}$ given in (8.4). It follows from [CKV15, $\S 5$ ] that the induced action of the subgroup $\operatorname{Aut}(I)$ is trivial on $k\left[T^{\vee} \operatorname{Def}_{(C, I)}^{\text {l.t. }}\right]$ and coincides with the action of $T_{\Gamma}$ on $B(\Gamma)$ given by (6.1) after the identification of Remark 6.1. Explicitly, an element $\lambda=\left(\lambda_{v}\right)_{v \in V(\Gamma)} \in T_{\Gamma}$ acts on the generators of $B(\Gamma)$ as

$$
\lambda \cdot X_{e}=\lambda_{s(e)} \lambda_{t(e)}^{-1} X_{e}, \quad \lambda \cdot Y_{e}=\lambda_{s(e)}^{-1} \lambda_{t(e)} Y_{e}, \quad \text { and } \quad \lambda \cdot T_{e}=T_{e} .
$$

The subring $R_{(C, I)}^{\operatorname{Aut}(C, I)} \subseteq R_{(C, I)}$ of invariants for the action of $\operatorname{Aut}(C, I)$ on $R_{(C, I)}$ can be computed in two steps. We first take the subring $R_{(C, I)}^{\operatorname{Aut}(I)} \subset R_{(C, I)}$ of invariants for the subgroup $\operatorname{Aut}(I)$. Then, we take the invariants for the induced action of the finite group $\operatorname{Stab}_{C}(I)$ on $R_{(C, I)}^{\operatorname{Aut}(I)}$. By Theorem 6.2, the ring of invariants with respect to $\operatorname{Aut}(I)$ is equal to

$$
R_{(C, I)}^{\mathrm{Aut}(I)}=U(\Gamma) \otimes_{k} k\left[T^{\vee} \operatorname{Def}_{(C, I)}^{1 . t .}\right]
$$

where $U(\Gamma)$ is the cographic toric ring associated with $\Gamma$. Therefore, the subring of invariants with respect to $\operatorname{Aut}(C, I)$ is given by

$$
R_{(C, I)}^{\operatorname{Aut}(C, I)}=\left(R_{(C, I)}^{\operatorname{Aut}(I)}\right)^{\operatorname{Stab}_{C}(I)}=\left(U(\Gamma) \otimes_{k} k\left[T^{\vee} \operatorname{Def}_{(C, I)}^{\text {l.t. }}\right]\right)^{\operatorname{Stab}_{C}(I)} .
$$

Next, we show that the completion of the invariant subring (8.9) at the maximal ideal $\mathfrak{m}_{0} \cap$ $R_{(C, I)}^{\text {Aut }(C, I)}$ gives a description of the completed local ring $\widehat{\mathcal{O}}_{\bar{J}_{d, g},(C, I)}$ of the universal compactified Jacobian $\bar{J}_{d, g}$ at $(C, I)$.

TheOREm 8.1. We use the same notation as above. Assume that $\operatorname{Stab}_{C}(I)$ does not contain elements of order equal to $p=\operatorname{char}(k)$. The completion of the invariant subring $R_{(C, I)}^{\operatorname{Aut}(C, I)}$ at the maximal ideal $\mathfrak{m}_{0} \cap R_{(C, I)}^{\mathrm{Aut}(C, I)}$ is isomorphic to the completed local ring $\widehat{\mathcal{O}}_{\bar{J}_{d, g},(C, I)}$ of the compactified universal Jacobian $\bar{J}_{d, g}$ at $(C, I)$.

Proof. The linear action of $\operatorname{Aut}(C, I)$ on $R_{(C, I)}$ described above induces a unique action on the completion $\widehat{R}_{(C, I)}$ of $R_{(C, I)}$ at the maximal ideal $\mathfrak{m}_{0}$. Since $\operatorname{Aut}(C, I)$ is a linearly reductive group (by our assumption on $\operatorname{Stab}_{C}(I)$ ), the formation of $\operatorname{Aut}(C, I)$-invariants commutes with completion (see, for example, [CKV15, Lemma 6.7]); in symbols,

$$
\left(R_{C, I}^{\operatorname{Aut}(C, I)}\right)^{\widehat{N}} \cong\left(\widehat{R}_{(C, I)}\right)^{\operatorname{Aut}(C, I)}
$$

where on the right-hand side, the completion is taken with respect to the maximal ideal $\mathfrak{m}_{0}$ of $\widehat{R}_{(C, I)}$ generated by $T^{\vee} \operatorname{Def}_{(C, I)}$ and on the left the completion is taken with respect to the maximal ideal $\mathfrak{m}_{0} \cap R_{(C, I)}^{\mathrm{Aut}(C, I)}$.

As observed before, the ring $\widehat{R}_{(C, I)}$ is the mini-versal deformation ring of the functor $\operatorname{Def}_{(C, I)}$, 
which means that there is a formally smooth natural transformation of functors

$$
\Phi: \quad \operatorname{Spf} \widehat{R}_{(C, I)} \rightarrow \operatorname{Def}_{(C, I)},
$$

whose associated map on tangent spaces

$$
T \Phi: T \operatorname{Spf} \widehat{R}_{(C, I)} \rightarrow T \operatorname{Def}_{(C, I)}
$$

is an isomorphism. Explicitly, the isomorphism $T \Phi$ is obtained by first identifying the tangent space of Spf $\widehat{R}_{(C, I)}$ with the tangent space $T_{\mathfrak{m}_{0}} R_{(C, I)}=\left(\mathfrak{m}_{0} / \mathfrak{m}_{0}^{2}\right)^{\vee}$ of the ring $R_{(C, I)}$ at $\mathfrak{m}_{0}$ and then identifying $T_{\mathfrak{m}_{0}} R_{(C, I)}$ with $T \operatorname{Def}_{(C, I)}$ using the definition (8.3) of $R_{(C, I)}$.

Now, observe that our specified linear action of $\operatorname{Aut}(C, I)$ on $R_{(C, I)}$ is defined in such a way that the isomorphism $T \Phi$ becomes $\operatorname{Aut}(C, I)$-equivariant. By Rim's arguments [Rim80], the $\operatorname{Aut}(C, I)$-equivariance of $T \Phi$ implies that $\Phi$ is also $\operatorname{Aut}(C, I)$-equivariant; hence the specified action of $\operatorname{Aut}(C, I)$ on $\widehat{R}_{(C, I)}$ is the unique action that makes $\Phi$ equivariant, according to Rim's theorem (see [CKV15, Fact 5.4]). Therefore, we can apply [CKV15, Theorem 6.1(ii)] in order to conclude that

$$
\widehat{\mathcal{O}}_{\bar{J}_{d, g},(C, I)} \cong \widehat{R}_{(C, I)}^{\mathrm{Aut}(C, I)} \text {. }
$$

The proof of the theorem follows by combining (8.10) and (8.13).

\subsection{The local structure of the morphism $\pi: \bar{J}_{d, g} \rightarrow \bar{M}_{g}$}

The aim of this subsection is to study the local structure of the morphism $\pi: \bar{J}_{d, g} \rightarrow \bar{M}_{g}$ around a point $(C, I) \in \bar{J}_{d, g}$, where we assume as usual that $I$ is polystable with respect to $\omega_{C}$.

First of all, there is a natural forgetful morphism $\Pi: \operatorname{Def}_{(C, I)} \rightarrow \operatorname{Def}_{C}$, from the deformation functor of the pair $(C, I)$ to the deformation functor of $C$, which is equivariant with respect to the group homomorphism $\operatorname{Aut}(C, I) \rightarrow \operatorname{Aut}(C)$ and the natural actions of $\operatorname{Aut}(C, I)$ on $\operatorname{Def}_{(C, I)}$ and of $\operatorname{Aut}(C)$ on $\operatorname{Def}_{C}$ (see [CKV15, Definition 3.4]). The forgetful morphism $\Pi$ fits into the diagram

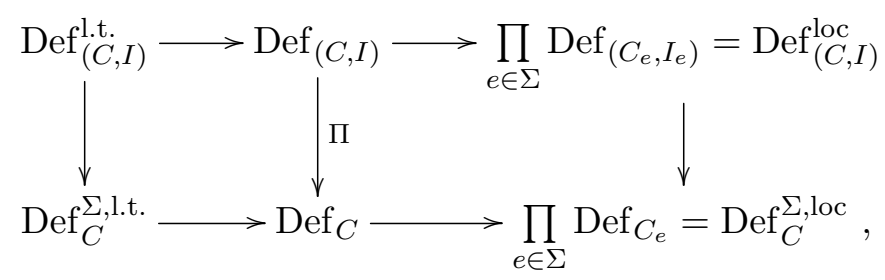

where $\operatorname{Def}_{C}^{\Sigma \text {,loc }}$ is the local deformation functor of $C$ at the nodes $\Sigma=\Sigma_{(C, I)}$ of $C$ where $I$ is not invertible and $\operatorname{Def}_{C}^{\Sigma \text {,l.t. }}$ is the subfunctor of $\operatorname{Def}_{C}$ parametrizing deformations of $C$ that are locally trivial around the nodes of $\Sigma$. Passing to the tangent spaces, we get the following diagram with exact rows:

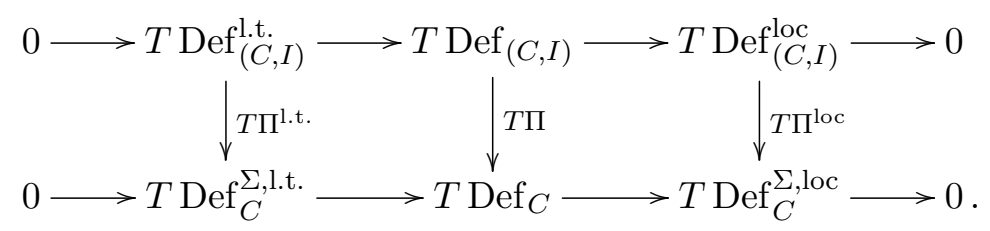

Observe that the map $T \Pi^{\text {l.t. }}$ is surjective and its kernel can be naturally identified with the tangent space $T \operatorname{Def}_{L}$ of the deformation functor $\operatorname{Def}_{L}$, where $L$ is the line bundle on the partial normalization $g: C_{\Sigma} \rightarrow C$ at the nodes of $\Sigma=\Sigma_{(C, L)}$ such that $g_{*}(L)=I$ (see [CKV15, Lemma 3.16]). 


\section{S. Casalaina-Martin, J. L. Kass and F. Viviani}

Fixing a splitting of the second row of (8.15), we define the $k$-algebra

$$
R_{C}:=k\left[T^{\vee} \operatorname{Def}_{C}\right]=k\left[T^{\vee} \operatorname{Def}_{C}^{\Sigma, \text { loc }}\right] \otimes_{k} k\left[T^{\vee} \operatorname{Def}_{C}^{\Sigma, \text { l.t. }}\right]=\bigotimes_{e \in \Sigma} k\left[T_{e}\right] \otimes_{k} k\left[T^{\vee} \operatorname{Def}_{C}^{\Sigma, \text { l.t. }}\right],
$$

where the variable $T_{e}$ corresponds to the smoothing of $C$ at $e$. Observe that the finite group Aut $(C)$ acts linearly on $R_{C}$, via its natural action on $T \operatorname{Def}_{C}$. The diagram (8.15), after choosing compatible splittings of the horizontal rows and of the left vertical column, gives rise to an injective morphism of $k$-algebras

$$
\begin{aligned}
R_{C}=\bigotimes_{e \in \Sigma} k\left[T_{e}\right] \otimes_{k} k\left[T^{\vee} \operatorname{Def}_{C}^{\Sigma, \text { l.t.t. }] \hookrightarrow R_{(C, I)}}\right. & =\bigotimes_{e \in \Sigma} \frac{k\left[X_{e}, Y_{e}, T_{e}\right]}{\left(X_{e} Y_{e}-T_{e}\right)} \otimes_{k} k\left[T^{\vee} \operatorname{Def}_{(C, I)}^{\text {l.t. }}\right]= \\
& =\bigotimes_{e \in \Sigma} \frac{k\left[X_{e}, Y_{e}, T_{e}\right]}{\left(X_{e} Y_{e}-T_{e}\right)} \otimes_{k} k\left[T^{\vee} \operatorname{Def}_{C}^{\Sigma, \text { l.t.t. }}\right] \otimes_{k} k\left[T^{\vee} \operatorname{Def}_{L}\right] .
\end{aligned}
$$

Now, consider the action of Aut $(I)$ on $R_{(C, I)}$ as in Section 8.1. From (8.7), it follows that each $T_{e}$ is invariant under the action of $\operatorname{Aut}(I)$, so that the inclusion (8.17) factors through

$$
R_{C}=\bigotimes_{e \in \Sigma} k\left[T_{e}\right] \otimes_{k} k\left[T^{\vee} \operatorname{Def}_{C}^{\Sigma, \text { l.t. }}\right] \hookrightarrow R_{(C, I)}^{\mathrm{Aut}(I)}=U(\Gamma) \otimes_{k} k\left[T^{\vee} \operatorname{Def}_{C}^{\Sigma, \text { l.t.t. }}\right] \otimes_{k} k\left[T^{\vee} \operatorname{Def}_{L}\right] .
$$

Note that the finite subgroup $\operatorname{Stab}_{C}(I)$ acts in a compatible way on both rings in (8.18), while the bigger finite group $\operatorname{Aut}(C)$ acts only on the ring on the left.

Theorem 8.2. We use the same notation as above. Assume that $\operatorname{Aut}(C)$ does not contain elements of order equal to $p=\operatorname{char}(k)$. The inclusion of complete local rings

$$
\widehat{\mathcal{O}}_{\bar{M}_{g}, C} \hookrightarrow \widehat{\mathcal{O}}_{\bar{J}_{d, g},(C, I)}
$$

induced by the surjective morphism $\pi: \bar{J}_{d, g} \rightarrow \bar{M}_{g}$ coincides with the completion of the inclusion

$$
R_{C}^{\mathrm{Aut}(C)} \hookrightarrow R_{(C, I)}^{\mathrm{Aut}(C, I)}=\left(R_{(C, I)}^{\mathrm{Aut}(I)}\right)^{\operatorname{Stab}_{C}(I)}
$$

induced by (8.18) at the maximal ideals $\mathfrak{m}_{0} \cap R_{C}^{\mathrm{Aut}(C)}$ and $\mathfrak{m}_{0} \cap R_{(C, I)}^{\mathrm{Aut}(C, I)}$, respectively.

Proof. The assumption on the order of the elements of $\operatorname{Aut}(C)$ implies that $\operatorname{Aut}(C)$ and $\operatorname{Aut}(C, I)$ are linearly reductive groups. Since the formation of invariants under the action of a linear reductive group commutes with completion (see, for example, [CKV15, Lemma 6.7]), the completion of the inclusion (8.19) is equal to the inclusion

$$
\widehat{R}_{C}^{\mathrm{Aut}(C)} \hookrightarrow \widehat{R}_{(C, I)}^{\mathrm{Aut}(C, I)},
$$

where the completions, done with respect to the maximal ideals $\mathfrak{m}_{0} \cap R_{C}$ and $\mathfrak{m}_{0}$, respectively, are acted upon naturally by $\operatorname{Aut}(C)$ and $\operatorname{Aut}(C, I)$, respectively.

From the discussion in [CKV15, §3], it follows that the inclusion $\widehat{R}_{C} \hookrightarrow \widehat{R}_{(C, I)}$ induces, by passing to the formal spectrum, a diagram

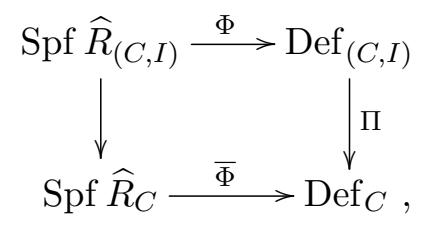


where $\Phi$ realizes $\widehat{R}_{(C, I)}$ as the mini-versal deformation ring of the functor $\operatorname{Def}_{(C, I)}$ (as discussed in the proof of Theorem 8.1) and $\bar{\Phi}$ realizes $\widehat{R}_{C}$ as the universal deformation ring of Def . $_{\text {. }}$ Moreover, $\Phi$ is $\operatorname{Aut}(C, I)$-equivariant (as discussed in the proof of Theorem 8.1), $\bar{\Phi}$ is clearly Aut $(C)$-equivariant (being an isomorphism of functors), and the two vertical maps in (8.21) are equivariant with respect to the group homomorphism $\operatorname{Aut}(C, I) \rightarrow \operatorname{Aut}(C)$.

Therefore, as an application of Luna's slice theorem (see $[\mathrm{CKV} 15, \S 6]$ ), we get a commutative diagram

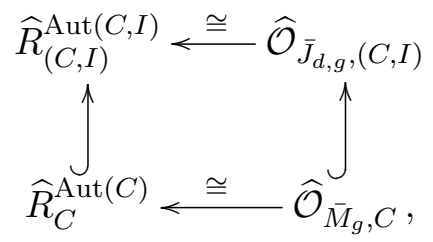

which concludes the proof.

Now, consider the graph $\Gamma=\Gamma_{(C, I)}$ obtained from the dual graph of $C$ by contracting the edges corresponding to nodes of $C$ where $I$ is locally free, as in Section 8.1. It follows from the above discussion that the inclusions $R_{C} \hookrightarrow R_{(C, I)}^{\mathrm{Aut}(I)} \hookrightarrow R_{(C, I)}$ are given, up to smooth factors, by the following inclusions of $k$-algebras (with the notation of Section 6 ):

$$
\begin{aligned}
k\left[T_{e}: e \in E(\Gamma)\right] \hookrightarrow U(\Gamma)=B(\Gamma)^{T_{\Gamma}} \hookrightarrow B(\Gamma)=k\left[X_{\vec{e}}: \vec{e} \in \vec{E}(\Gamma)\right], \\
T_{e} \mapsto X_{\vec{e}} \cdot X_{\overleftarrow{e}},
\end{aligned}
$$

where we have used that $X_{\vec{e}} \cdot X_{\overleftarrow{e}} \in B(\Gamma)$ is invariant under the action of $T_{\Gamma}$ given in (6.1). Therefore, we get the surjective morphism of varieties

$$
\operatorname{Spec} B(\Gamma)=\operatorname{Spec} k\left[X_{\vec{e}}: \vec{e} \in \vec{E}(\Gamma)\right] \stackrel{f}{\rightarrow} X_{\Gamma}=\operatorname{Spec} U(\Gamma) \stackrel{g}{\rightarrow} \operatorname{Spec} k\left[T_{e}: e \in E(\Gamma)\right] .
$$

These morphisms are toric morphisms of affine toric varieties, which can be described using toric geometry as follows. With the notation of Section 4, consider the injective linear maps

$$
\begin{aligned}
& \mathbb{R}\langle e\rangle_{e \in E(\Gamma)} \rightarrow \mathbb{H}_{1}(\Gamma, \mathbb{R})=\operatorname{ker} \mathbb{D} \rightarrow \mathbb{C}_{1}(\Gamma, \mathbb{R}) \\
& \sum_{e \in E(\Gamma)} a_{e} \cdot e \mapsto \sum_{e \in E(\Gamma)} a_{e}(\vec{e}+\overleftarrow{e}),
\end{aligned}
$$

which clearly preserve the integral lattices. By taking duals, we get the surjective lattice-preserving linear maps

$$
\mathbb{C}_{1}(\Gamma, \mathbb{R})^{\vee} \stackrel{l}{\rightarrow} \mathbb{H}_{1}(\Gamma, \mathbb{R})^{\vee} \stackrel{h}{\rightarrow} \mathbb{R}\left\langle e^{\vee}\right\rangle_{e \in E(\Gamma)}
$$

The three vectors spaces in (8.25) are endowed with standard scalar products that will be denoted with the same symbol ( , ) (see Sections 2.2 and 4). Inside the vector space $\mathbb{H}_{1}(\Gamma, \mathbb{R})^{\vee}$, we have the cone $\sigma:=\sigma_{\Gamma}^{\vee}$ introduced in Section 4. The rational polyhedral fan formed by $\sigma$ and all its faces corresponds to the toric variety $X_{\Gamma}$. Using Lemma 5.1(i), it follows that $\sigma$ is equal to

$$
\sigma=\operatorname{conv}\langle(\cdot, \vec{e})\rangle_{\vec{e} \in \vec{E}(\Gamma)},
$$

where conv denotes the convex hull. Set

$$
\begin{aligned}
& \widehat{\sigma}:=\operatorname{conv}\langle(\cdot, \vec{e})\rangle_{\vec{e} \in \vec{E}(\Gamma)} \subset \mathbb{C}_{1}(\Gamma, \mathbb{R})^{\vee} \\
& \widetilde{\sigma}:=\operatorname{conv}\langle(\cdot, e)\rangle_{e \in E(\Gamma)}=\mathbb{R}_{\geqslant 0}\left\langle e^{\vee}\right\rangle_{e \in E(\Gamma)} \subset \mathbb{R}\left\langle e^{\vee}\right\rangle_{e \in E(\Gamma)} .
\end{aligned}
$$




\section{S. Casalaina-Martin, J. L. Kass and F. Viviani}

Clearly, the cones $\widehat{\sigma}$ and $\widetilde{\sigma}$ gives rise to the toric varieties Spec $k\left[X_{\vec{e}}: \vec{e} \in \vec{E}(\Gamma)\right]$ and $\operatorname{Spec} k\left[T_{e}\right.$ : $e \in E(\Gamma)]$, respectively. Moreover, the lattice-preserving linear maps (8.25) are such that $l(\widehat{\sigma})=\sigma$ and $h(\sigma)=\widetilde{\sigma}$; hence, they induce morphisms $\operatorname{Spec} k\left[X_{\vec{e}}: \vec{e} \in \vec{E}(\Gamma)\right] \rightarrow X_{\Gamma} \rightarrow \operatorname{Spec} k\left[T_{e}: e \in\right.$ $E(\Gamma)]$ which are easily seen to coincide with the morphisms $f$ and $g$ of (8.24).

\subsection{Singularities of $\bar{M}_{g}$}

We recall the following result of Harris-Mumford and Ludwig.

Theorem 8.3 ([HM82, Theorem 2], [Lud07, Proposition 4.2.5, Corollary 4.2.6]). Let $g \geqslant 4$, $C \in \bar{M}_{g}$, and $\phi \in \operatorname{Aut}(C)$. Set $R_{C}$ to be a mini-versal space for $C$. If $\phi$ acts as a pseudoreflection on Spec $R_{C}$ or Spec $R_{C} /\langle\phi\rangle$ does not have canonical singularities, then the following hold:

(i) The curve $C$ has an elliptic tail $E \subset C$, that is, an irreducible subcurve of arithmetic genus one that meets the complementary subcurve $E^{c}:=\overline{C \backslash E}$ in one point $p$, and $\phi$ is an elliptic tail automorphism, that is, $\phi_{E^{c}}=\mathrm{id}_{E^{c}}$.

(ii) The restriction $\phi_{\mid E}$ is an automorphism of $E$, fixing $p$, with order $n=2,3,4$, or 6 . If $n=4$, then $E$ is smooth with $j$-invariant equal to 1728 , and if $n=3$ or 6 , then $E$ is smooth with $j$-invariant equal to 0 .

(iii) If $E$ is a singular elliptic curve, then $\phi_{\mid E}$ has order $n=2$ and is given as follows: Denote by $\nu: E^{\nu} \rightarrow E$ the normalization of $E$ and identify $E^{\nu}$ with $\mathbb{P}^{1}$ in such a way that $\nu^{-1}=\infty=(1,0)$ and $\nu^{-1}(q)=\{(1,1),(-1,1)\}$. Then $\phi_{\mid E}$ is induced by the involution of $\mathbb{P}^{1}$ sending $(x, y)$ into $(-x, y)$.

Moreover, let $g \geqslant 4$, let $C \in \bar{M}_{g}$ be a curve with an elliptic tail $E$, and let $\phi \in \operatorname{Aut}(C)$ be an elliptic tail automorphism (with respect to $E$ ). Let $\left\{t_{1}, \ldots, t_{3 g-3}\right\}$ be coordinates of $T \operatorname{Def}_{C}$ such that $t_{1}$ corresponds to the smoothing of $C$ at the node $p$ and $t_{2}$ corresponds, if $E$ is smooth, to a coordinate for $T_{(E, p)}\left(M_{1,1}\right)$ (corresponding to the $j$-invariant of $E$ ) and if $E$ is singular, to the smoothing of $C$ at $q$. Then the action of $\phi$ on $T \operatorname{Def}_{C}$ on these coordinates is given by the following matrix (depending on the choice of the primitive $n$th root of unity $\zeta$ ):

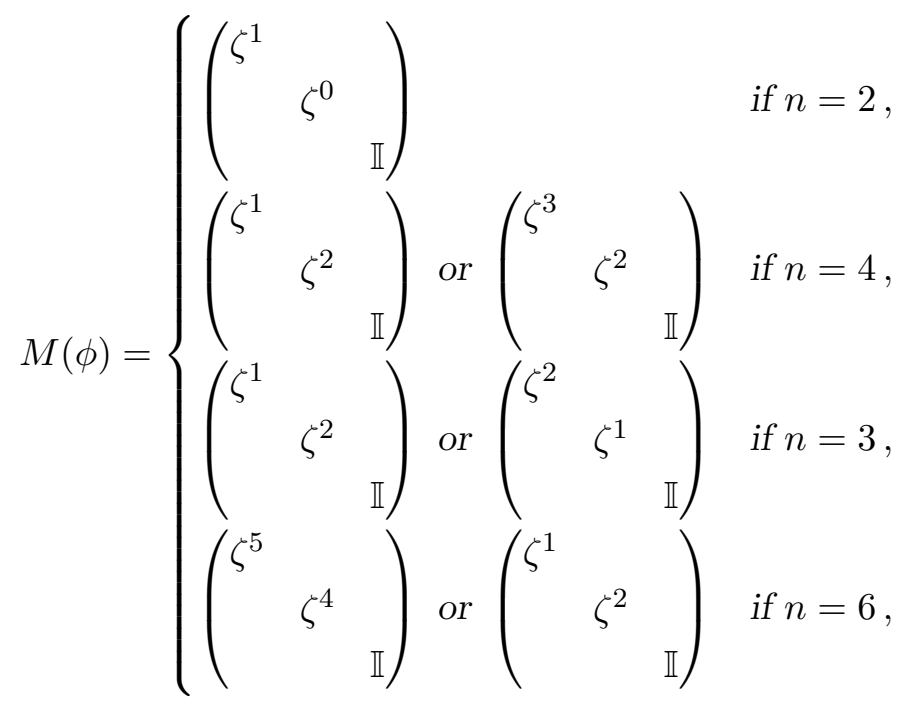

where $\mathbb{I}$ is the suitable identity matrix. 


\section{THE SINGULARITIES AND BIRATIONAL GEOMETRY}

\subsection{Singularities of $\bar{J}_{d, g}$}

The aim of this subsection is to prove that $\bar{J}_{d, g}$ has canonical singularities if $g \geqslant 4$ and $\operatorname{char}(k)=0$.

Theorem 8.4. Assume $\operatorname{char}(k)=0$ and $g \geqslant 4$. Then the compactified universal Jacobian $\bar{J}_{d, g}$ has canonical singularities for any $d \in \mathbb{Z}$.

Proof. Since the property of having canonical singularities is invariant under localization and completion (see, for example, [Mat02, Proposition 4-4-4]), it is enough to show, by Theorem 8.1, that the affine variety

$$
\operatorname{Spec}\left[\left(R_{(C, I)}\right)^{\operatorname{Aut}(C, I)}\right]=\operatorname{Spec}\left[\left(R_{(C, I)}^{\operatorname{Aut}(I)}\right)^{\operatorname{Stab}_{C}(I)}\right]=\operatorname{Spec}\left(R_{(C, I)}^{\operatorname{Aut}(I)}\right) / \operatorname{Stab}_{C}(I)
$$

has canonical singularities for every $(C, I) \in \bar{J}_{d, g}$.

Roughly speaking, the outline of the argument from this point on is as follows. We take the point $(C, I) \in \bar{J}_{d, g}$ and consider its image $C \in \bar{M}_{g}$. Then we break the argument into two parts: (1) $\bar{M}_{g}$ has canonical singularities near $C$ and (2) $\bar{M}_{g}$ does not have canonical singularities near $C$. In case (1), we use a generalization of the Reid-Tai criterion that can be applied to singular toric varieties (we review this generalization of Reid-Tai in the appendix), and we obtain that $\operatorname{Spec}\left(R_{(C, I)}^{\mathrm{Aut}(I)}\right) / \operatorname{Stab}_{C}(I)$ (and hence $\bar{J}_{d, g}$ ) has canonical singularities at $(C, I)$. In case (2), there is a short list due to Harris-Mumford of possible curves where $\bar{M}_{g}$ may fail to have canonical singularities (see Section 8.3). In these cases, it will turn out that $\operatorname{Spec}\left(R_{(C, I)}^{\operatorname{Aut}(I)}\right)$ is smooth. Thus, we can apply the usual Reid-Tai criterion. From the work of Harris-Mumford and Ludwig (see Section 8.3), one has an explicit description of the actions needed for the analysis. In the end, for case (2) the argument is very similar to that in [BFV12] and establishes that $\operatorname{Spec}\left(R_{(C, I)}^{\mathrm{Aut}(I)}\right) / \operatorname{Stab}_{C}(I)$ (and hence $\left.\bar{J}_{d, g}\right)$ also has canonical singularities at $(C, I)$ in this case. Technically, since we are able to focus on one automorphism of $(C, I)$ at a time, the argument is broken into somewhat finer pieces than just described, but this captures the main points.

We now proceed to implement this strategy.

To begin, a standard result (see Theorem A.11) says that $\operatorname{Spec}\left(R_{(C, I)}^{\operatorname{Aut}(I)}\right) / \operatorname{Stab}_{C}(I)$ has canonical singularities if and only if for every $\phi \in \operatorname{Stab}_{C}(I) \subseteq \operatorname{Aut}(C)$, the quotient

$$
\operatorname{Spec}\left(R_{(C, I)}^{\mathrm{Aut}(I)}\right) /\langle\phi\rangle
$$

has canonical singularities. Thus we proceed by considering the quotients $\operatorname{Spec}\left(R_{(C, I)}^{\operatorname{Aut}(I)}\right) /\langle\phi\rangle$.

Case 1. The automorphism $\phi \in \operatorname{Stab}_{C}(I)$ does not act as a pseudo-reflection on Spec $R_{C}$ and Spec $R_{C} /\langle\phi\rangle$ has canonical singularities.

We will show that $\operatorname{Spec}\left(R_{(C, I)}^{\mathrm{Aut}(I)}\right) /\langle\phi\rangle$ has canonical singularities. We will apply Lemma A.7, which is essentially a variation on the Reid-Tai criterion tailored to this setting, to the following morphism $\Psi$ induced by (8.18):

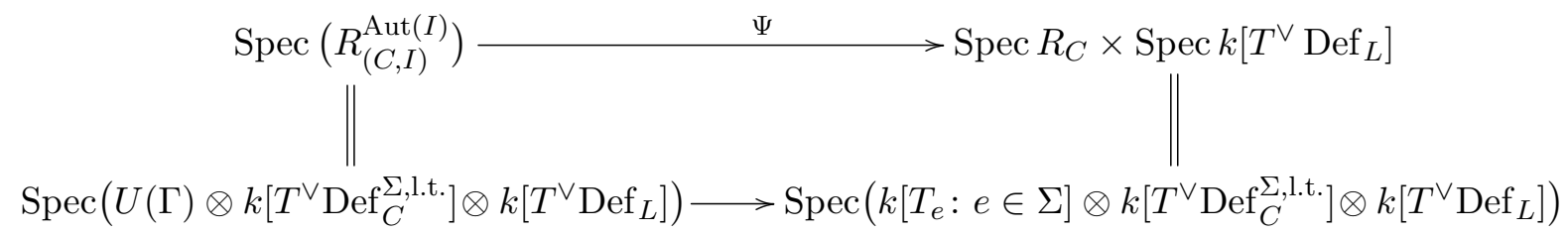

and the natural action of $\mathbb{Z}_{r}=\langle\phi\rangle$. The added factor of Spec $k\left[T^{\vee} \operatorname{Def}_{L}\right]$ on the right makes the computation more tractable. Let us check the hypotheses of Lemma A.7. 


\section{S. Casalaina-Martin, J. L. Kass and F. Viviani}

First of all, $\Psi$ is a toric morphism of affine toric varieties that acts as the identity on the last two factors Spec $k\left[T^{\vee} \operatorname{Def}_{C}^{\Sigma, \text { l.t. }}\right]$ and Spec $k\left[T^{\vee} \operatorname{Def}_{L}\right]$ and coincides with the map $g: X_{\Gamma}=$ $\operatorname{Spec} U(\Gamma) \rightarrow \operatorname{Spec} k\left[T_{e}: e \in E(\Gamma)\right]$ of (8.24) on the first factor. As explained in Section 8.2, the morphism $g$ is induced by the lattice-preserving linear map $h: \mathbb{H}_{1}(\Gamma, \mathbb{R})^{\vee} \rightarrow \mathbb{R}\left\langle e^{\vee}\right\rangle_{e \in E(\Gamma)}$ of $(8.25)$ which sends the cone $\sigma=\sigma_{\Gamma}^{\vee}$ associated with the toric variety $X_{\Gamma}$ to the cone $\widetilde{\sigma}$ corresponding to the toric variety Spec $k\left[T_{e}: e \in E(\Gamma)\right]$. By Lemma 5.1(iii), the extremal rays of $\sigma_{\Gamma}^{\vee}$ are given by $\langle(\cdot, \vec{e})\rangle:=\mathbb{R}_{\geqslant 0} \cdot \vec{e}^{\vee}$, as $\vec{e}$ varies among the oriented edges $\vec{E}(\Gamma)$ of $\Gamma$. As explained in Section 8.2, the linear map $h$ sends the extremal ray $\langle(\cdot, \vec{e})\rangle$ of the cone $\sigma=\sigma_{\Gamma}^{\vee}$ into the extremal ray $\langle(\cdot, e)\rangle$ of $\widetilde{\sigma}$, where $e \in E(\Gamma)$ is the (unoriented) edge underlying $\vec{e} \in \vec{E}(\Gamma)$. Furthermore, by Definition $(8.25)$, it follows that $h$ sends the primitive element $(\cdot, \vec{e})$ of the extremal ray $\langle(\cdot, \vec{e})\rangle$ (see Lemma 5.1(iv)) onto the primitive element $(\cdot, e)$ of the extremal ray $\langle(\cdot, e)\rangle$. This shows that hypotheses (i) and (ii) of Lemma A.7 are satisfied.

Now, consider the action of $\mathbb{Z}_{r}=\langle\phi\rangle \subset \operatorname{Stab}_{C}(I)$ on the domain and codomain of $\Psi$. The action preserves the decompositions of the domain and codomain, and the toric structure on the smooth factor $\operatorname{Spec}\left(k\left[T^{\vee} \operatorname{Def}_{C}^{\Sigma \text {,l.t. }}\right] \otimes k\left[T^{\vee} \operatorname{Def}_{L}\right]\right)$ is chosen via an eigenbasis for the action of $\phi$. By considering the modular interpretation of the other factors, we see that the two actions preserve the tori inside the domain and codomain, and, moreover, as observed in Section 8.2, the morphism $\Psi$ is $\mathbb{Z}_{r}$-equivariant. In addition, the toric variety $\operatorname{Spec} R_{C} \times \operatorname{Spec} k\left[T^{\vee} \operatorname{Def}_{L}\right]$ is smooth and $\mathbb{Z}_{r}$ acts on it without pseudo-reflections since, by assumption, $\phi$ already does not act as a pseudo-reflection on Spec $R_{C}$. This shows that the hypotheses (a) and (b) of Lemma A.7 are satisfied.

Finally, the quotient Spec $R_{C} /\langle\phi\rangle$ has canonical singularities by assumption. Using the ReidTai criterion (A.2) and the fact that $\phi$ does not act as a pseudo-reflection on Spec $R_{C}$, this is equivalent to the fact that the age of $\phi$ on $\operatorname{Spec} R_{C}$ with respect to any primitive $r$ th root of unity is greater than or equal to 1 . Of course, this remains true for the age of $\phi$ acting on the space Spec $R_{C} \times \operatorname{Spec} k\left[T^{\vee} \operatorname{Def}_{L}\right]$, which implies that $\left(\operatorname{Spec} R_{C} \times \operatorname{Spec} k\left[T^{\vee} \operatorname{Def}_{L}\right]\right) /\langle\phi\rangle$ has canonical singularities by the Reid-Tai criterion.

We can now apply Lemma A.7 in order to conclude that $\operatorname{Spec}\left(R_{(C, I)}^{\operatorname{Aut}(I)}\right) /\langle\phi\rangle$ has canonical singularities, which concludes the proof for case 1 .

Case 2. Either the automorphism $\phi \in \operatorname{Stab}_{C}(I) \subseteq \operatorname{Aut}(C)$ either acts as a pseudo-reflection on Spec $R_{C}$ or Spec $R_{C} /\langle\phi\rangle$ does not have canonical singularities.

The analysis we are going to perform in this case is similar to the analysis that was performed in $[\mathrm{BFV} 12, \S 4]$. However, there are two main differences: here, we use the Pandharipande [Pan96] modular interpretation of $\bar{J}_{d, g}$ instead of the Caporaso modular interpretation of $\bar{J}_{d, g}$ used in [Cap94]; moreover, we will not restrict ourselves to the stable locus, contrary to [Cap94].

To begin, according to the results of Harris-Mumford and Ludwig (see Theorem 8.3), case 2 can occur only if $C$ has an elliptic tail $E \subset C$, that is, a connected subcurve of arithmetic genus one which meets the complementary subcurve $E^{c}:=\overline{C \backslash E}$ in one point $p$, and $\phi$ is an elliptic tail automorphism, that is, $\phi_{E_{E^{c}}}=\mathrm{id}_{E^{c}}$. We now consider two sub-cases:

Case 2-I: The sheaf $I$ is not locally free at $p$.

Case 2-II: The sheaf $I$ is locally free at $p$. 


\section{THE SINGULARITIES AND BIRATIONAL GEOMETRY}

Note that in either case, if $E$ is a rational elliptic tail with one node $q$, then $I$ could be locally free, or not, at $q$.

Now, consider the ring $R_{(C, I)}^{\mathrm{Aut}(I)}$ as in (8.8). As usual, denote by $\Gamma=\Gamma_{(C, I)}$ the graph obtained from the dual graph of $C$ by contracting all the edges corresponding to nodes of $C$ where $I$ is locally free. Moreover, denote by $\Gamma_{E}$ and $\Gamma_{E^{c}}$ the graphs obtained from the dual graphs of $E$ and $E^{c}$ by contracting all the edges corresponding to the nodes of $E$ and $E^{c}$, respectively, where $I$ is locally free.

In case 2-II, the graph $\Gamma$ is obtained by joining the graphs $\Gamma_{E}$ and $\Gamma_{E^{c}}$ along a common vertex, and in case 2-I by means of a separating edge corresponding to the node $p$. Therefore, from the explicit presentation of $U(\Gamma) \cong D(\Gamma)$ given in Theorem 4.6 (see also Lemma 5.3), it follows that

$$
U(\Gamma)= \begin{cases}U\left(\Gamma_{E^{c}}\right) \otimes_{k} U\left(\Gamma_{E}\right) \otimes_{k} k\left[T_{p}\right] & (\text { case } 2-\mathrm{I}) \\ U\left(\Gamma_{E^{c}}\right) \otimes_{k} U\left(\Gamma_{E}\right) & (\text { case 2-II) } .\end{cases}
$$

The graph $\Gamma_{E}$ consists of a vertex with a loop if $E$ is a rational elliptic tail with one node $q$ and $I$ is not locally free at $q$; otherwise, $\Gamma_{E}$ has one vertex and no edges. Therefore, using Theorem 6.2 (and, say, Example 3.2), we easily compute

$$
U\left(\Gamma_{E}\right)=D\left(\Gamma_{E}\right)= \begin{cases}k\left[X_{q}, Y_{q}\right] & \text { if } E \text { has a node } q \text { and } I \text { is not locally free at } q, \\ k & \text { otherwise }\end{cases}
$$

Now, consider the automorphism $\phi \in \operatorname{Stab}_{C}(I)$. Clearly, $\phi$ acts on $U(\Gamma)$ by preserving the decomposition (8.28), and, moreover, since $\phi_{E^{c}}=\mathrm{id}_{E^{c}}$ by assumption, $\phi$ acts trivially on $U\left(\Gamma_{E^{c}}\right)$. Therefore, we have

$\operatorname{Spec}\left(R_{(C, I)}^{\operatorname{Aut}(I)}\right) /\langle\phi\rangle= \begin{cases}\operatorname{Spec} U\left(\Gamma_{E^{c}}\right) \times \operatorname{Spec}\left(U\left(\Gamma_{E}\right) \otimes_{k} k\left[T_{p}\right] \otimes_{k} k\left[T^{\vee} \operatorname{Def}_{(C, I)}^{\text {l.t. }}\right]\right) /\langle\phi\rangle & \text { (case 2-I }), \\ \operatorname{Spec} U\left(\Gamma_{E^{c}}\right) \times \operatorname{Spec}\left(U\left(\Gamma_{E}\right) \otimes_{k} k\left[T^{\vee} \operatorname{Def}_{(C, I)}^{\text {l.t. }}\right]\right) /\langle\phi\rangle & \text { (case 2-II). }\end{cases}$

Since Spec $U\left(\Gamma_{E^{c}}\right)$ has canonical (and even terminal) singularities by Theorem 5.5 , it is enough to prove that $\operatorname{Spec}\left(U\left(\Gamma_{E}\right) \otimes_{k} k\left[T_{p}\right] \otimes_{k} k\left[T^{\vee} \operatorname{Def}_{(C, I)}^{\text {l.t. }}\right]\right) /\langle\phi\rangle$ and $\operatorname{Spec}\left(U\left(\Gamma_{E}\right) \otimes_{k} k\left[T^{\vee} \operatorname{Def}_{(C, I)}^{\text {l.t. }}\right]\right) /\langle\phi\rangle$ have canonical singularities. Taking into account (8.29), we see that in both cases we are dealing with finite quotient singularities, so that we can apply the classical Reid-Tai criterion (see Theorem A.1) to check canonicity.

Before applying the criterion, recall from (8.17) the splitting

$$
k\left[T^{\vee} \operatorname{Def}_{(C, I)}^{\text {l.t. }}\right] \cong k\left[T^{\vee} \operatorname{Def}_{C}^{\Sigma, \text { l.t. }}\right] \otimes_{k} k\left[T^{\vee} \operatorname{Def}_{L}\right],
$$

where $L$ is the unique line bundle on the partial normalization $g: C_{\Sigma} \rightarrow C$ of $C$ at the nodes $\Sigma=\Sigma_{(C, I)}$ with the property that $g_{*}(L)=I$. We now want to choose a suitable basis of the vector space

$$
V:= \begin{cases}T U\left(\Gamma_{E}\right) \oplus T k\left[T_{p}\right] \oplus T \operatorname{Def}_{C}^{\Sigma, \text { l.t. }} \oplus T \operatorname{Def}_{L} & (\text { case 2-I }), \\ T U\left(\Gamma_{E}\right) \oplus T \operatorname{Def}_{C}^{\Sigma, \text { l.t. }} \oplus T \operatorname{Def}_{L} & \text { (case 2-II), }\end{cases}
$$

and compute the matrix $R(\phi)$ of $\phi$ in terms of the chosen basis.

First, observe that in both case 2-I and case 2-II, the upper-left $2 \times 2$ submatrix of $M(\phi)$ from (8.26) appears as a block factor of the matrix $R(\phi)$. Indeed, in case 2-I, we can choose the coordinate $t_{1}$ of $T \operatorname{Def}_{C}$ corresponding to the smoothing of $C$ at the node $p$ as a coordinate of $T k\left[T_{p}\right]$, and in case 2 -II, we can choose $t_{1}$ as one of the coordinates of $T \operatorname{Def}_{C}^{\Sigma \text {,l.t. }}$. Moreover, if 


\section{S. Casalaina-Martin, J. L. Kass and F. Viviani}

$n>2$ (which implies that $E$ is smooth), then we can choose the coordinate $t_{2}$ of $T \operatorname{Def}_{C}$ coming from $T_{(E, p)}\left(M_{1,1}\right)$ as one of the coordinates of $T \operatorname{Def}_{C}^{\Sigma, \text { l.t. }}$.

We now focus our attention on the action of $\phi$ on $T \operatorname{Def}_{L}$. Denote by $E_{\Sigma}^{c}$ and $E_{\Sigma}$ the normalizations of $E^{c}$ and $E$, respectively, at the nodes belonging to $\Sigma$. The curve $C_{\Sigma}$ is the disjoint union of $E_{\Sigma}^{c}$ and $E_{\Sigma}$ in case 2-I, while it is obtained by joined $E_{\Sigma}^{c}$ and $E_{\Sigma}$ at the separating point $p$ in case 2-II. In any case, $L$ is completely determined by its restrictions $L_{\mid E_{\Sigma}^{c}}$ and $L_{\mid E_{\Sigma}}$, and, moreover, we have a decomposition

$$
T \operatorname{Def}_{L}=T \operatorname{Def}_{L_{\mid E_{\Sigma}}} \oplus T \operatorname{Def}_{L_{\mid E_{\Sigma}^{c}}} .
$$

Since $\phi_{\mid E^{c}}=\operatorname{id}_{E^{c}}$ by assumption, $\phi$ acts trivially on $T \operatorname{Def}_{L_{\mid E_{\Sigma}^{c}}}$.

At this point, we have established what we need from the breakdown of case 2 into cases 2-I and 2-II. In short, in all of case 2, the upper-left $2 \times 2$ submatrix of $M(\phi)$ from (8.26) will appear as a block factor of the matrix $R(\phi)$, and the action on $T \operatorname{Def}_{L}$ is determined by the action on $T \operatorname{Def}_{L_{\mid E_{\Sigma}}} \cong T_{L_{\mid E_{\Sigma}}}\left(\operatorname{Pic}\left(E_{\Sigma}\right)\right)$.

Let us now examine the action of $\phi$ on $T \operatorname{Def}_{L_{\mid E_{\Sigma}}}$. For this we consider three new sub-cases of case 2 :

Case 2-i: $E$ is smooth.

Case 2-ii: $E$ is a rational elliptic curve with one node $q$ and $I$ is locally free at $q$.

Case 2-iii: $E$ is a rational elliptic curve with one node $q$ and $I$ is not locally free at $q$.

We now proceed with a case by case analysis.

Case 2-i. We are assuming that $E$ is smooth. Consequently, $E_{\Sigma}=E$ and $L_{\mid E_{\Sigma}}=I_{E} \in$ $\operatorname{Pic}^{d_{E}}(E)$. We can identify $E$ with $\operatorname{Pic}^{d_{E}}(E)$, sending $r \in E$ into $\mathcal{O}_{E}\left(r+\left(d_{E}-1\right) p\right) \in \operatorname{Pic}^{d_{E}}(E)$. Since $\phi$ acts on $\operatorname{Pic}^{d_{E}}(E)$ via pull-back, if the action of $\phi$ on $T_{p}(E)$ is given by the multiplication by a root of unity $\zeta$, then the action of $\phi$ on $T_{I_{E}}\left(\operatorname{Pic}^{d_{E}}(E)\right)$ is given by the multiplication by $\zeta^{-1}$. In other words, if the primitive $n$th root of unity $\zeta$ is chosen for the matrix $M(\phi)$ from $(8.26)$, then here, the action is given by the primitive $n$th root of unity $\zeta^{-1}$. Therefore, the matrix $N(\phi)$ of $\phi$ with respect to the decomposition (8.32) is equal to (with respect to the same choice of the primitive $n$th root of unity $\zeta$ as in the above matrix $M(\phi))$

$$
N(\phi)= \begin{cases}\left(\begin{array}{ll}
\zeta^{1} & \\
& \mathbb{I}
\end{array}\right) & \text { if } n=2, \\
\left(\begin{array}{ll}
\zeta^{3} & \\
& \mathbb{I}
\end{array}\right) \text { or }\left(\begin{array}{ll}
\zeta^{1} & \\
& \mathbb{I}
\end{array}\right) & \text { if } n=4, \\
\left(\begin{array}{ll}
\zeta^{2} & \\
& \mathbb{I}
\end{array}\right) \text { or }\left(\begin{array}{ll}
\zeta^{1} & \\
& \mathbb{I}
\end{array}\right) & \text { if } n=3, \\
\left(\begin{array}{ll}
\zeta^{1} & \\
& \mathbb{I}
\end{array}\right) \text { or }\left(\begin{array}{ll}
\zeta^{5} & \\
& \mathbb{I}
\end{array}\right) & \text { if } n=6,\end{cases}
$$

where $\mathbb{I}$ is the suitable identity matrix. Note that the first matrix in each row above corresponds to the first matrix in the corresponding row of (8.26). The matrix $R(\phi)$ describing the action of $\phi$ on the vector space $V$ (8.31) contains the upper-left $2 \times 2$ submatrix of $M(\phi)$ from (8.26) and the upper-left $1 \times 1$ submatrix of $N(\phi)$ from (8.33) as block fac- 


\section{THE SINGULARITIES AND BIRATIONAL GEOMETRY}

tors. An easy inspection of the matrices $M(\phi)$ and $N(\phi)$ reveals that the condition (A.2) of the Reid-Tai criterion is satisfied, which shows that $V /\langle\phi\rangle$ has canonical singularities, as we wanted.

Case 2-ii. In this case, we are assuming that $E$ is a rational elliptic curve with one node $q$ and that $I$ is locally free at $q$. Then, also in this case, $E_{\Sigma}=E$ and $L_{\mid E_{\Sigma}}=I_{E} \in \operatorname{Pic}^{d_{E}}(E)$. Moreover, we have $\operatorname{Pic}^{d_{E}}(E) \cong \mathbb{G}_{m}$. Explicitly, if we consider the normalization morphism $\nu: E^{\nu} \cong \mathbb{P}^{1} \rightarrow E$ and let $\nu^{-1}(q)=\{u, v\}$, then any $\lambda \in \mathbb{G}_{m}$ determines a unique line bundle $L_{\lambda} \in \operatorname{Pic}^{d_{E}}(E)$ whose local sections are the local sections $s$ of $\mathcal{O}_{\mathbb{P}^{1}}\left(d_{E}\right)$ such that $s(u)=\lambda s(v)$. Since, as observed before, $\phi_{\mid E}$ is induced by an involution of $E^{\nu}$ that exchanges $u$ and $v$, clearly $\phi$ will send $L_{\lambda}$ into $L_{\lambda^{-1}}$. This implies that the action of $\phi$ on $T_{I_{E}}\left(\mathrm{Pic}^{d_{E}}(E)\right)$ is given by multiplication by -1 ; hence, also in this case, the matrix $N(\phi)$ is given by (8.33) with $n=2$.

Therefore, the matrix $R(\phi)$ describing the action of $\phi$ on the vector space $V$ contains the upper-left $2 \times 2$ submatrix $M(\phi)$ from (8.26) and the upper-left $1 \times 1$ submatrix of $N(\phi)$ from $(8.33)$ as block factors, and we conclude as in the previous case that $V /\langle\phi\rangle$ has canonical singularities, as we wanted.

Case 2-iii. In this case, $E$ is a rational elliptic tail with one node $q$, and $I$ is not locally free at $q$. Observe that in this case $E_{\Sigma}=\mathbb{P}^{1}$, so that $T \operatorname{Def}_{L_{\mid E_{\Sigma}}}=0$ and hence the action of $\phi$ on $T \operatorname{Def}_{L}$ is trivial. To proceed in this case, we consider instead the action of $\phi$ on $T U\left(\Gamma_{E}\right)$, which is a two-dimensional $k$-vector space since $U\left(\Gamma_{E}\right)=k\left[X_{q}, Y_{q}\right]$ by (8.29). Geometrically, the variables $X_{q}$ and $Y_{q}$ correspond to deforming the sheaf $I$ at $q$ along the two branches of $q$ (see [CKV15, §3] for more details). Since, as observed before, $\phi_{\mid E}$ is induced by an involution of the normalization $\nu: E^{\nu} \rightarrow E$ that exchanges the two branches above $q$, the morphism $\phi$ acts on $U\left(\Gamma_{E}\right)=k\left[X_{q}, Y_{q}\right]$ by exchanging $X_{q}$ with $Y_{q}$. Therefore, we can diagonalize the action of $\phi$ on $T U\left(\Gamma_{E}\right)=\left\langle X_{q}^{\vee}, Y_{q}^{\vee}\right\rangle$ by choosing the basis $\left\{X_{q}^{\vee}-\right.$ $\left.Y_{q}^{\vee}, X_{q}^{\vee}+Y_{q}^{\vee}\right\}$ in such a way that the matrix $P(\phi)$ describing the action of $\phi$ is equal to

$$
P(\phi)=\left(\begin{array}{cc}
-1 & 0 \\
0 & 1
\end{array}\right)
$$

Therefore, since the matrix $R(\phi)$ describing the action of $\phi$ on the vector space $V$ contains the upper-left $2 \times 2$ submatrix of $M(\phi)$ from (8.26) with $n=2$ and the matrix $P(\phi)$ of (8.34) as block factors, an easy inspection of the matrices $M(\phi)$ and $P(\phi)$ reveals that the condition (A.2) of the Reid-Tai criterion is also satisfied in this case, which shows that $V /\langle\phi\rangle$ has canonical singularities, as we wanted.

Theorem 8.4 was proved by Bini-Fontanari-Viviani in [BFV12] under the assumption that $\operatorname{gcd}(d+1-g, 2 g-2)=1$, which is exactly the numerical condition on $d$ and $g$ that guarantees that $\bar{J}_{d, g}$ has finite quotient singularities. When this happens, one can prove Theorem 8.4 by a direct application of the Reid-Tai criterion (see [BFV12, Theorem 4.8]).

Remark 8.5. It follows from Theorem 8.4 that $\bar{J}_{d, g}$ is $\mathbb{Q}$-Gorenstein. Indeed, more is true: Fontanari showed in [Fon05] that $\bar{J}_{d, g}$ is $\mathbb{Q}$-factorial.

We end this subsection with a description of the locus where $\bar{J}_{d, g}$ has finite quotient singularities or is smooth. 


\section{S. Casalaina-Martin, J. L. Kass and F. Viviani}

Proposition 8.6. Let $(C, I) \in \bar{J}_{d, g}$, and assume that Aut $(C)$ does not contain elements of order equal to $p=\operatorname{char}(k)$. Then, the following hold:

(i) The compactified universal Jacobian $\bar{J}_{d, g}$ has finite quotient singularities at $(C, I)$ if and only if $\Gamma_{(C, I)}$ is tree-like, that is, it becomes a tree after removing all the loops around its vertices.

(ii) If $g \geqslant 4$, then $\bar{J}_{d, g}$ is smooth at $(C, I)$ if and only if $\Gamma_{(C, I)}$ is tree-like and $\operatorname{Stab}_{C}(I)=\{\operatorname{Id}\}$.

Proof. (i) Using the presentation of the complete local ring of $\bar{J}_{d, g}$ at $(C, I)$ given in Theorem 8.1, it is clear that $\bar{J}_{d, g}$ has finite quotient singularities at $(C, I)$ if and only if $X_{\Gamma}=\operatorname{Spec} U(\Gamma)$ has finite quotient singularities, where $\Gamma=\Gamma_{(C, I)}$. Proposition 5.7 says that this is the case if and only if $\Gamma$ is tree-like, concluding the proof.

(ii) By Theorem 8.1, the smoothness of $\bar{J}_{d, g}$ at $(C, I)$ is equivalent to the smoothness of the quotient $\operatorname{Spec}\left(R_{(C, I)}^{\operatorname{Aut}(I)}\right) / \operatorname{Stab}_{C}(I)$. By part $((\mathrm{i}))$, we must have that $\Gamma=\Gamma_{(C, I)}$ is tree-like. In this case, $\operatorname{Spec}\left(R_{(C, I)}^{\operatorname{Aut}(I)}\right)=X_{\Gamma} \times \operatorname{Spec} k\left[T^{\vee} \operatorname{Def}_{(C, I)}^{\text {l.t. }}\right]$ is smooth by Proposition 5.7.

Claim 8.7. The finite group $\operatorname{Stab}_{C}(I)$ acts on $\operatorname{Spec}\left(R_{(C, I)}^{\mathrm{Aut}(I)}\right)$ without pseudo-reflections.

Proof. Indeed, consider the morphism $\operatorname{Spec}\left(R_{(C, I)}^{\mathrm{Aut}(I)}\right) \rightarrow \operatorname{Spec} R_{C}$ of smooth varieties. If $1 \neq$ $\phi \in \operatorname{Stab}_{C}(I)$ acts as a pseudo-reflection on $\operatorname{Spec}\left(R_{(C, I)}^{\operatorname{Aut}(I)}\right)$, then $\phi$ acts as a pseudo-reflection on Spec $R_{C}$. It is well known that this happens if and only if $C$ has an elliptic tail $E$ and $\phi$ is the elliptic tail involution, that is, $\phi_{\mid E^{c}}=\operatorname{id}_{E^{c}}$ and $\phi_{E}$ is the elliptic involution on $E$ (see Theorem 8.3). This situation is a special case of the situation we dealt with in case II of the proof of Theorem 8.4, where, in particular, we verified that the age of $\phi$ (with respect to its action on $\operatorname{Spec}\left(R_{(C, I)}^{\operatorname{Aut}(I)}\right)$ and any primitive root of unity) is at least one. This easily implies that $\phi$ is not a pseudo-reflection because clearly any non-trivial pseudo-reflection has age less than one since it has a unique eigenvalue different from one.

Proof of Proposition 8.6, continued. Using the claim, we conclude the proof using a classical result of Prill [Pri67], which says that for a finite group $G$ acting on a smooth variety $X$ without pseudo-reflections, the quotient $X / G$ is smooth if and only if $G$ is the trivial group.

Part ((ii)) of Proposition 8.6 generalizes [BFV12, Proposition 4.7], where the statement is proved under the assumption that $(C, I)$ belongs to the stable locus of $\bar{J}_{d, g}$, that is, $I$ is stable with respect to $\omega_{C}$.

Remark 8.8. From Proposition 8.6((i)), it follows that the locus where $\bar{J}_{d, g}$ has finite quotient singularities is, in general, strictly bigger than

- the stable locus of $\bar{J}_{d, g}$, which coincides with the locus of points $(C, I)$ such that $\operatorname{Aut}(I)=$ $\mathbb{G}_{m}$ or, equivalently, $\Gamma_{(C, I)}$ has a unique vertex,

- the locus where the fibers of the morphism $\bar{J}_{d, g} \rightarrow \overline{\mathrm{M}}_{g}$ have finite quotient singularities, which coincides with the locus of points $(C, I)$ where $I$ fails to be locally free only at separating nodes of $C$ or, equivalently, where $\Gamma_{(C, I)}$ is a tree (see [CKV15, Theorem B]).

\subsection{Birational geometry of $\bar{J}_{d, g}$}

The Kodaira dimension of $J_{d, g}$ was computed by Bini-Fontanari-Viviani in [BFV12] under the numerical assumption that $\operatorname{gcd}(d+1-g, 2 g-2)=1$ (or $g \geqslant 22$; see Remark 8.10). However, the only place where the authors of [BFV12] need the hypothesis that $\operatorname{gcd}(d+1-g, 2 g-2)=1$ is to 


\section{THE SINGULARITIES AND BIRATIONAL GEOMETRY}

establish that $\bar{J}_{d, g}$ has canonical singularities, as they observe in the discussion following [BFV12, Theorem 1.4]. Therefore, as a corollary of [BFV12] and Theorem 8.4, we obtain the following result describing the Kodaira dimension of $J_{d, g}$.

Corollary 8.9. Assume $\operatorname{char}(k)=0$. The Kodaira dimension of the universal Jacobian $J_{d, g}$ is given by

$$
\kappa\left(J_{d, g}\right)= \begin{cases}-\infty & \text { if } g \leqslant 9 \\ 0 & \text { if } g=10 \\ 19 & \text { if } g=11 \\ 3 g-3 & \text { if } g \geqslant 12\end{cases}
$$

Proof. We sketch the proof for the convenience of the reader. Verra has shown that $J_{d, g}$ is unirational for $g \leqslant 9$ [Ver05, Theorem 1.2]. So let us consider the case $g \geqslant 10$. Let $\pi: \bar{J}_{d, g} \rightarrow \bar{M}_{g}$ be the natural forgetful map. Using the Grothendieck-Riemann-Roch theorem, it is shown in [BFV12, Theorem 1.5] that for $g \geqslant 4$, we have $K_{\bar{J}_{d, g}}=\pi^{*}(14 \lambda-2 \delta)\left(=\pi^{*} K_{\bar{M}_{g}}+\pi^{*} \lambda\right.$, agreeing with the naive computation over $M_{g}^{\circ}$ ). As $\pi$ has connected fibers, the Iitaka dimensions of $K_{\bar{J}_{d, g}}$ and $14 \lambda-2 \delta$ are the same. The Iitaka dimension of $14 \lambda-2 \delta$ is by now well known: $\kappa(14 \lambda-2 \delta)=0$ if $g=10, \kappa(14 \lambda-2 \delta)=19$ if $g=11$, and $\kappa(14 \lambda-2 \delta)=3 g-3$ if $g \geqslant 12$. (Recall that for $g \geqslant 13$, work of Eisenbud, Harris, and Mumford [HM82, EH87] shows that the slope of $\bar{M}_{g}$ satisfies $s\left(\bar{M}_{g}\right)<7$, and recent work of Cotterill [Cot12] shows that the same holds for $g=12$. For $g=10,11$, work of Tan [Tan98] and Farkas-Popa [FP05] shows that $s\left(\bar{M}_{g}\right)=7$; in these cases, $\kappa(14 \lambda-2 \delta)$ is worked out directly in $[\mathrm{BFV} 12, \S 6]$.) Finally, since in Theorem 8.4 we have shown that $\bar{J}_{d, g}$ has canonical singularities, we can conclude that $\kappa\left(\bar{J}_{d, g}\right)=\kappa\left(K_{\bar{J}_{d, g}}\right)$, completing the proof.

Remark 8.10. From general results of Ueno [Uen75, Theorem 6.12] and Kawamata [Kaw85, Corollary 1.2], using the fact that the Kodaira dimension of an abelian variety is zero, one obtains the estimate on the Kodaira dimension: $\kappa\left(\bar{M}_{g}\right) \leqslant \kappa\left(\bar{J}_{d, q}\right) \leqslant \operatorname{dim} \bar{M}_{g}$. By virtue of the results of Harris-Mumford, Eisenbud-Harris, and Farkas that $\bar{M}_{g}$ is of general type for $g=22$ and for $g \geqslant 24$, one obtains immediately that $\kappa\left(\bar{J}_{d, g}\right)=\kappa\left(\bar{M}_{g}\right)=3 g-3$ for $g$ in this range.

Remark 8.11. Since the generic fiber of $\pi: \bar{J}_{d, g} \rightarrow \bar{M}_{g}$ has trivial canonical bundle, it is interesting to compare the Kodaira dimensions of the two spaces. For the convenience of the reader, in the table below we compile the current state of the art on the Kodaira dimension of $\bar{M}_{g}$ (we refer the reader to Farkas [Far09] for references) and compare it with the Kodaira dimension of $\bar{J}_{d, g}$.

\begin{tabular}{c|c|c|c|c|c|c|c|c|c|c} 
& $g \leqslant 7$ & 8 & 9 & 10 & 11 & $12 \leqslant g \leqslant 16$ & $17 \leqslant g \leqslant 21$ & 22 & 23 & $24 \leqslant g$ \\
\hline$\kappa\left(\bar{M}_{g}\right)$ & $-\infty$ & $-\infty$ & $-\infty$ & $-\infty$ & $-\infty$ & $-\infty$ & unknown & $3 g-3$ & $\geqslant 2$ & $3 g-3$ \\
$\kappa\left(\bar{J}_{d, g}\right)$ & $-\infty$ & $-\infty$ & $-\infty$ & 0 & 19 & $3 g-3$ & $3 g-3$ & $3 g-3$ & $3 g-3$ & $3 g-3$ \\
\hline \hline$\kappa\left(\mathcal{S}_{g}^{-}\right)$ & $-\infty$ & $-\infty$ & $-\infty$ & $-\infty$ & $-\infty$ & $3 g-3$ & $3 g-3$ & $3 g-3$ & $3 g-3$ & $3 g-3$ \\
$\kappa\left(\mathcal{S}_{g}^{+}\right)$ & $-\infty$ & 0 & $3 g-3$ & $3 g-3$ & $3 g-3$ & $3 g-3$ & $3 g-3$ & $3 g-3$ & $3 g-3$ & $3 g-3$
\end{tabular}

Remark 8.12. In recent work, Farkas-Verra [Far10, FV12, Far12, FV14] have computed the Kodaira dimension of the moduli space of spin curves, that is, the moduli space of pairs consisting of a curve together with a theta characteristic. For each $g \geqslant 2$, the space has two components, $\mathcal{S}_{g}^{+}$and $\mathcal{S}_{g}^{-}$, corresponding to the even and odd theta characteristics. Since these sit inside $J_{g-1, g}$ and are finite over $M_{g}$, we find it interesting to compare the Kodaira dimensions of these spaces; 


\section{S. Casalaina-Martin, J. L. Kass and F. Viviani}

see (8.35). It turns out, for instance, that both $\bar{J}_{d, g}$ and $\mathcal{S}_{g}^{-}$attain "maximal" Kodaira dimension at $g=12$.

In [BFV12, Propositions 6.3 and 6.5], the Iitaka fibration of the canonical class $K_{\bar{J}_{d, g}}$ is established for $g \geqslant 10$. This provides the Iitaka fibration for $J_{d, g}$ under the additional hypothesis that $\bar{J}_{d, g}$ has canonical singularities. Consequently, [BFV12] gives the Iitaka fibration for $J_{d, g}$ assuming that $\operatorname{gcd}(d+1-g, 2 g-2)=1$ (and also for $g \geqslant 22$ using a different argument; see [BFV12, Proposition 3.2]). As a consequence of Theorem 8.4, we obtain the following result, generalizing those of [BFV12].

Corollary 8.13. For $g \geqslant 10$, the Iitaka fibration of $J_{d, g}$ is given as follows:

(i) For $g \geqslant 12$, the Iitaka fibration is the forgetful morphism $\pi: \bar{J}_{d, g} \rightarrow \bar{M}_{g}$.

(ii) For $g=11$, the Iitaka fibration is the rational map $\bar{J}_{d, 11} \rightarrow \mathcal{F}_{11}$, where $\mathcal{F}_{g}$ is the moduli space of $K 3$ surfaces with polarization of degree $2 g-2$, and the rational map takes a general pair $(C, L)$ to the pair $\left(S, \mathcal{O}_{S}(C)\right)$, where $S$ is the unique $K 3$ containing $C$ (see [Muk96]).

(iii) For $g=10$, the Iitaka fibration is the structure morphism $\bar{J}_{d, 10} \rightarrow$ Spec $k$.

Proof. We sketch the proof for the convenience of the reader. For $g \geqslant 12$, this follows from Theorem 8.4 and [Uen75, Theorem 6.11]. Indeed, let $\widetilde{M}_{g}$ be a resolution of singularities of $\bar{M}_{g}$, and let $\tilde{J}_{d, g}$ be a resolution of singularities of the fiber product $\bar{J}_{d, g} \times \bar{M}_{g} \widetilde{M}_{g}$. Then the morphism $\tilde{\pi}: \tilde{J}_{d, g} \rightarrow \widetilde{M}_{g}$ of smooth projective varieties is an algebraic fiber space such that $\operatorname{dim} \widetilde{M}_{g}=\kappa\left(\tilde{J}_{d, g}\right)$ and the generic fiber $\tilde{\pi}^{-1}(C)=J^{d} C$ is smooth and irreducible of Kodaira dimension zero. The same argument works for $g=10$, using a desingularization $\tilde{J}_{d, 10}$ of $\bar{J}_{d, 10}$. For $g=11$, we refer the reader to [BFV12, Proposition 6.5], where it is shown that the rational map $\bar{J}_{d, 11}-\rightarrow \mathcal{F}_{11}$ is the Iitaka fibration for $K_{\bar{J}_{d, 11}}$. Since $\bar{J}_{d, g}$ has canonical singularities by Theorem 8.4 , it follows that this rational map is the Iitaka fibration for $J_{d, 11}$.

In the last section of [BFV12], the authors investigate the birational maps among the different universal Jacobians $J_{d, g}$ as $d$ varies. Using Theorem 8.4, we can relax their hypothesis (see the discussion at the end of $[\mathrm{BFV} 12, \S 7])$.

Corollary 8.14. Assume $\operatorname{char}(k)=0$ and $g \geqslant 12$. If $\eta: J_{d, g} \rightarrow J_{d^{\prime}, g}$ is a birational map, then $d^{\prime}= \pm d+n(2 g-2)$ and $\eta$ is given by the map sending $(C, L) \in J_{d, g}$ into $\left(C, L^{ \pm 1} \otimes \omega_{C}^{n}\right) \in J_{d^{\prime}, g}$. In particular, the following hold:

(i) The Jacobian $J_{d, g}$ is birational to $J_{d^{\prime}, g}$ if and only if $d^{\prime} \equiv \pm d \bmod 2 g-2$.

(ii) The group $\operatorname{Bir}\left(J_{d, g}\right)$ of birational automorphisms of $J_{d, g}$ is given by

$$
\operatorname{Bir}\left(J_{d, g}\right)= \begin{cases}\mathbb{Z} / 2 \mathbb{Z} & \text { if } d=n(g-1) \text { for some } n \in \mathbb{Z}, \\ \{\mathrm{Id}\} & \text { otherwise }\end{cases}
$$

Moreover, if $d=n(g-1)$ for some $n \in \mathbb{Z}$, then the generator of $\operatorname{Bir}\left(J_{d, g}\right)$ is the birational automorphism sending $(C, L)$ into $\left(C, L^{-1} \otimes \omega_{C}^{n}\right)$.

Proof. We sketch the proof for the convenience of the reader. As established in Corollary 8.13, for $g \geqslant 12$, the morphism $\pi: \bar{J}_{d, g} \rightarrow \bar{M}_{g}$ is the Iitaka fibration of $J_{d, g}$. It follows that any birational automorphism $\eta: \bar{J}_{d, g} \rightarrow-\bar{J}_{d^{\prime}, g}$ induces a commutative diagram of rational maps (see, 
for example, [Uen75, II, Theorem 6.11])

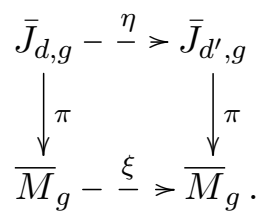

The rational map $\xi$ is the identity. Indeed, if $C \in M_{g}$ is very general and $C^{\prime}=\xi(C)$, then there is an induced birational map $J^{d} C \rightarrow J^{d^{\prime}} C^{\prime}$. As this is a birational map of abelian varieties, it is an isomorphism, and one concludes that $C \cong C^{\prime}$ using the Torelli theorem and the fact that for a very general curve, the Neron-Severi group of the Jacobian is isomorphic to $\mathbb{Z}$ (see [BFV12, Lemma 7.4] for more details). Having established that $\xi$ is the identity, the corollary follows from [Cap10, Proposition 3.2.2]. Again, we sketch the proof for the convenience of the reader. Let $U \subseteq M_{g}^{\circ}$ be an open set over which $\eta$ is defined. For each $C \in U$, there is an isomorphism $\left.\eta\right|_{C}: J^{d} C \rightarrow J^{d^{\prime}} C$. Since an isomorphism of abelian varieties is given by a translation followed by a group automorphism and $C$ is automorphism free, we have $\left.\eta\right|_{C}(L)=\left(L \otimes L_{C}\right)^{ \pm 1}$ for some $L_{C} \in$ $J^{ \pm\left(d^{\prime}-d\right)} C$ depending only on $C$ (see [Cap10, Lemma 3.2.3, Proposition 3.2.2] for more details). The assignment $C \mapsto L_{C}$ determines a rational section of $J_{ \pm\left(d^{\prime}-d\right), g} \rightarrow M_{g}$. The Franchetta conjecture (proven in [Mes87]) asserts that the only such sections are given by pluricanonical bundles.

Remark 8.15. It is likely that Corollary 8.14 fails for small values of $g$, where it is natural to expect that $J_{d, g}$ is rational for all values of $d \in \mathbb{Z}$.

\section{Appendix. Finite quotients of toric singularities}

The aim of this appendix is to study when a finite quotient of a toric singularity is Gorenstein, terminal, or canonical. We will work over an algebraically closed field $k$ of characteristic zero. The main focus is to generalize the Reid-Tai-Shepherd-Barron criterion for quotients of smooth varieties by finite groups. We expect that these type of results are well known to the experts, but we were unable to find a reference for the specific results we use, and so we include statements and proofs here.

\section{A.1 Finite quotient of smooth varieties}

Let us start by recalling the case of finite quotients of smooth varieties, which is well known and attributed to Khinich, Watanabe, Tai, Reid-Shepherd-Barron, and Reid (see, for example, [MS84, Theorem 2.3] and the references therein).

Theorem A.1. Let $G \subseteq \mathrm{GL}_{n}(k)$ be a finite subgroup, and assume that $G$ does not contain pseudo-reflections. Set $X=\mathbb{A}_{k}^{n} / G$. For each $g \in G$ of order $r \neq 1$ and each primitive $r$ th root of unity $\zeta$, write the eigenvalues of $g$ as $\zeta^{a_{1}}, \ldots, \zeta^{a_{n}}$ with $0 \leqslant a_{i}<r$ and define the age of $g$ with respect to $\zeta$ as

$$
\operatorname{age}(g, \zeta):=\frac{1}{r} \sum_{i=1}^{n} a_{i} .
$$

(i) (Khinich and Watanabe) The quotient $X$ is Gorenstein if and only if $G \subseteq \operatorname{SL}_{n}(k)$, that is,

$$
\operatorname{age}(g, \zeta) \in \mathbb{Z}
$$

for each $1 \neq g \in G$ and each (or, equivalently, some) primitive $r$ th root of unity $\zeta$. 


\section{S. Casalaina-Martin, J. L. Kass and F. Viviani}

(ii) (Reid-Shepherd-Barron [Rei87] and Tai [Tai82]) The quotient $X$ is canonical if and only if

$$
\operatorname{age}(g, \zeta) \geqslant 1
$$

for each $1 \neq g \in G$ and each primitive $r$ th root of unity $\zeta$.

(iii) (Reid [Rei87]) The quotient $X$ is terminal if and only if

$$
\operatorname{age}(g, \zeta)>1
$$

for each $1 \neq g \in G$ and each primitive $r$ th root of unity $\zeta$.

Remark A.2. Recall that an element $1 \neq g \in \mathrm{GL}_{n}(k)$ is a pseudo-reflection if its fixed locus $\operatorname{Fix}(g):=\left\{x \in \mathbb{A}_{k}^{n}: g \cdot x=x\right\}$ is a divisor inside $\mathbb{A}_{k}^{n}$. Equivalently, $1 \neq g \in \mathrm{GL}_{n}(k)$ is a pseudoreflection if and only if 1 is an eigenvalue of $g$ with multiplicity equal to $n-1$. In particular, if $1 \neq g \in \mathrm{GL}_{n}(k)$ is a pseudo-reflection, then $g \notin \mathrm{SL}_{n}(k)$. Note that:

(i) In Theorem A.1, if one removes the hypothesis that $G$ has no pseudo-reflections, the conditions (A.2) and (A.3) still imply canonical and terminal singularities, respectively.

(ii) If $G \subset \mathrm{GL}_{n}(k)$ is a finite group, denote by $G_{\mathrm{ps}}$ the normal subgroup of $G$ generated by the pseudo-reflections in $G$. Then $\mathbb{A}_{k}^{n} / G_{\mathrm{ps}}$ is smooth, that is, $\mathbb{A}_{k}^{n} / G_{\mathrm{ps}} \cong \mathbb{A}_{k}^{m}$ for some $m \leqslant n$, the quotient group $G / G_{\mathrm{ps}}$ acts linearly on $\mathbb{A}_{k}^{m}$ without pseudo-reflections, and $\mathbb{A}_{k}^{n} / G \cong \mathbb{A}_{k}^{m} /\left(G / G_{\mathrm{ps}}\right)$ (see $[$ Kol13, $\S 3.18]$ ). Therefore, we can always reduce to the case of finite groups acting without pseudo-reflections.

\section{A.2 Notation and background results on toric varieties}

We now recall some notation and background results on toric varieties, following [CLS11].

Fix a lattice $N$, that is, a free $\mathbb{Z}$-module of finite rank, and let $M=N^{\vee}$ be its dual lattice. Given a (convex, rational polyhedral) cone

$$
\sigma \subseteq N \otimes_{\mathbb{Z}} \mathbb{R}:=N_{\mathbb{R}},
$$

consider its dual cone (which is still convex, rational polyhedral)

$$
\sigma^{\vee}=\left\{\lambda \in M_{\mathbb{R}}:\langle\lambda, n\rangle \geqslant 0, \forall n \in \sigma\right\} \subset M \otimes_{\mathbb{Z}} \mathbb{R}=: M_{\mathbb{R}}
$$

The affine toric variety for the torus $\mathbb{T}:=\operatorname{Spec} k[M]=\mathbb{G}_{m} \otimes_{\mathbb{Z}} N$ associated with $\sigma \subset N_{\mathbb{R}}$ is given by

$$
U_{\sigma}=U_{\sigma, N}:=\operatorname{Spec} k\left[\sigma^{\vee} \cap M\right],
$$

where $k\left[\sigma^{\vee} \cap M\right]$ is the affine semigroup $k$-algebra associated with the normal affine semigroup $\sigma^{\vee} \cap M$ (by Gordon's lemma; see [CLS11, Proposition 1.2.17]). Note that the affine toric variety $U_{\sigma, N}$ depends both on the cone $\sigma \subset N_{\mathbb{R}}$ and on the lattice $N \subset N_{\mathbb{R}}$.

From here on, we will use the following notation:

- We denote by $\sigma(1)$ the set of one-dimensional faces of $\sigma$, that is, the extremal rays of the cone.

- Given $\rho \in \sigma(1)$, we set $u_{\rho}=u_{\rho, N}$ to be the primitive element of $\rho \cap N$. That is, $u_{\rho} \in \rho \cap N$ and if $u \in \rho \cap N$, then $u=n u_{\rho}$ for some $n \in \mathbb{N}$.

- We denote by $\Pi_{\sigma}=\Pi_{\sigma, N}$ the polytope $\Pi_{\sigma}=\operatorname{Conv}\left(0, u_{\rho, N}\right)_{\rho \in \sigma(1)}$, that is, the convex hull of 0 and the primitive elements of the extremal rays of $\sigma$, with respect to the lattice $N$.

Note that the primitive elements associated with the rays of $\sigma$ depend on the lattice $N$ we are considering; therefore, the polytope $\Pi_{\sigma, N}$ also depends upon the lattice $N$. 


\section{THE SINGULARITIES AND BIRATIONAL GEOMETRY}

In what follows, we will be using the following basic results on toric singularities.

Proposition A.3 (Gorenstein condition [CLS11, Propositions 8.2.12 and 11.4.11]). In the notation above, the affine toric variety $U_{\sigma}$ is Gorenstein if and only if there exists an $m_{\sigma} \in M$ such that

$$
\left\langle m_{\sigma}, u_{\rho}\right\rangle=1 \text { for all } \rho \in \sigma(1) \text {. }
$$

In this case, $U_{\sigma}$ has canonical singularities.

Proposition A.4 (QQQ-Gorenstein condition [CLS11, Proposition 11.4.12]). In the notation above, the following conditions are equivalent:

(i) The variety $U_{\sigma}$ is $\mathbb{Q}$-Gorenstein.

(ii) There exists an $m_{\sigma} \in M_{\mathbb{Q}}$ such that $\left\langle m_{\sigma}, u_{\rho}\right\rangle=1$ for all $\rho \in \sigma(1)$.

(iii) The polytope $\Pi_{\sigma}$ has a unique facet not containing the origin.

Note that the property of $U_{\sigma, N}=U_{\sigma}$ being $\mathbb{Q}$-Gorenstein depends both on the cone $\sigma$ and on the lattice $N$ (see Example A.10). This is not the case for the stronger property of $U_{\sigma, N}=U_{\sigma}$ being $\mathbb{Q}$-factorial, which is equivalent to the cone $\sigma$ being simplicial (see [CLS11, Theorem 11.4.8]) and hence depends only on the cone $\sigma$ and not on the lattice $N$.

Proposition A.5 (Canonical and terminal conditions [CLS11, Proposition 11.4.12]). In the notation above, assume that $U_{\sigma}$ is $\mathbb{Q}$-Gorenstein. Then $U_{\sigma}$ has canonical singularities if and only if the only non-zero lattice points in the polytope $\Pi_{\sigma}$ lie on the unique facet of $\Pi_{\sigma}$ not containing the origin. The variety $U_{\sigma}$ has terminal singularities if and only if the only lattice points of $\Pi_{\sigma}$ are its vertices.

\section{A.3 The case of cyclic groups}

In this subsection, we will consider the special case of a cyclic group $\mathbb{Z}_{r}:=\mathbb{Z} / r \mathbb{Z}$ acting on an affine toric variety $U_{\sigma}$, preserving the torus $\mathbb{T}=\operatorname{Spec} k[M]$.

After fixing a primitive $r$ th root of unity $\zeta \in k$, the action of $\mathbb{Z}_{r}$ on the coordinate ring $k[M]$ of $\mathbb{T}$ is given by a linear form $\lambda: M \rightarrow \mathbb{Z}$. It is well defined up to adding an $r$-multiple of a linear form. In other words, the action is uniquely determined by an element $[\lambda] \in N / r N=$ $\operatorname{Hom}_{\mathbb{Z}}(M, \mathbb{Z} / r \mathbb{Z})$. Explicitly, if we choose a primitive $r$ th root of unity $\zeta \in k$, we can identify the group $\mathbb{Z}_{r}$ with the subgroup of $k^{*}$ generated by $\zeta$ and the action on $k[M]$ is given by

$$
\zeta \cdot x^{m}=\zeta^{\lambda(m)} x^{m} .
$$

Moreover, if we fix an isomorphism $M \cong \mathbb{Z}^{n}$, so that $k[M]=k\left[x_{1}^{ \pm 1}, \ldots, x_{n}^{ \pm 1}\right]$, then the action of $\mathbb{Z}_{r}$ on $k[M]$ is given by

$$
\zeta \cdot x_{i}=\zeta^{a_{i}} x_{i} \quad \text { for some } 0 \leqslant a_{i}<r \quad(i=1, \ldots, n) .
$$

Proposition A.6. Let $N=\mathbb{Z}^{n}=\mathbb{Z}\left\langle e_{1}, \ldots, e_{n}\right\rangle$, and let $\sigma \subseteq N_{\mathbb{R}}$ be a (convex, rational polyhedral) cone. Let $\zeta$ be a primitive $r$ th root of unity, and suppose that $\mathbb{Z}_{r}=\langle\zeta\rangle$ acts on $U_{\sigma, N}$, preserving the torus $\mathbb{T}=\operatorname{Spec} k[M]$ and that the action on the ring $k[M]=k\left[x_{1}^{ \pm 1}, \ldots, x_{n}^{ \pm 1}\right]$ is given by

or, more explicitly, by

$$
\zeta \cdot x^{m}=\zeta^{\lambda(m)} x^{m} \quad \text { for some }[\lambda] \in N / r N
$$

$$
\zeta \cdot x_{i}=\zeta^{a_{i}} x_{i} \quad \text { for some } 0 \leqslant a_{i}<r \quad(i=1, \ldots, n) .
$$




\section{S. Casalaina-Martin, J. L. Kass and F. Viviani}

Then $U_{\sigma, N} / \mathbb{Z}_{r}$ is isomorphic to the affine toric variety $U_{\sigma, N^{\prime}}$, where $N^{\prime}$ is the super-lattice of $N$ given by

$$
N \subseteq N^{\prime}=N+\mathbb{Z}\left\langle\frac{1}{r} \lambda\right\rangle=\mathbb{Z}\left\langle e_{1}, \ldots, e_{n}, \sum_{i=1}^{n} \frac{a_{i} e_{i}}{r}\right\rangle \subset N_{\mathbb{Q}} .
$$

In particular, $U_{\sigma, N} / \mathbb{Z}_{r}$ is

(i) $\mathbb{Q}$-Gorenstein if and only if $\Pi_{\sigma, N^{\prime}}$ has a unique facet not containing the origin,

(ii) canonical if and only if $\Pi_{\sigma, N^{\prime}}$ has a unique facet not containing the origin and the only non-zero lattice points in $\Pi_{\sigma, N^{\prime}}$ lie in this facet,

(iii) terminal if and only if $\Pi_{\sigma, N^{\prime}}$ has a unique facet not containing the origin and the only lattice points of $\Pi_{\sigma, N^{\prime}}$ are its vertices.

Proof. Let $M^{\prime} \subseteq M$ be the sub-lattice of invariants; that is, $k\left[M^{\prime}\right]=k\left[x_{1}^{ \pm 1}, \ldots, x_{n}^{ \pm 1}\right]^{\mathbb{Z}_{r}}$. Clearly, the quotient $U_{\sigma, N} / \mathbb{Z}_{r}$ is the affine toric variety equal to Spec $k\left[\sigma \cap M^{\prime}\right]$. Therefore, in order to prove the first statement, we need to prove that after setting $N^{\prime}=N+\mathbb{Z}\left\langle\frac{1}{r} \lambda\right\rangle$, we have $\left(N^{\prime}\right)^{\vee}=M^{\prime} \subseteq M$. Since $N \subseteq N^{\prime}$, with torsion quotient, we have $M=N^{\vee} \supseteq\left(N^{\prime}\right)^{\vee}$. Now, pick an element $m=\sum_{i=1}^{n} m_{i} e_{i}^{\vee} \in M$ (with $m_{i} \in \mathbb{Z}$ ). Since $N^{\prime}$ is obtained from $N$ by adding the element $\frac{1}{r} \lambda=\sum_{i=1}^{n} a_{i} e_{i} / r \in N_{\mathbb{Q}}$, we have

$$
\begin{aligned}
m \in\left(N^{\prime}\right)^{\vee} & \Longleftrightarrow \sum_{i=1}^{n} \frac{a_{i} m_{i}}{r} \in \mathbb{Z} \Longleftrightarrow \sum_{i=1}^{n} a_{i} m_{i} \equiv 0 \quad(\bmod r) \\
& \Longleftrightarrow x^{m}:=\prod_{i=1}^{n} x_{i}^{m_{i}} \in k\left[x_{1}^{ \pm 1}, \ldots, x_{n}^{ \pm 1}\right]^{\mathbb{Z}_{r}} \Longleftrightarrow m \in M^{\prime} .
\end{aligned}
$$

The assertions (i)-(iii) now follow from this using Propositions A.4 and A.5.

Using Proposition A.6, we can prove the following criterion that plays a crucial role in the proof of Theorem 8.4.

Lemma A.7. For $i=1,2$, let $N_{i}$ be a lattice and let $\sigma_{i} \subset\left(N_{i}\right)_{\mathbb{R}}$ be a (convex, rational polyhedral) cone. Let $\phi: U_{\sigma_{1}, N_{1}} \rightarrow U_{\sigma_{2}, N_{2}}$ be a toric morphism induced by a homomorphism $\bar{\phi}: N_{1} \rightarrow N_{2}$ of lattices such that

(i) $\rho \in \sigma_{1}(1) \Rightarrow \bar{\phi}_{\mathbb{R}}(\rho) \in \sigma_{2}(1)$;

(ii) for every $\rho \in \sigma_{1}(1)$, we have $\bar{\phi}\left(u_{\rho, N_{1}}\right)=u_{\bar{\phi}_{\mathbb{R}}(\rho), N_{2}}$.

Now, suppose that the cyclic group $\mathbb{Z}_{r}$ acts on the $U_{\sigma_{i}, N_{i}}$, preserving the torus $\mathbb{T}_{i}=\mathbb{G}_{m} \otimes_{\mathbb{Z}} N_{i}$ for $i=1,2$ and assume that

(a) $\phi: U_{\sigma_{1}, N_{1}} \rightarrow U_{\sigma_{2}, N_{2}}$ is $\mathbb{Z}_{r}$-equivariant,

(b) $U_{\sigma_{2}, N_{2}}$ is smooth and $\mathbb{Z}_{r}$ acts on $U_{\sigma_{2}, N_{2}}$ without pseudo-reflections.

Then $U_{\sigma_{1}, N_{1}} / \mathbb{Z}_{r}$ is $\mathbb{Q}$-Gorenstein. Moreover, if $U_{\sigma_{2}, N_{2}} / \mathbb{Z}_{r}$ has canonical singularities, then $U_{\sigma_{1}, N_{1}}$ has canonical singularities.

Proof. Using the above notation, fix a primitive $r$ th root of unity $\zeta$, and suppose that the action of $\mathbb{Z}_{r}$ on $U_{\sigma_{i}, N_{i}}$ is determined by the element $\left[\lambda_{i}\right] \in N_{i} / r N_{i}$. Since $\phi$ is $\mathbb{Z}_{r}$-equivariant by condition (a), we must have $\bar{\phi}\left(\left[\lambda_{1}\right]\right)=\left[\lambda_{2}\right]$, so that the homomorphism $\bar{\phi}: N_{1} \rightarrow N_{2}$ extends to a homomorphism (which we will still denote by $\bar{\phi}$ )

$$
\bar{\phi}: \quad N_{1}^{\prime}:=N_{1}+\mathbb{Z}\left\langle\frac{1}{r} \lambda_{1}\right\rangle \longrightarrow N_{2}^{\prime}:=N_{2}+\mathbb{Z}\left\langle\frac{1}{r} \lambda_{2}\right\rangle .
$$


By Proposition A.6, the toric morphism $\widetilde{\phi}: U_{\sigma_{1}, N_{1}^{\prime}} \rightarrow U_{\sigma_{2}, N_{2}^{\prime}}$ induced by $\bar{\phi}$ coincides with the quotient map $U_{\sigma_{1}, N_{1}} / \mathbb{Z}_{r} \rightarrow U_{\sigma_{2}, N_{2}} / \mathbb{Z}_{r}$ induced by $\phi$.

Now, fix an extremal ray $\rho$ of $\sigma_{1}$ and look at $\bar{\phi}_{\mathbb{R}}(\rho)$, which is an extremal ray of $\sigma_{2}$ by property (i). Since $N_{1} \subseteq N_{1}^{\prime}$, the two primitive elements along the ray $\rho$ with respect to the above lattices are related by $u_{\rho, N_{1}}=c \cdot u_{\rho, N_{1}^{\prime}}$ for some $c \in \mathbb{Z}_{>0}$. On the other hand, it follows from condition (b) that $u_{\bar{\phi}_{\mathbb{R}}(\rho), N_{2}}=u_{\bar{\phi}_{\mathbb{R}}(\rho), N_{2}^{\prime}}$. Moreover, it follows from property (ii) that $\bar{\phi}\left(u_{\rho, N_{1}}\right)=$ $u_{\bar{\phi}_{\mathbb{R}}(\rho), N_{2}}$. Finally, we will have $\bar{\phi}\left(u_{\rho, N_{1}^{\prime}}\right)=l \cdot u_{\bar{\phi}_{\mathbb{R}}(\rho), N_{2}^{\prime}}$ for some $l \in \mathbb{Z}_{>0}$. Combining everything, we find that

$$
u_{\bar{\phi}_{\mathbb{R}}(\rho), N_{2}^{\prime}}=u_{\bar{\phi}_{\mathbb{R}}(\rho), N_{2}}=\bar{\phi}\left(u_{\rho, N_{1}}\right)=c \cdot \bar{\phi}\left(u_{\rho, N_{1}^{\prime}}\right)=c \cdot l \cdot u_{\bar{\phi}_{\mathbb{R}}(\rho), N_{2}^{\prime}},
$$

from which we deduce that $c=l=1$, in other words, that

$$
u_{\rho, N_{1}}=u_{\rho, N_{1}^{\prime}} \quad \text { and } \quad \bar{\phi}\left(u_{\rho, N_{1}^{\prime}}\right)=u_{\bar{\phi}_{\mathbb{R}}(\rho), N_{2}^{\prime}} .
$$

Now, observe that since $U_{\sigma_{2}, N_{2}}$ is smooth by condition (b), the quotient $U_{\sigma_{2}, N_{2}} / \mathbb{Z}_{r}=U_{\sigma_{2}, N_{2}^{\prime}}$ is $\mathbb{Q}$-factorial, hence in particular $\mathbb{Q}$-Gorenstein. By Proposition A.4, there exists an $m_{2} \in\left(M_{2}^{\prime}\right)_{\mathbb{Q}}=$ $\left(M_{2}\right)_{\mathbb{Q}}=\left(N_{2}^{\vee}\right)_{\mathbb{Q}}$ such that $\left\langle m_{2}, u_{\tau, N_{2}^{\prime}}\right\rangle=1$ for every extremal ray $\tau$ of $\sigma_{2}$. From (A.6), we see that the element $m_{1}=\left(\bar{\phi}_{\mathbb{R}}\right)^{\vee}\left(m_{2}\right) \in\left(M_{1}^{\prime}\right)_{\mathbb{Q}}=\left(M_{1}\right)_{\mathbb{Q}}=\left(N_{1}^{\vee}\right)_{\mathbb{Q}}$ satisfies (for every extremal ray $\rho$ of $\left.\sigma_{1}\right)$

$$
\left\langle m_{1}, u_{\rho, N_{1}^{\prime}}\right\rangle=\left\langle\left(\bar{\phi}_{\mathbb{R}}\right)^{\vee}\left(m_{2}\right), u_{\rho, N_{1}^{\prime}}\right\rangle=\left\langle m_{2}, \bar{\phi}\left(u_{\rho, N_{1}^{\prime}}\right)\right\rangle=\left\langle m_{2}, u_{\bar{\phi}_{\mathbb{R}}(\rho), N_{2}^{\prime}}\right\rangle=1,
$$

which shows that $U_{\sigma_{1}, N_{1}^{\prime}}=U_{\sigma_{1}, N_{1}} / \mathbb{Z}_{r}$ is $\mathbb{Q}$-Gorenstein.

Now, take a point $0 \neq x \in N_{1}^{\prime}$ which belongs to $\Pi_{\sigma_{1}, N_{1}^{\prime}}$, that is,

$$
x=\sum_{\rho \in \sigma_{1}(1)} \alpha_{\rho} \cdot u_{\rho, N_{1}^{\prime}} \text { with } \alpha_{\rho} \geqslant 0 \text { and } 0<\sum_{\rho \in \sigma_{1}(1)} \alpha_{\rho} \leqslant 1 .
$$

Using (A.6), we get

$$
\bar{\phi}(x)=\sum_{\rho \in \sigma_{1}(1)} \alpha_{\rho} \cdot u_{\bar{\phi}_{\mathbb{R}}(\rho), N_{2}^{\prime}} \Rightarrow 0 \neq \bar{\phi}(x) \in \Pi_{\sigma_{2}, N_{2}^{\prime}} .
$$

If $U_{\sigma_{2}, N_{2}^{\prime}}=U_{\sigma_{2}, N_{2}} / \mathbb{Z}_{r}$ has canonical singularities, then Proposition A.5 implies that $\bar{\phi}(x)$ belongs to the unique facet of $\Pi_{\sigma_{2}, N_{2}^{\prime}}$ not containing the origin. This is equivalent to the fact that $\sum_{\rho \in \sigma_{1}(1)} \alpha_{\rho}=1$, which then implies that $x$ also belongs to the unique facet of $\Pi_{\sigma_{1}, N_{1}^{\prime}}$ not containing the origin, in other words, that $U_{\sigma_{1}, N_{1}^{\prime}}=U_{\sigma_{1}, N_{1}} / \mathbb{Z}_{r}$ has canonical singularities.

Although we will not use this, just for the sake of completeness, we prove the following criterion for a cyclic quotient of an affine Gorenstein toric variety to be Gorenstein.

Proposition A.8. We use the same notation as in Proposition A.6. Assume furthermore that $U_{\sigma, N}$ is Gorenstein, so that there is an $m_{\sigma} \in M$ such that

$$
\left\langle m_{\sigma}, u_{\rho}\right\rangle=1 \text { for all } \rho \in \sigma(1),
$$

where $u_{\rho}$ is the primitive element along the ray $\rho$ with respect to the lattice $N$. If $\lambda$ and $m_{\sigma}$ satisfy $\frac{1}{r} \lambda\left(m_{\sigma}\right) \in \mathbb{Z}$, then $U_{\sigma, N} / \mathbb{Z}_{r}$ is Gorenstein.

Proof. We will use the notation of the proof of Proposition A.6. The assumption $\frac{1}{r} \lambda\left(m_{\sigma}\right) \in \mathbb{Z}$ implies that $m_{\sigma} \in M^{\prime}=\left(N^{\prime}\right)^{\vee}$. Moreover, the fact that $\left\langle m_{\sigma}, u_{\rho}\right\rangle=1$ ensures that $u_{\rho}$ is still a 


\section{S. Casalaina-Martin, J. L. Kass and F. Viviani}

primitive generator of $\rho \in \sigma(1)$ with respect to $N^{\prime}$ : indeed, if $u_{\rho}=l \cdot \tilde{u}_{\rho}$ for some $2 \leqslant l \in \mathbb{N}$ and $\tilde{u}_{\rho} \in N^{\prime}$, then

$$
1=\left\langle m_{\sigma}, u_{\rho}\right\rangle=l\left\langle m_{\sigma}, \tilde{u}_{\rho}\right\rangle \Rightarrow\left\langle m_{\sigma}, \tilde{u}_{\rho}\right\rangle \notin \mathbb{Z},
$$

which contradicts the fact that $m_{\sigma} \in\left(N^{\prime}\right)^{\vee}$.

Remark A.9. If we apply Propositions A.6 and A.8 to the case $U_{\sigma, N}=\mathbb{A}_{k}^{n}$, we get back one direction of Theorem A.1 for finite cyclic quotients of smooth varieties.

We warn the reader that, contrary to the fact that finite quotients of $\mathbb{Q}$-factorial toric singularities are $\mathbb{Q}$-factorial (because the factoriality of $U_{\sigma, N}$ is equivalent to the fact that the cone $\sigma$ is simplicial), a finite quotient of a Gorenstein toric singularity need not to be $\mathbb{Q}$-Gorenstein, as the following example shows.

Example A.10. Let $N=\mathbb{Z}^{3}=\mathbb{Z}\left\langle e_{1}, e_{2}, e_{3}\right\rangle$, and consider the toric variety $U_{\sigma, N}$ defined by the cone

$$
\sigma=\mathbb{R}_{\geqslant 0}\left\langle e_{1}, e_{2}, e_{3}, e_{1}+e_{2}-e_{3}\right\rangle \subseteq \mathbb{R}^{3}=N \otimes \mathbb{R} .
$$

Now, let $\mathbb{Z}_{2}$ act by -1 on $x_{1}$ and by 1 on $x_{2}$ and $x_{3}$. One can easily check using Propositions A.3 and A.4 that $U_{\sigma, N}$ is Gorenstein, while $U_{\sigma, N} / \mathbb{Z}_{2}$ is not $\mathbb{Q}$-Gorenstein.

\section{A.4 Reduction to the cyclic case}

In this subsection, we show that in order to detect if a finite quotient $V / G$ of a normal $k$-variety has canonical or terminal singularities, it is enough to check only that the cyclic quotients $V / C$ are canonical or terminal as $C$ varies among all the cyclic subgroups of $G$. The result in the case where $V$ is smooth appears in a number of places (for example [HM82, Appendix 1 to $\S 1$, proof of Reid-Tai's criterion], [Kol13, Theorem 3.21]). The argument for singular $V$ is the same, and while we expect that the result is well known in this case as well, we are unaware of a reference, and so we include the proof here for the convenience of the reader.

Theorem A.11. Suppose that $G$ is a finite group acting on $V$, a normal scheme of finite type over $k$. Then $V / G$ has canonical (respectively, terminal) singularities if and only if for every cyclic subgroup $C \leqslant G$, the quotient $V / C$ has canonical (respectively, terminal) singularities.

Proof. We will follow the proof of [Kol13, Theorem 3.21], which deals with the case $V=\mathbb{A}_{k}^{n}$. First, suppose that $X=V / G$ does not have canonical (respectively, terminal) singularities. Let $\widetilde{X} \rightarrow X$ be a resolution of singularities, and let $E \subseteq \widetilde{X}$ be a prime divisor such that the discrepancy satisfies $a(E, X)<0$ (respectively, $a(E, X) \leqslant 0$ ). Let $p: \widetilde{V} \rightarrow \widetilde{X}$ be the normalization of $\widetilde{X}$ in the field of fractions of $V$, and let $F \subseteq \widetilde{V}$ be a prime divisor dominating $E$. We have a commutative diagram

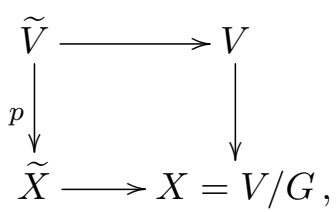

where the vertical morphisms are finite and the horizontal ones are birational. It is computed in $[$ Kol13, (2.42.4)] that the discrepancies of $F$ and $E$ are related by the formula

$$
a(E, X)+1=\frac{a(F, V)+1}{\left|C_{F}\right|} .
$$


The group $G$ acts on the field of fractions of $V$, and one can easily check that the action preserves integrality, so $G$ also acts on $\widetilde{V}$ and $\widetilde{X}=\widetilde{V} / G$. Let $C_{F}$ be the subgroup of $G$ acting as the identity on $F$. Since $\widetilde{V}$ is generically smooth along $F$, the subgroup $C_{F} \leqslant G$ is cyclic. The diagram (A.7) factors as follows:

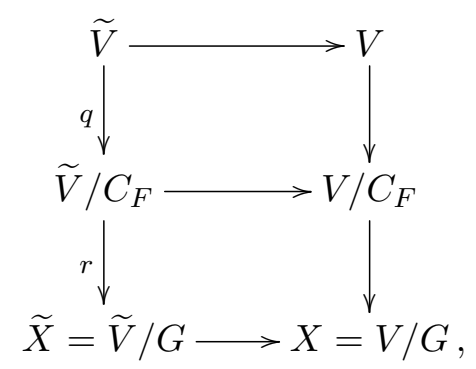

where again the vertical morphisms are finite and the horizontal ones are birational. Consider the prime divisor $E^{\prime}=q(F)$, which is exceptional over $V / C_{F}$. By applying formula (A.8) to the morphism $q$, we get

$$
a\left(E^{\prime}, V / C_{F}\right)+1=\frac{a(F, V)+1}{\left|C_{F}\right|},
$$

which, together with (A.8), implies that $a\left(E^{\prime}, V / C_{F}\right)=a(E, X)<0$ (respectively, $\left.a(E, X) \leqslant 0\right)$. Consequently, we see that $V / C_{F}$ does not have canonical (respectively, terminal) singularities.

Conversely, suppose there is a cyclic group $C \leqslant G$ such that $V / C$ does not have canonical (respectively, terminal) singularities. Let $(V / C)^{\sim} \rightarrow V / C$ be a resolution of singularities, and suppose that $E^{\prime}$ is an exceptional divisor such that $a\left(E^{\prime}, V / C\right)<0$ (respectively, $\left.a\left(E^{\prime}, V / C\right) \leqslant 0\right)$. Let $\widetilde{V}$ be the integral closure of $(V / C)^{\sim}$ in the field of fractions of $V$, and let $F \subseteq \widetilde{V}$ be a prime divisor dominating $E^{\prime}$. Again, we obtain (A.10). Now, by a result of Zariski and Abhyankar [Kol13, Lemma 2.22] there is a diagram

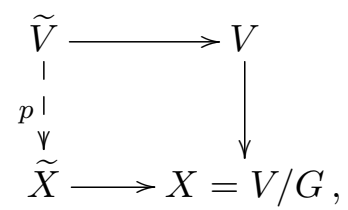

where the bottom morphism is birational, $p$ is the induced rational map, and $F$ dominates a prime divisor $E$ of $\widetilde{X}$. The computation of [Kol13, (2.42.4)] holds (see especially the discussion at the end of the proof of [Kol13, Corollary 2.43)]), giving (A.8). Thus we have $a(E, X)=a\left(E^{\prime}, V / C\right)<0$ (respectively, $a(E, X) \leqslant 0$ ), and it follows that $X$ does not have canonical (respectively, terminal) singularities.

\section{ACKNOWLEDGEMENTS}

The first author would like to thank Jonathan Wise for conversations on toric geometry and deformation theory and James McKernan for a discussion on singularities of toric varieties.

\section{REFERENCES}

BFV12 G. Bini, C. Fontanari, and F. Viviani, On the birational geometry of the universal Picard variety, Int. Math. Res. Not. 2012 (2012), no. 4, 740-780; https://doi.org/10.1093/imrn/rnr045. 


\section{S. Casalaina-Martin, J. L. Kass and F. Viviani}

BH98 W. Bruns and J. Herzog, Cohen-Macaulay rings, Cambridge Stud. Adv. Math., vol. 39 (Cambridge Univ. Press, Cambridge, 1998); https://doi.org/10.1017/CB09780511608681.

Cap94 L. Caporaso, A compactification of the universal Picard variety over the moduli space of stable curves, J. Amer. Math. Soc. 7 (1994), no. 3, 589-660; https://doi.org/10.2307/2152786.

Cap10 , Compactified Jacobians of nodal curves, Notes for a mini-course given at the Istituto Superiore Tecnico of Lisbon, February 1-4, 2010, available at http://www.mat.uniroma3.it/ users/caporaso/cjac.pdf.

CKV13 S. Casalaina-Martin, J. L. Kass, and F. Viviani, The geometry and combinatorics of cographic toric face rings, Algebra Number Theory 7 (2013), no. 8, 1781-1815; https://doi.org/10. 2140/ant.2013.7.1781.

CKV15 _ The local structure of compactified Jacobians, Proc. Lond. Math. Soc. (3) 110 (2015), no. 2, 510-542; https://doi.org/10.1112/plms/pdu063.

CLS11 D. A. Cox, J. B. Little, and H. K. Schenck, Toric varieties, Grad. Stud. Math., vol. 124 (Amer. Math. Soc., Providence, RI, 2011); https://doi.org/10.1090/gsm/124.

Cot12 E. Cotterill, Effective divisors on $\overline{\mathscr{M}}_{g}$ associated to curves with exceptional secant planes, Manuscripta Math. 138 (2012), no. 1-2, 171-202; https://doi.org/10.1007/ s00229-011-0491-4.

EH87 D. Eisenbud and J. Harris, The Kodaira dimension of the moduli space of curves of genus $\geqslant 23$, Invent. Math. 90 (1987), no. 2, 359-387; https://doi.org/10.1007/BF01388710.

Far09 G. Farkas, Birational aspects of the geometry of $\overline{\mathscr{M}}_{g}$, in Surveys in Differential Geometry, Vol. XIV, Geometry of Riemann Surfaces and their Moduli Spaces, Surv. Differ. Geom., vol. 14 (Int. Press, Somerville, MA, 2009), 57-110; https://doi.org/10.4310/SDG.2009.v14.n1.a3.

Far10 The birational type of the moduli space of even spin curves, Adv. Math. 223 (2010), no. 2, 433-443; https://doi.org/10.1016/j.aim.2009.08.011.

Far12 Theta characteristics and their moduli, Milan J. Math. 80 (2012), no. 1, 1-24; https: //doi.org/10.1007/s00032-012-0178-7.

Fon05 C. Fontanari, On the geometry of moduli of curves and line bundles, Atti Accad. Naz. Lincei Cl. Sci. Fis. Mat. Natur. Rend. Lincei (9) Mat. Appl. 16 (2005), no. 1, 45-59.

FP05 G. Farkas and M. Popa, Effective divisors on $\overline{\mathscr{M}}_{g}$, curves on $K 3$ surfaces, and the slope conjecture, J. Algebraic Geom. 14 (2005), no. 2, 241-267; https://doi.org/10.1090/ S1056-3911-04-00392-3.

FV12 G. Farkas and A. Verra, Moduli of theta-characteristics via Nikulin surfaces, Math. Ann. 354 (2012), no. 2, 465-496; https://doi.org/10.1007/s00208-011-0739-z.

FV13 , The classification of universal Jacobians over the moduli space of curves, Comment. Math. Helv. 88 (2013), no. 3, 587-611; https://doi.org/10.4171/CMH/297.

FV14 The geometry of the moduli space of odd spin curves, Ann. of Math. 180 (2014), no. 3, 927-970; https://doi.org/10.4007/annals.2014.180.3.3.

GKZ94 I. M. Gel'fand, M. M. Kapranov, and A. V. Zelevinsky, Discriminants, resultants, and multidimensional determinants, in Mathematics: Theory $\&$ Applications (Birkhäuser Boston, Inc., Boston, MA, 1994); https://doi.org/10.1007/978-0-8176-4771-1.

HM82 J. Harris and D. Mumford, On the Kodaira dimension of the moduli space of curves, Invent. Math. 67 (1982), no. 1, 23-88; https://doi.org/10.1007/BF01393371.

Kaw85 Y. Kawamata, Minimal models and the Kodaira dimension of algebraic fiber spaces, J. reine angew. Math. 363 (1985), 1-46; https://doi.org/10.1515/crll.1985.363.1.

Kol13 J. Kollár, Singularities of the minimal model program, Cambridge Tracts in Math., vol. 200 (Cambridge Univ. Press, Cambridge, 2013); https://doi.org/10.1017/CB09781139547895.

Lud07 K. Ludwig, Moduli of spin curves, Ph.D. Thesis, University of Hannover, 2007, available at http://edok01.tib.uni-hannover.de/edoks/e01dh07/530657929.pdf. 


\section{THE SINGULARITIES AND BIRATIONAL GEOMETRY}

Mat02 K. Matsuki, Introduction to the Mori program, Universitext S(pringer-Verlag, New York, 2002); https://doi.org/10.1007/978-1-4757-5602-9.

Mes87 N. Mestrano, Conjecture de Franchetta forte, Invent. Math. 87 (1987), no. 2, 365-376; https: //doi.org/10.1007/BF01389421.

MS84 D. R. Morrison and G. Stevens, Terminal quotient singularities in dimensions three and four, Proc. Amer. Math. Soc. 90 (1984), no. 1, 15-20; https://doi.org/10.2307/2044659.

Muk96 S. Mukai, Curves and K3 surfaces of genus eleven, Moduli of Vector Bundles (Sanda, 1994; Kyoto, 1994), Lecture Notes in Pure and Appl. Math., vol. 179 (Dekker, New York, 1996), 189197.

Pan96 R. Pandharipande, A compactification over $\bar{M}_{g}$ of the universal moduli space of slope-semistable vector bundles, J. Amer. Math. Soc. 9 (1996), no. 2, 425-471; https://doi.org/10.1090/ S0894-0347-96-00173-7.

Pri67 D. Prill, Local classification of quotients of complex manifolds by discontinuous groups, Duke Math. J. 34 (1967), 375-386; https://doi.org/10.1215/S0012-7094-67-03441-2.

Rei87 M. Reid, Young person's guide to canonical singularities, Algebraic Geometry, Bowdoin, 1985 (Brunswick, Maine, 1985), Proc. Sympos. Pure Math., vol. 46 (Amer. Math. Soc., Providence, RI, 1987), 345-414.

Rim80 D. S. Rim, Equivariant G-structure on versal deformations, Trans. Amer. Math. Soc. 257 (1980), no. 1, 217-226; https://doi.org/10.2307/1998132.

Ser80 J.-P. Serre, Trees (Springer-Verlag, Berlin - New York, 1980); https://doi.org/10.1007/ 978-3-642-61856-7.

Tai82 Y.-S. Tai, On the Kodaira dimension of the moduli space of abelian varieties, Invent. Math. 68 (1982), no. 3, 425-439; https://doi.org/10.1007/BF01389411.

Tan98 S.-L. Tan, On the slopes of the moduli spaces of curves, Internat. J. Math. 9 (1998), no. 1, 119-127; https://doi.org/10.1142/S0129167X98000087.

Uen75 K. Ueno, Classification theory of algebraic varieties and compact complex spaces, Lecture Notes in Math., vol. 439 (Springer-Verlag, Berlin - New York, 1975); https://doi.org/10.1007/ $\mathrm{BFb} 0070570$.

Ver05 A. Verra, The unirationality of the moduli spaces of curves of genus 14 or lower, Compos. Math. 141 (2005), no. 6, 1425-1444; https://doi.org/10.1112/S0010437X05001685.

Sebastian Casalaina-Martin casa@math.colorado.edu

University of Colorado, Department of Mathematics, Campus Box 395, Boulder, CO 80309, USA

Jesse Leo Kass kassj@math.sc.edu

Department of Mathematics, University of South Carolina, 1523 Greene Street, Columbia, SC 29208, USA

Filippo Viviani viviani@mat.uniroma3.it

Dipartimento di Matematica e Fisica, Università Roma Tre, Largo S. Leonardo Murialdo 1, 00146 Roma, Italy 